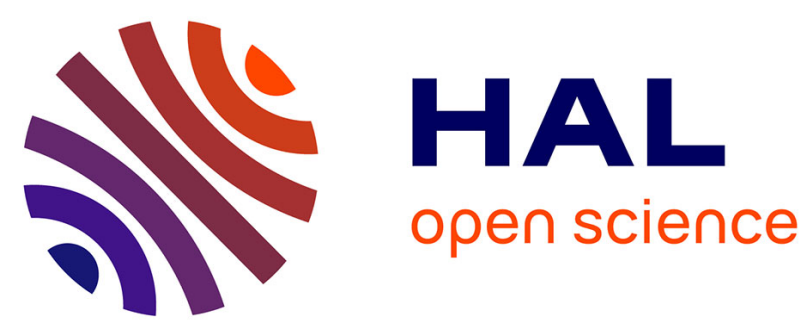

\title{
Tuning the solid-state emission of small push-pull dipolar dyes to the far-red through variation of the electron-acceptor group
}

Sébastien Redon, Gwenaelle Eucat, Martin Ipuy, Erwann Jeanneau, Isabelle Gautier-Luneau, Alain Ibanez, Chantal Andraud, Yann Bretonnière

\section{To cite this version:}

Sébastien Redon, Gwenaelle Eucat, Martin Ipuy, Erwann Jeanneau, Isabelle Gautier-Luneau, et al.. Tuning the solid-state emission of small push-pull dipolar dyes to the far-red through variation of the electron-acceptor group. Dyes and Pigments, 2018, 156, pp.116-132. 10.1016/j.dyepig.2018.03.049 . hal-01856313

\section{HAL Id: hal-01856313 \\ https://hal-amu.archives-ouvertes.fr/hal-01856313}

Submitted on 10 Aug 2018

HAL is a multi-disciplinary open access archive for the deposit and dissemination of scientific research documents, whether they are published or not. The documents may come from teaching and research institutions in France or abroad, or from public or private research centers.
L'archive ouverte pluridisciplinaire HAL, est destinée au dépôt et à la diffusion de documents scientifiques de niveau recherche, publiés ou non, émanant des établissements d'enseignement et de recherche français ou étrangers, des laboratoires publics ou privés. 


\title{
Tuning the Solid-State Emission of Small Push-pull Dipolar Dyes to the Far-red Through Variation of the Electron-acceptor Group
}

\author{
Sébastien Redon, ${ }^{a}$ Gwenaëlle Eucat,,${ }^{a, b}$ Martin Ipuy, ${ }^{a}$ Erwann Jeanneau, ${ }^{c}$ Isabelle Gautier-Luneau, ${ }^{b}$ \\ Alain Ibanez, ${ }^{b}$ Chantal Andraud ${ }^{*, a}$ and Yann Bretonnière ${ }^{*, a}$ \\ ${ }^{a}$ Univ Lyon, ENS de Lyon, CNRS UMR 5182, Université Lyon 1, Laboratoire de Chimie, F-69342 \\ Lyon (France). \\ ${ }^{b}$ Univ. Grenoble Alpes, Institut Néel, F-38042 Grenoble (France). \\ CNRS, Institut Néel, F-38042 Grenoble (France). \\ Institute of Engineering, Univ. Grenoble Alpes \\ ${ }^{c}$ Centre de Diffractométrie Henri Longchambon, Université Lyon I, 43 boulevard du 11 Novembre \\ 1918, F-69622 Villeurbanne Cedex (France). \\ chantal.andraud@ens-lyon.fr/yann.bretonniere@ens-lyon.fr
}

\begin{abstract}
Series of solid-state emitters based on the D- $\pi$-A dipolar structure and featuring various electron-donor and electron-acceptor groups were designed, and their spectroscopic properties studied. From weak emission in dilute solutions, intense emissions in aggregated state (AIE) and in the crystalline state were obtained. Analysis in light of crystal structures obtained by X-ray diffraction revealed specific crystal packing and presence of long chain of emitting aggregates. This simple molecular engineering around the D- $\pi$-A dipolar structure provides easy access to a wide range of effective solid-state emitters allowing modulation of emission wavelengths up to the near infrared ( $\lambda_{\mathrm{em}}$ reaching 735 and $768 \mathrm{~nm}$ for compound $\mathbf{2 f}$ and $\mathbf{3 f}$ bearing the strongest electron-withdrawing group).
\end{abstract}

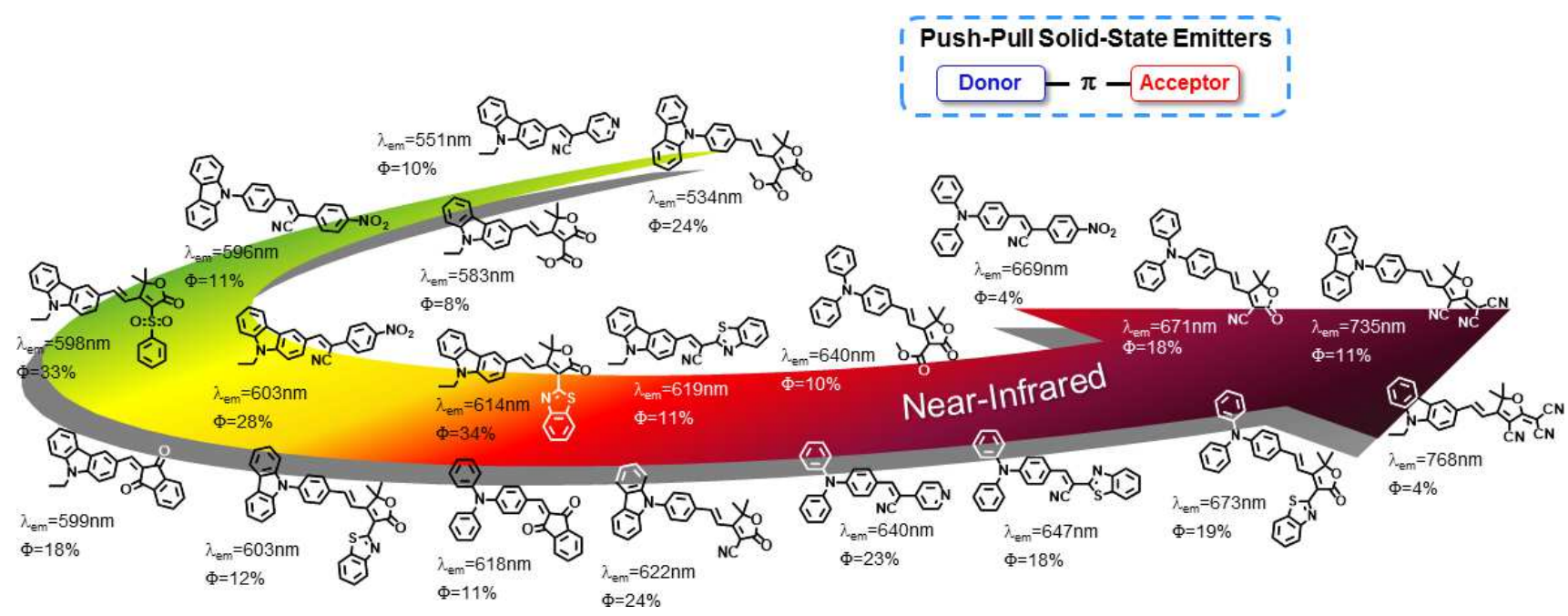

Highlights

- Library of small push-pull dipolar solid-state emitters has been obtained featuring three different electron-donor groups and various electro-acceptor groups.

- New electron-acceptor groups based on substituted 2(5H)-furanone rings are presented. 
- Fluorescence properties in the aggregated state and in the solid-state are described, and correlated to the presence of specific aggregates in the crystal structure.

- The best dyes displayed near-infrared emission in the solid-state with emission quantum yield of $11 \%$ at $\lambda_{\mathrm{em}}=735 \mathrm{~nm}$ and $4 \%$ at $\lambda_{\mathrm{em}}=768 \mathrm{~nm}$.

\section{Keywords}

Solid-state fluorescence; Push-pull dyes; Organic dyes; Near-infrared; Aggregation-induced emission; $2(5 \mathrm{H})$-furanone rings

\section{Introduction}

Engineering around organic solid-state emitters has become a thriving area of research these last few years, impelled by potential applications in the field of optoelectronics, materials and bio-imaging.[1-4] In that particular context, fluorescent organic nanocrystals or emissive organic nanoparticles based on fluorophores displaying aggregation-induced emission (AIE) properties are a promising alternative to dissolved fluorophores or to inorganic quantum dots.[5, 6] Advantages include higher absorption and higher photo-stability, combined to the infinite possibilities offered by organic synthesis to functionalize the dye and to tune the spectroscopic properties. In particular, shifting the emission wavelength towards the far-red and even the near infrared (NIR) in the first biological transparency window $\left(\lambda_{\mathrm{em}}>650 \mathrm{~nm}\right)$ is a requisite for deeper in-vivo imaging. [7-10] This wavelength range allows enhanced penetration depth and better contrast compared to the visible, due to lower absorption, minimized scattering and lower self-fluorescence from biological samples. However so far, good solid-state fluorophores with far-red / NIR emissions are still scarce despite increasing number of structures available and a tremendous work on AIE-active fluorophore design.[5, 11] On the one hand, interesting long wavelength solid-state emitters can be obtained by decorating red emitting dyes with the AIE-active segment, classically triphenylethene,[12-16] but also triphenylamine which also acts as potent electron-donor group.[17-21] However, this molecular engineering gives rise to rather big molecular weights and complex structures requiring sophisticated syntheses. On the other hand, recent works highlighted the interest of simple and small molecular weight $D-\pi-A$ push-pull dipolar fluorophores, in which an electron donating group $D$ is connected to an electron-withdrawing group $A$ via a $\pi$-conjugated bridge, for the design of efficient solid-state emitters.[22-27]'[28-31]'[32-39]'[40]'[41]

The permanent dipole moment associated with the $D-\pi$-A structure gives rise to strong dipole-dipole interactions that can induce specific organization and orientation of molecules in the solid-state and favors the formation of emissive aggregates. Moreover, since the fluorescence of such dipolar fluorophores is usually characterized by a large Stokes shift, red and even far-red emission wavelengths over $700 \mathrm{~nm}$ are accessible, providing appropriate combinations of electron-donor and acceptor groups. Association of electron-deficient $2 \mathrm{H}$-indazoles with electron-rich heteroarenes [42] or of quinolinemalononitrile electron-withdrawing group with the electron-rich triphenylamino group [43] gives access to low molecular weight fluorophores with emission wavelengths exceeding $720 \mathrm{~nm}$ in the solid-state, as recently reported. Tuning of the substituents on the core skeleton also greatly influence the molecular organization in the crystal state and consequently the emission quantum yield in the solid-state, as this was illustrated in our earlier work on methoxy-substituted push-pull fluorophores bearing 2dicyanomethylene-3-cyano-4,5,5-trimethyl-2,5-dihydrofurane (TCF) as electron-acceptor group.[41] Varying the number and the position of the methoxy groups have a drastic impact on the solid-state emission modulating the emission wavelength from $580 \mathrm{~nm}$ to $730 \mathrm{~nm}$ for the reddest shifted dye, while also influencing the emission efficiency. Similarly, with 2-(3,5,5-trimethylcyclohex-2-en-1ylidene)malononitrile (dicyanoisophorone) as electron-accepting group and $N, N$-dialkylamino group as electron-donor, emission above $700 \mathrm{~nm}$ could be obtained when $J$-aggregates in the form of inclined alignment of dipoles are present in the packing resulting in sharpening of the excitation and red-shift of fluorescence.[40]

In continuation of our previous works on dipolar solid-state emitters, [40-41] we want to present here a 
library of push-pull fluorophores featuring 4-( $N, N$-diphenylamino)phenyl- (Series 1, Chart 1), 9-ethyl9H-carbazolyl- (Series 2) and 4-(9H-carbazol-9-yl)- (Series 3) as electron-donor groups and various electron-acceptor groups (A - J, Scheme 1), in particular 2(5H)-furanone rings with a weak electronwithdrawing group at the $\mathrm{C} 2$ position $(\mathbf{G}-\mathbf{J})$. If some of these acceptor groups such as indanedione (A), dicyanoisophorone $(\mathbf{E})$ or $T C F(\mathbf{F})$ are quite commonly used in several high efficiency solid-state emitters, [32, 38-41, 44-50] other 2(5H)-furanones have rarely been utilized as acceptors entities in dye design and only within the frame of non-linear optical chromophores design (acceptor group G).[51, 52]
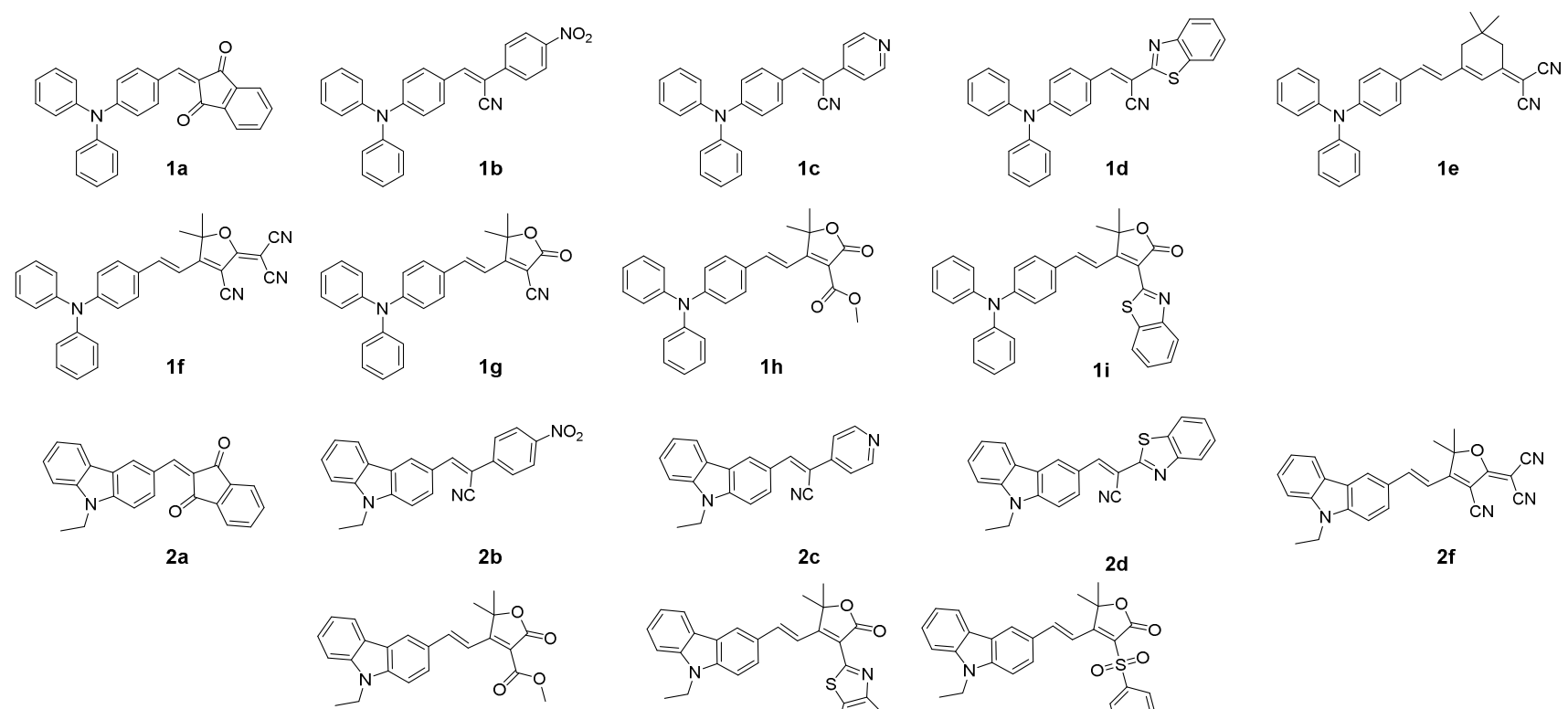

$2 \mathrm{~h}$
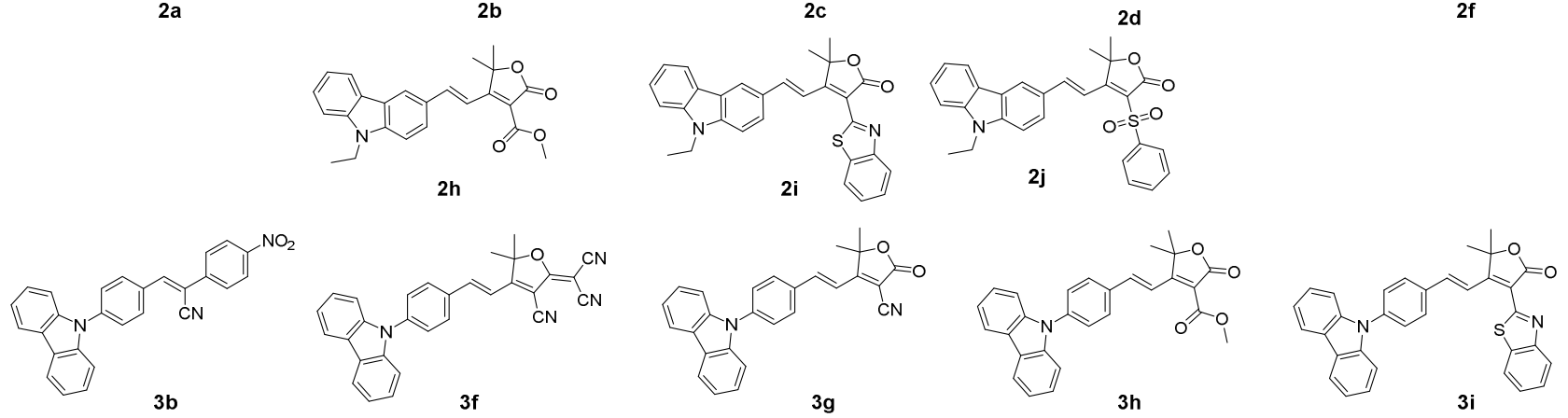

Chart 1. Structures of fluorophores $1 \mathbf{a}-\mathbf{- 1 i}, \mathbf{2 a}-\mathbf{2} \mathbf{j}$ and $\mathbf{3 b}-\mathbf{3 i}$.

\section{Experimental}

\subsection{General Information}

Commercially available materials and reagent grade solvents were used as received. Microwave syntheses were conducted in $20 \mathrm{~mL}$ sealed tube on a Biotage Initiator 2.5 single-mode reactor using external IR temperature control. The reaction monitoring was performed by analytical thin-layer chromatography (TLC) on Merck 60 F254 precoated silica gel plate $(0.2 \mathrm{~mm}$ thickness) with visualization using a UV lamp. Purification by column chromatography was carried out using 35-70 am silica gel. ${ }^{1} \mathrm{H}$ and ${ }^{13} \mathrm{C}$ NMR spectra were recorded at ambient temperature on a Bruker Advance 300, 400 or 500 operating at $300.1,400.0$ or $500.0 \mathrm{MHz}$ for ${ }^{1} \mathrm{H}$ and $75.0,101.00$ or $125.0 \mathrm{MHz}$ for ${ }^{13} \mathrm{C}$, respectively. Chemical shifts are reported as $\delta$ values (ppm) with reference to the residual solvent peaks. For proton, data are reported as follows: chemical shift, multiplicity $(\mathrm{s}=\operatorname{singlet}, \mathrm{d}=$ doublet, $\mathrm{t}=$ triplet, $\mathrm{q}=$ quartet, $\mathrm{m}=$ multiplet, $\mathrm{b}=$ broad), coupling constants in Hz. IR spectra were recorded on a FT/IR4200 type A spectrometer. High-resolution mass spectrometry measurements were performed at the Centre Commun de Spectrométrie de Masse (UCBL, Villeurbanne, France). Melting points were recorded on a calibrated Koffler bench. Ethyl phenylsulfonylacetate,[53] compounds 1, E and 1e,[40] 3,[54] and F [41] were obtained according to reported procedures. Spectroscopic data for known compounds 1a,[55] 1b,[56] 1c,[57] 2a,[39] 2b,[56] 2c,[58] 2f [59] matched literature values.

\subsection{Synthetic procedures and characterization data}


2.2.1 General Protocol 1: Knoevenagel Reaction. Aldehyde (1 equiv.) and acceptor compound (1 equiv.) were dissolved in acetonitrile (100 $\mathrm{mL}$ for $14 \mathrm{mmol}$.). Piperidine (0.01 equiv.) was added and solution was stirred at the temperature indicated for the time indicated in each protocol. The solution was then concentrated under vacuum and the product purified by column chromatography on silica gel.

2.2.2 General Protocol 2: Microwave Reaction. Aldehyde (1 equiv.) and acceptor compound (1 equiv.) were dissolved in a solution of dried acetonitrile $(1 \mathrm{~mL}$ for $1 \mathrm{mmol})$ in a microwave tube and 2 drops of piperidine were added. The tube was sealed and the mixture was heated at $110^{\circ} \mathrm{C}$ for 40 minutes by focused microwave irradiation under controlled temperature. After cooling to room temperature, the solvents were evaporated under vacuum and the product was purified by column chromatography on silica gel.

2.2.3 4,5,5'-trimethyl-2-oxo-2,5-dihydrofuran-3-carbonitrile (G). Sodium (80 mg, $3.3 \mathrm{mmol})$ was slowly added at $0^{\circ} \mathrm{C}$ to dry methanol $(4 \mathrm{~mL})$. Once everything was dissolved, 3-hydroxy-3-methylbutan-2-one $4(650 \mu \mathrm{L}, 6.1 \mathrm{mmol})$ and ethyl cyanoacetate $(1.4 \mathrm{~mL}, 13 \mathrm{mmol})$ were added at $0^{\circ} \mathrm{C}$. After $12 \mathrm{~h}$, the reaction was quenched by the addition of acetic acid $(5 \mathrm{~mL})$. The solvents were evaporated under reduced pressure. The crude was partitioned between water $(25 \mathrm{~mL})$ and $\mathrm{CH}_{2} \mathrm{Cl}_{2}(25 \mathrm{~mL})$. The organic phase was dried on $\mathrm{Na}_{2} \mathrm{SO}_{4}$, filtered and evaporated. Purification by column chromatography on silica gel eluting with ethyl acetate / cyclohexane (30/70, v/v) gave $\mathbf{G}$ as yellow solid $(1.25 \mathrm{~g}$, yield = 82\%): m.p. $54^{\circ} \mathrm{C}$; ${ }^{1} \mathrm{H}-\mathrm{NMR}\left(\mathrm{CDCl}_{3}, 300 \mathrm{MHz}, \mathrm{a} / \mathrm{ppm}\right) 1.50(\mathrm{~s}, 6 \mathrm{H}), 2.28(\mathrm{~s}, 3 \mathrm{H}) ;{ }^{13} \mathrm{C}-\mathrm{NMR}\left(\mathrm{CDCl}_{3}\right.$, $101 \mathrm{MHz}, \mathrm{a} / \mathrm{ppm}) 184.8,165.4,110.7,104.5,88.3,24.3,13.8$; IR $\left(\mathrm{v} / \mathrm{cm}^{-1}\right) 3448,2239,1764,1650$, $1288,1080,910$.

2.2.4 Methyl 4,5,5'-trimethyl-2-oxo-2,5-dihydrofuran-3-carboxylate (H). Sodium (80 mg, $3.3 \mathrm{mmol}$ ) was added slowly at $0^{\circ} \mathrm{C}$ to dry methanol $(4 \mathrm{~mL})$. After full dissolution, 3-hydroxy-3-methylbutan-2-one $4(650 \mu \mathrm{L}, 6.1 \mathrm{mmol})$ and methyl malonate $(700 \mu \mathrm{L}, 6.1 \mathrm{mmol})$ were added at $0^{\circ} \mathrm{C}$. After $2 \mathrm{~h}$, the reaction was quenched by adding a saturated aqueous $\mathrm{NH}_{4} \mathrm{Cl}$ solution $(10 \mathrm{~mL})$. EtOAc $(25 \mathrm{~mL})$ was then added. The organic phase was separated, dried on $\mathrm{Na}_{2} \mathrm{SO}_{4}$, filtered and concentrated under reduced pressure. Purification by column chromatography on silica gel eluting with EtOAc/petroleum ether $(30 / 70, \mathrm{v} / \mathrm{v})$ afforded $\mathbf{H}(710 \mathrm{mg}$, yield $=59 \%)$ as white solid; m.p. $65^{\circ} \mathrm{C} ;{ }^{1} \mathrm{H}-\mathrm{NMR}\left(\mathrm{CDCl}_{3}\right.$, $300 \mathrm{MHz}, \mathrm{a} / \mathrm{ppm}) 3.88$ (s, 3H), 2.35 (s, 3H), 1.48 (s, 6H); ${ }^{13} \mathrm{C}-\mathrm{NMR}\left(\mathrm{CDCl}_{3}, 101 \mathrm{MHz}, \mathrm{a} / \mathrm{ppm}\right)$ 181.2, 167.2, 162.2, 118.0, 85.4, 52.1, 24.3, 13.0; IR $\left(\mathrm{v} / \mathrm{cm}^{-1}\right)$ 1760, 1711, 1350, 1280, 1191, 1174, 1043, 969, 808.

2.2.5 3-(benzo[d]thiazol-2-yl)-4,5,5-trimethylfuran-2(5H)-one (I). G (906 mg, $6 \mathrm{mmol}), 2$ hydroxythiophenol $(640 \mu \mathrm{L}, 6 \mathrm{mmol})$ and $85 \%$ aqueous phosphoric acid $(3 \mathrm{~g})$ were heated at $120^{\circ} \mathrm{C}$ for $14 \mathrm{~h}$. After cooling down to room temperature, water $(50 \mathrm{~mL})$ was added. The mixture was carefully neutralized by slow addition of saturated aqueous $\mathrm{K}_{2} \mathrm{CO}_{3}(50 \mathrm{~mL})$. The solution was extracted with dichloromethane three times. The combined organic layers were washed with water, dried over $\mathrm{Na}_{2} \mathrm{SO}_{4}$, filtered and evaporated to dryness to give $\mathbf{I}$, used without further purification. Brown solid (1.41 g, yield $=91 \%)$; m.p. $158-160^{\circ} \mathrm{C} ;{ }^{1} \mathrm{H}-\mathrm{NMR}\left(\mathrm{CDCl}_{3}, 300 \mathrm{MHz}, \mathrm{a} / \mathrm{ppm}\right) 8.07(\mathrm{~d}, J=8.2 \mathrm{~Hz}, 1 \mathrm{H}), 7.94(\mathrm{~d}, J=$ $8.2 \mathrm{~Hz}, 1 \mathrm{H}), 7.53(\mathrm{t}, J=8.2 \mathrm{~Hz}, 1 \mathrm{H}), 7.43(\mathrm{t}, J=8.2 \mathrm{~Hz}, 1 \mathrm{H}), 2.68(\mathrm{~s}, 3 \mathrm{H}), 1.59(\mathrm{~s}, 6 \mathrm{H}) ;{ }^{13} \mathrm{C}-\mathrm{NMR}$ $\left(\mathrm{CDCl}_{3}, 101 \mathrm{MHz}, \mathrm{a} / \mathrm{ppm}\right) 172.3,170.3,157.9,153.0,135.1,126.3,125.6,123.3,121.8,119.0,87.0$,

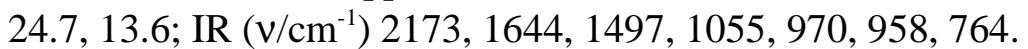

2.2.6 4,5,5'-trimethyl-3-(phenylsulfonyl)furan-2(5H)-one (J). Sodium (70 mg, $3.04 \mathrm{mmol})$ was slowly added at $0^{\circ} \mathrm{C}$ to dry methanol $(5 \mathrm{~mL})$. After complete dissolution, 3-hydroxy-3-methylbutan-2-one $4(1.05 \mathrm{~mL}, 10 \mathrm{mmol}$.) and ethyl phenylsulfonylacetate $(2.51 \mathrm{~g}, 11 \mathrm{mmol})$ were added at $0^{\circ} \mathrm{C}$. The mixture was then heated at $50^{\circ} \mathrm{C}$ overnight. After cooling to room temperature, the solvent was removed under reduced atmosphere to give an oily residue. $\mathrm{Et}_{2} \mathrm{O}$ was added to precipitate the product. The solid was filtered, washed with $\mathrm{Et}_{2} \mathrm{O}$ and dried. Recrystallization in a mixture $\mathrm{Et}_{2} \mathrm{O} / \mathrm{EtOAc}$ $(40 \mathrm{~mL} / 4 \mathrm{~mL})$ gave compound $\mathbf{J}$ as white solid $(1.5 \mathrm{~g}, 56 \%)$; m.p. $172{ }^{\circ} \mathrm{C} ;{ }^{1} \mathrm{H}-\mathrm{NMR}\left(\mathrm{CDCl}_{3}, 300 \mathrm{MHz}\right.$, 口/ppm) $8.08(\mathrm{~d}, J=7.5 \mathrm{~Hz}, 2 \mathrm{H}), 7.66(\mathrm{t}, J=7.3 \mathrm{~Hz}, 1 \mathrm{H}), 7.56(\mathrm{t}, J=7.2 \mathrm{~Hz}, 2 \mathrm{H}), 2.51(\mathrm{~s}, 3 \mathrm{H}), 1.47$ (s, $6 \mathrm{H}) .{ }^{13} \mathrm{C}-\mathrm{NMR}\left(\mathrm{CDCl}_{3}, 101 \mathrm{MHz}, \mathrm{a} / \mathrm{ppm}\right) 179.1,164.7,139.4,134.4,129.3,128.8,126.9,86.7,24.3$, 12.6. IR $\left(\mathrm{v} / \mathrm{cm}^{-1}\right) 1746,1320,1311,1266,1149,1084,1029,719,685$. 
2.2.7 2-(4-(diphenylamino)benzylidene)-1H-indene-1,3(2H)-dione (1a). From 1 (400 mg, $1.45 \mathrm{mmol}$.) and $\mathbf{A}\left(255 \mathrm{mg}, 1.75 \mathrm{mmol}\right.$.) following general protocol $1\left(40^{\circ} \mathrm{C}, 18 \mathrm{~h}\right)$. Purification by chromatography (Petroleum ether, EtOAc: $9 / 1 \mathrm{v} / \mathrm{v}$ ) afforded the title compound 1a. Red solid (230 mg, yield $=33 \%)$; m.p. $214^{\circ} \mathrm{C} ;{ }^{1} \mathrm{H}-\mathrm{NMR}\left(\mathrm{CD}_{2} \mathrm{Cl}_{2}, 400 \mathrm{MHz}, \delta / \mathrm{ppm}\right) 8.41(\mathrm{~d}, J=8.8 \mathrm{~Hz}, 2 \mathrm{H}), 8.06-7.85$ $(\mathrm{m}, 2 \mathrm{H}), 7.76(\mathrm{dd}, J=10.7,5.3 \mathrm{~Hz}, 3 \mathrm{H}), 7.39(\mathrm{t}, J=7.7 \mathrm{~Hz}, 4 \mathrm{H}), 7.23(\mathrm{~d}, J=6.2 \mathrm{~Hz}, 6 \mathrm{H}), 6.99(\mathrm{~d}, J=$ $8.8 \mathrm{~Hz}, 2 \mathrm{H}) ;{ }^{13} \mathrm{C}-\mathrm{NMR}\left(\mathrm{CD}_{2} \mathrm{Cl}_{2}, 101 \mathrm{MHz}, \delta / \mathrm{ppm}\right) 191.3(\mathrm{CO}), 153.2,146.5(\mathrm{CH}), 146.2,142.9,140.5$, $137.2(2 \times \mathrm{CH}), 135.3(\mathrm{CH}), 135.12(\mathrm{CH}), 130.3(4 \times \mathrm{CH}), 127.2(4 \times \mathrm{CH}), 126.1(2 \times \mathrm{CH}), 123.2(\mathrm{CH})$, $123.1(\mathrm{CH}), 119.1(2 \times \mathrm{CH})$; IR $\left(\mathrm{v} / \mathrm{cm}^{-1}\right)$ 3062, $1680(\mathrm{Vco}), 1570 / 1542 / 1487,1265,1154$; HRMS (ESI $\left.{ }^{+}\right)$ calcd for $\mathrm{C}_{28} \mathrm{H}_{20} \mathrm{NO}_{2}[\mathrm{M}+\mathrm{H}]^{+}: 402.1489$, found: 402.1482; UV-Vis $\left(\mathrm{CH}_{2} \mathrm{Cl}_{2}\right) \lambda_{\max }=485 \mathrm{~nm}(\varepsilon=$ $\left.37800 \mathrm{~L} \cdot \mathrm{mol}^{-1} \cdot \mathrm{cm}^{-1}\right)$.

2.2.8 (Z)-3-(4-(diphenylamino)phenyl)-2-(4-nitrophenyl)acrylonitrile $\quad(1 \mathrm{~b}) . \quad$ From 1 (350 mg, $1.25 \mathrm{mmol}$.) and $\mathbf{B}\left(250 \mathrm{mg}, 1.5 \mathrm{mmol}\right.$.) following general protocol $1\left(45^{\circ} \mathrm{C}, 4 \mathrm{~h}\right)$. Purification by chromatography $\left(\mathrm{CH}_{2} \mathrm{Cl}_{2}\right)$ afforded the title compound 1b. Red solid (340 mg, yield = 33\%); m.p. $145^{\circ} \mathrm{C} ;{ }^{1} \mathrm{H}-\mathrm{NMR}\left(\mathrm{CD}_{2} \mathrm{Cl}_{2}, 500 \mathrm{MHz}, \delta / \mathrm{ppm}\right) 8.27(\mathrm{~d}, J=8.8 \mathrm{~Hz}, 2 \mathrm{H}), 7.83(\mathrm{dd}, J=12.6,8.9 \mathrm{~Hz}, 4 \mathrm{H})$, $7.60(\mathrm{~s}, 1 \mathrm{H}), 7.36(\mathrm{t}, J=7.8 \mathrm{~Hz}, 4 \mathrm{H}), 7.19(\mathrm{~d}, J=7.9 \mathrm{~Hz}, 6 \mathrm{H}), 7.03(\mathrm{~d}, J=8.8 \mathrm{~Hz}, 2 \mathrm{H}) ;{ }^{13} \mathrm{C}$ NMR $(125$ $\left.\mathrm{MHz}, \mathrm{CD}_{2} \mathrm{Cl}_{2}, \delta / \mathrm{ppm}\right) 151.8,148.1,147.0,145.5,142.3,132.2(2 \times \mathrm{CH}), 130.4(4 \mathrm{x} \mathrm{CH}), 126.9(4 \mathrm{x}$ $\mathrm{CH}), 126.9(2 \times \mathrm{CH}), 126.0,125.7(2 \times \mathrm{CH}), 125.0(2 \times \mathrm{CH}), 120.6(2 \times \mathrm{CH}), 118.8,105.5(\mathrm{CN})$; IR $\left(\mathrm{v} / \mathrm{cm}^{-1}\right)$ 3060, $2208(\mathrm{VCN}), 1572 / 1490 / 1508,1338,1198,700$; HRMS (ESI ${ }^{+}$) calcd for $\mathrm{C}_{27} \mathrm{H}_{20} \mathrm{~N}_{3} \mathrm{O}_{2}$ $[\mathrm{M}+\mathrm{H}]^{+}: 418.1550$, found: $418.1543 ; \mathrm{UV}-\mathrm{Vis}\left(\mathrm{CH}_{2} \mathrm{Cl}_{2}\right) \lambda_{\max }=456 \mathrm{~nm}\left(\varepsilon=28700 \mathrm{~L}_{\mathrm{mol}}{ }^{-1} \cdot \mathrm{cm}^{-1}\right)$.

2.2.9 (Z)-3-(4-(diphenylamino)phenyl)-2-(pyridin-4-yl)acrylonitrile (1c). From 1 (100 mg, $0.4 \mathrm{mmol}$.) and $\mathbf{C}\left(70 \mathrm{mg}, 0.45 \mathrm{mmol}\right.$.) following general protocol $1\left(40^{\circ} \mathrm{C}, 4 \mathrm{~h}\right)$. Purification by chromatography $\left(\mathrm{CH}_{2} \mathrm{Cl}_{2} / \mathrm{Et}_{3} \mathrm{~N}\right.$ : $95 / 5$ then $\left.92 / 8 \mathrm{v} / \mathrm{v}\right)$ afforded the title compound 1c. Orange solid $(90 \mathrm{mg}$, yield $=60 \%)$; m.p. $182^{\circ} \mathrm{C} .{ }^{1} \mathrm{H}-\mathrm{NMR}\left(\mathrm{CD}_{2} \mathrm{Cl}_{2}, 500 \mathrm{MHz}, \delta / \mathrm{ppm}\right) 8.62(\mathrm{~d}, J=4.8 \mathrm{~Hz}, 2 \mathrm{H}), 7.83$ $(\mathrm{d}, J=8.8 \mathrm{~Hz}, 4 \mathrm{H}), 7.64(\mathrm{~s}, 1 \mathrm{H}), 7.56-7.46(\mathrm{~m}, 2 \mathrm{H}), 7.35(\mathrm{t}, J=7.8 \mathrm{~Hz}, 4 \mathrm{H}), 7.18(\mathrm{t}, J=8.0 \mathrm{~Hz}, 6 \mathrm{H})$, $7.03(\mathrm{~d}, J=8.8 \mathrm{~Hz}, 2 \mathrm{H}) ;{ }^{13} \mathrm{C}-\mathrm{NMR}\left(\mathrm{CD}_{2} \mathrm{Cl}_{2}, 125 \mathrm{MHz}, \delta / \mathrm{ppm}\right) 151.1,150.6(2 \mathrm{x} \mathrm{CH}), 146.4,144.4$, 142.5, $131.5(2 \times \mathrm{CH}), 129.8(4 \times \mathrm{CH}), 126.3(4 \times \mathrm{CH}), 125.3,125.0(2 \times \mathrm{CH}), 120.0(2 \times \mathrm{CH}), 119.6(2$ x CH), 117.9, 104.6 (CN); IR (v/cm $\left.{ }^{-1}\right)$ 3057, 2924, $2206\left(v_{\mathrm{CN}}\right), 1577 / 1557 / 1487,1176,756 . \mathrm{HRMS}$ $\left(\mathrm{ESI}^{+}\right)$calcd for $\mathrm{C}_{26} \mathrm{H}_{20} \mathrm{~N}_{3}[\mathrm{M}+\mathrm{H}]^{+}: 374.1652$, found: 374.1656 ; UV-Vis $\left(\mathrm{CH}_{2} \mathrm{Cl}_{2}\right) \lambda_{\max }=427 \mathrm{~nm}(\varepsilon=$ $\left.29700 \mathrm{~L} \cdot \mathrm{mol}^{-1} \cdot \mathrm{cm}^{-1}\right)$.

2.2.10 (E)-2-(benzo[d]thiazol-2-yl)-3-(4-(diphenylamino)phenyl)acrylonitrile (1d). From 1 (700 mg, $2.5 \mathrm{mmol}$.) and $\mathbf{D}\left(520 \mathrm{mg}, 3 \mathrm{mmol}\right.$.) following general protocol 1 ( $\left.40^{\circ} \mathrm{C}, 4 \mathrm{~h}\right)$. Purification by chromatography (eluting with $\mathrm{CH}_{2} \mathrm{Cl}_{2}$ ) afforded the title compound $\mathbf{1 d}$. Red solid $(840 \mathrm{mg}$, yield = $79 \%)$; m.p. $173{ }^{\circ} \mathrm{C} ;{ }^{1} \mathrm{H}-\mathrm{NMR}\left(\mathrm{CD}_{2} \mathrm{Cl}_{2}, 500 \mathrm{MHz}, \delta / \mathrm{ppm}\right) 8.05(\mathrm{~s}, 1 \mathrm{H}), 8.01(\mathrm{~d}, J=5.0 \mathrm{~Hz}, 1 \mathrm{H}), 7.92-$ $7.89(\mathrm{~m}, 3 \mathrm{H}), 7.52(\mathrm{dd}, J=11.2,4.0 \mathrm{~Hz}, 1 \mathrm{H}), 7.41(\mathrm{dd}, J=11.2,4.0 \mathrm{~Hz}, 1 \mathrm{H}), 7.39-7.30(\mathrm{~m}, 4 \mathrm{H}), 7.26$ - $7.14(\mathrm{~m}, 6 \mathrm{H}), 7.02(\mathrm{~d}, J=8.9 \mathrm{~Hz}, 2 \mathrm{H}) ;{ }^{13} \mathrm{C}-\mathrm{NMR}\left(\mathrm{CD}_{2} \mathrm{Cl}_{2}, 125 \mathrm{MHz}, \delta / \mathrm{ppm}\right) 164.9,154.5,152.4$, $147.2(\mathrm{CH}), 146.7(\mathrm{CH}), 135.4,132.9(2 \times \mathrm{CH}), 130.5(4 \times \mathrm{CH}), 127.5(\mathrm{CH}), 127.1(4 \times \mathrm{CH}), 126.3$ $(\mathrm{CH}), 126.0(2 \times \mathrm{CH}), 125.2,123.8(\mathrm{CH}), 122.4(\mathrm{CH}), 120.2(2 \mathrm{x} \mathrm{CH}), 118.0,101.8(\mathrm{CN}) ; \mathrm{IR}\left(\mathrm{v} / \mathrm{cm}^{-1}\right)$ 3056, $2210\left(\mathrm{~V}_{\mathrm{CN}}\right), 1570 / 1487 / 1506,1430,1174,987$; HRMS $\left(\mathrm{ESI}^{+}\right)$calcd for $\mathrm{C}_{28} \mathrm{H}_{20} \mathrm{~N}_{3} \mathrm{~S}[\mathrm{M}+\mathrm{H}]^{+}$: 430.1372, found: 430.1357; UV-Vis $\left(\mathrm{CH}_{2} \mathrm{Cl}_{2}\right) \lambda_{\max }=454 \mathrm{~nm}\left(\varepsilon=31200 \mathrm{~L} \cdot \mathrm{mol}^{-1} \cdot \mathrm{cm}^{-1}\right)$.

\subsubsection{1 (E)-2-[3-cyano-4-(4-(diphenylamino)styryl)-5,5-dimethylfuran-2(5H)-}

ylidene]malononitrile (1f). From 1 (546 mg, $2 \mathrm{mmol}$.) and TCF F (318 mg, $1.6 \mathrm{mmol}$.) following a slightly modified general protocol 1 . Toluene $(2 \mathrm{~mL})$ was added in the initial mixture. The mixture was heated at $80^{\circ} \mathrm{C}$ for $24 \mathrm{~h}$, then cooled to room temperature. The mixture was concentrated under vacuum and $\mathrm{Et}_{2} \mathrm{O}(10 \mathrm{~mL})$ was added to precipitate the compound. It was then filtered, washed with $\mathrm{Et}_{2} \mathrm{O}(2 \times 10$ $\mathrm{mL})$ and with ethanol $(2 \times 10 \mathrm{~mL})$, and dried. 1f was obtained as green solid (380 mg, yield = 53\%); m.p. $>250^{\circ} \mathrm{C}(\mathrm{dec}) ;{ }^{1} \mathrm{H}$ NMR $\left(500 \mathrm{MHz}, \mathrm{CDCl}_{3}, \delta / \mathrm{ppm}\right) 7.58(\mathrm{~d}, J=16.1 \mathrm{~Hz}, 1 \mathrm{H}), 7.46(\mathrm{~d}, J=8.4 \mathrm{~Hz}, 2 \mathrm{H})$, $7.36(\mathrm{t}, J=7.1 \mathrm{~Hz}, 4 \mathrm{H}), 7.20(\mathrm{dd}, J=18.9,7.4 \mathrm{~Hz}, 6 \mathrm{H}), 6.98(\mathrm{~d}, J=8.4 \mathrm{~Hz}, 2 \mathrm{H}), 6.82(\mathrm{~d}, J=16.1 \mathrm{~Hz}$, $1 \mathrm{H}), 1.76(\mathrm{~s}, 6 \mathrm{H}) ;{ }^{13} \mathrm{C} \mathrm{NMR}\left(125 \mathrm{MHz}, \mathrm{CDCl}_{3}, \delta / \mathrm{ppm}\right)$ 176.2, 174.3, 152.9, $147.6(\mathrm{CH}), 146.0,131.5(2$ x CH), $130.2(4 \times \mathrm{CH}), 126.7(4 \times \mathrm{CH}), 126.3,126.0(2 \times \mathrm{CH}), 120.3(2 \times \mathrm{CH}), 112.6,111.8,111.6$ $(\mathrm{CH}), 111.3,97.5(2 \times \mathrm{CN}), 97.1(\mathrm{CN}), 56.6,27.0\left(2 \times \mathrm{CH}_{3}\right)$; IR $\left(\mathrm{v} / \mathrm{cm}^{-1}\right) 2223\left(\mathrm{v}_{\mathrm{CN}}\right), 1742,1560,1545$, 
1528, 1488, 1330, 1283, 1266, 1168, 758, 698; HRMS (ESI ${ }^{+}$calcd for $\mathrm{C}_{30} \mathrm{H}_{23} \mathrm{~N}_{4} \mathrm{O}[\mathrm{M}+\mathrm{H}]^{+}: 455.1866$, found: 455.1857; UV-Vis $\left(\mathrm{CH}_{2} \mathrm{Cl}_{2}\right) \lambda_{\max }=565 \mathrm{~nm}\left(\varepsilon=42000 \mathrm{~L} \cdot \mathrm{mol}^{-1} \cdot \mathrm{cm}^{-1}\right)$.

2.2.12 3-cyano-4-[2-[4-(diphenylamino)phenyl]ethenyl]-5,5-dimethyl-2-butenolide (1g). From $1(273 \mathrm{mg}, 1 \mathrm{mmol})$ and $\mathbf{G}(151 \mathrm{mg}, 1 \mathrm{mmol})$ in acetonitrile $(2 \mathrm{~mL})$ and toluene $(0.5 \mathrm{~mL}) \mathrm{using}$ general protocol 2. Purification by chromatography (EtOAc/Petroleum ether: 30/70) afforded the title compound 1g. Red solid (340 mg, yield = 83\%); m.p. $171{ }^{\circ} \mathrm{C} ;{ }^{1} \mathrm{H}-\mathrm{NMR}\left(\mathrm{CD}_{2} \mathrm{Cl}_{2}, 500 \mathrm{MHz}, \delta / \mathrm{ppm}\right) 7.65$ $(\mathrm{d}, J=16.3 \mathrm{~Hz}, 1 \mathrm{H}), 7.49(\mathrm{~d}, J=8.8 \mathrm{~Hz}, 2 \mathrm{H}), 7.35(\mathrm{t}, J=7.9 \mathrm{~Hz}, 4 \mathrm{H}), 7.17(\mathrm{t}, J=7.8 \mathrm{~Hz}, 6 \mathrm{H}), 6.99(\mathrm{~d}$, $J=8.8 \mathrm{~Hz}, 2 \mathrm{H}), 6.75(\mathrm{~d}, J=16.3 \mathrm{~Hz}, 1 \mathrm{H}), 1.65(\mathrm{~s}, 6 \mathrm{H}) ;{ }^{13} \mathrm{C}-\mathrm{NMR}\left(\mathrm{CD}_{2} \mathrm{Cl}_{2}, 125 \mathrm{MHz}, \delta / \mathrm{ppm}\right) 177.1$ (CO), 167.2, 151.9, 146.8, $146.0(\mathrm{CH}), 130.6(2 \times \mathrm{CH}), 130.2(4 \times \mathrm{CH}), 127.0,126.6(4 \times \mathrm{CH}), 125.5(2$ x CH), $120.8(2 \times \mathrm{CH}), 113.3,112.3(\mathrm{CH}), 96.7(\mathrm{CN}), 87.4,26.4\left(2 \times \mathrm{CH}_{3}\right) ; \mathrm{IR}\left(\mathrm{v} / \mathrm{cm}^{-1}\right) 2223\left(\mathrm{~V}_{\mathrm{CN}}\right)$, 1735 ( $\left.v_{\mathrm{co}}\right), 1556,1506,1486,1279,1262,1175,697$; HRMS (ESI ${ }^{+}$) calcd for $\mathrm{C}_{27} \mathrm{H}_{22} \mathrm{~N}_{2} \mathrm{O}_{2} \mathrm{Na}[\mathrm{M}+\mathrm{Na}]^{+}$: 429.7573, found: 429.1570; UV-Vis $\left(\mathrm{CH}_{2} \mathrm{Cl}_{2}\right) \lambda_{\max }=484 \mathrm{~nm}\left(\varepsilon=38400 \mathrm{~L} \cdot \mathrm{mol}^{-1} \cdot \mathrm{cm}^{-1}\right)$.

2.2.13 3-methyl ester-4-[2-[4-(diphenylamino)phenyl]ethenyl]-5,5-dimethyl-2-butenolide (1h). From 1 ( $273 \mathrm{mg}, 1 \mathrm{mmol}$.) and $\mathbf{H}\left(200 \mathrm{mg}, 1 \mathrm{mmol}\right.$.) following general protocol $1\left(80^{\circ} \mathrm{C}, 24 \mathrm{~h}\right)$. Purification by chromatography (EtOAc/Pentane: 20/80) afforded the title compound $\mathbf{1 h}$. Yellow solid $(350 \mathrm{mg}$, yield $=74 \%)$; m.p. $174{ }^{\circ} \mathrm{C} ;{ }^{1} \mathrm{H}-\mathrm{NMR}\left(\mathrm{CDCl}_{3}, 500 \mathrm{MHz}, \delta / \mathrm{ppm}\right) 7.75(\mathrm{~d}, J=16.9 \mathrm{~Hz}, 1 \mathrm{H})$, $7.46(\mathrm{~d}, J=8.7 \mathrm{~Hz}, 2 \mathrm{H}), 7.36-7.26(\mathrm{~m}, 4 \mathrm{H}), 7.18(\mathrm{~d}, J=16.9 \mathrm{~Hz}, 1 \mathrm{H}), 7.15-7.11(\mathrm{~m}, 6 \mathrm{H}), 7.01(\mathrm{~d}, J$ $=8.7 \mathrm{~Hz}, 2 \mathrm{H}), 3.88(\mathrm{~s}, 3 \mathrm{H}), 1.71(\mathrm{~s}, 6 \mathrm{H}) ;{ }^{13} \mathrm{C}-\mathrm{NMR}\left(\mathrm{CDCl}_{2}, 125 \mathrm{MHz}, \delta / \mathrm{ppm}\right)$ 173.6, 167.7, 163.5, 151.0, 147.2, $143.3(\mathrm{CH}), 130.1(4 \times \mathrm{CH}), 130.0,128.5,126.3(4 \times \mathrm{CH}), 125.0(2 \times \mathrm{CH}), 121.6(2 \times$ $\mathrm{CH}), 115.5(\mathrm{CH}), 114.9,84.5,52.5\left(\mathrm{CH}_{3}\right), 27.6\left(2 \times \mathrm{CH}_{3}\right)$; IR $\left(\mathrm{v} / \mathrm{cm}^{-1}\right) 1736\left(v_{\mathrm{co}}\right), 1561,1486,1283$, 1265, 1219, 1169, 1050, 760, 699; HRMS $\left(\mathrm{ESI}^{+}\right)$calcd for $\mathrm{C}_{28} \mathrm{H}_{25} \mathrm{NO}_{4} \mathrm{Na}[\mathrm{M}+\mathrm{Na}]^{+}: 462.1676$, found: 462.1656; UV-Vis $\left(\mathrm{CH}_{2} \mathrm{Cl}_{2}\right) \lambda_{\max }=483 \mathrm{~nm}\left(\varepsilon=31300 \mathrm{~L} \cdot \mathrm{mol}^{-1} \cdot \mathrm{cm}^{-1}\right)$.

2.2.14 3-(2-benzothiazolyl)-4-[2-[4-(diphenylamino)phenyl]ethenyl]-5,5-dimethyl-2-

butenolide (1i). From 1 (273 mg, $1 \mathrm{mmol}$.) and I (259 mg, $1 \mathrm{mmol}$.) in acetonitrile ( $3 \mathrm{~mL})$ and toluene $(0.5 \mathrm{~mL})$ according to general protocol 2 . The microwave irradiations were applied at $100^{\circ} \mathrm{C}$ for $3 \mathrm{~h}$. Purification by chromatography (EtOAc/Cyclohexane: 30/70) afforded the title compound 1i. Red solid $(410 \mathrm{mg}$, yield $=80 \%)$; m.p. $165-170^{\circ} \mathrm{C} ;{ }^{1} \mathrm{H}-\mathrm{NMR}\left(\mathrm{CD}_{2} \mathrm{Cl}_{2}, 500 \mathrm{MHz}, \delta / \mathrm{ppm}\right) 8.83(\mathrm{~d}, J=17.0 \mathrm{~Hz}$, $1 \mathrm{H}), 8.12(\mathrm{~d}, J=8.1 \mathrm{~Hz}, 1 \mathrm{H}), 7.99(\mathrm{~d}, J=7.8 \mathrm{~Hz}, 1 \mathrm{H}), 7.59(\mathrm{~d}, J=8.5 \mathrm{~Hz}, 2 \mathrm{H}), 7.51(\mathrm{dd}, J=7.5 \mathrm{~Hz}$, $1 \mathrm{H}), 7.43(\mathrm{dd}, J=7.5 \mathrm{~Hz}, 1 \mathrm{H}), 7.35-7.27(\mathrm{~m}, 5 \mathrm{H}), 7.20-7.10(\mathrm{~m}, 6 \mathrm{H}), 7.07(\mathrm{~d}, J=8.1 \mathrm{~Hz}, 2 \mathrm{H}), 1.83$ $(\mathrm{s}, 6 \mathrm{H}) ;{ }^{13} \mathrm{C}-\mathrm{NMR}\left(\mathrm{CD}_{2} \mathrm{Cl}_{2}, 125 \mathrm{MHz}, \delta / \mathrm{ppm}\right) 170.7,165.7,159.2,153.7,150.7,147.3,142.5(\mathrm{CH})$, 135.5, $130.1(4 \times \mathrm{CH}), 130.0(2 \times \mathrm{CH}), 129.3,126.7(\mathrm{CH}), 126.2(4 \times \mathrm{CH}), 125.9(\mathrm{CH}), 124.9(2 \times \mathrm{CH})$, $123.6(\mathrm{CH}), 122.1(\mathrm{CH}), 121.8(2 \times \mathrm{CH}), 117.5(\mathrm{CH}), 116.2,85.8,27.8\left(2 \times \mathrm{CH}_{3}\right) ; \mathrm{IR}\left(\mathrm{v} / \mathrm{cm}^{-1}\right) 1735$, 1583, 1562, 1486, 1265, 1171, 1141, 1024, 975, 761, 753, 696; HRMS (ESI ${ }^{+}$) calcd for $\mathrm{C}_{33} \mathrm{H}_{27} \mathrm{~N}_{2} \mathrm{O}_{2} \mathrm{~S}$ $[\mathrm{M}+\mathrm{H}]^{+}: 515.1788$, found: 515.1776; UV-Vis $\left(\mathrm{CH}_{2} \mathrm{Cl}_{2}\right) \lambda_{\max }=455 \mathrm{~nm}\left(\varepsilon=34900 \mathrm{~L}^{\mathrm{mol}}{ }^{-1} . \mathrm{cm}^{-1}\right)$.

2.2.15 2-((9-ethyl-9H-carbazol-3-yl)methylene)- $1 H$-indene-1,3(2H)-dione (2a). From 2 (340 $\mathrm{mg}, 1.5 \mathrm{mmol}$.) and A (175 mg, $1.2 \mathrm{mmol}$.), following general protocol 1 ( $\left.45^{\circ} \mathrm{C}, 26 \mathrm{~h}\right)$. Purification by chromatography (petroleum ether/EtOAc: $3 / 1 \mathrm{v} / \mathrm{v}$ ) afforded the title compound 2a. Orange solid (330 $\mathrm{mg}$, yield = 78\%); m.p. $230^{\circ} \mathrm{C} ;{ }^{1} \mathrm{H}-\mathrm{NMR}\left(\mathrm{CD}_{2} \mathrm{Cl}_{2}, 500 \mathrm{MHz}, \delta / \mathrm{ppm}\right) 9.46(\mathrm{~s}, 1 \mathrm{H}), 8.72(\mathrm{~d}, J=8.7 \mathrm{~Hz}$, $1 \mathrm{H}), 8.24(\mathrm{~d}, J=7.7 \mathrm{~Hz}, 1 \mathrm{H}), 8.08(\mathrm{~s}, 1 \mathrm{H}), 8.01(\mathrm{dd}, J=5.4,2.7 \mathrm{~Hz}, 1 \mathrm{H}), 7.96(\mathrm{dd}, J=5.2,2.9 \mathrm{~Hz}, 1 \mathrm{H})$, $7.88-7.75(\mathrm{~m}, 2 \mathrm{H}), 7.60-7.49(\mathrm{~m}, 3 \mathrm{H}), 7.35(\mathrm{t}, J=7.4 \mathrm{~Hz}, 1 \mathrm{H}), 4.43(\mathrm{q}, J=7.3 \mathrm{~Hz}, 2 \mathrm{H}), 1.48(\mathrm{t}, J=$ $7.3 \mathrm{~Hz}, 3 \mathrm{H}) ;{ }^{13} \mathrm{C}-\mathrm{NMR}\left(\mathrm{CD}_{2} \mathrm{Cl}_{2}, 125 \mathrm{MHz}, \delta / \mathrm{ppm}\right) 191.4(\mathrm{CO}), 190.2(\mathrm{CO}), 148.8(\mathrm{CH}), 143.8,143.0$, 141.3, 140.5, 135.4 (CH), $135.2(\mathrm{CH}), 133.7(\mathrm{CH}), 129.0(\mathrm{CH}), 127.3(\mathrm{CH}), 126.3(\mathrm{CH}), 125.4(\mathrm{CH})$, $124.1(\mathrm{CH}), 123.8(\mathrm{CH}), 123.3(\mathrm{CH}), 123.2(\mathrm{CH}), 121.4(\mathrm{CH}), 121.1(\mathrm{CH}), 110.0(\mathrm{CH}), 109.4(\mathrm{CH})$, $38.6\left(\mathrm{CH}_{2}\right), 14.2\left(\mathrm{CH}_{3}\right)$; IR $\left(\mathrm{v} / \mathrm{cm}^{-1}\right) 3059,1665\left(\mathrm{~V}_{\mathrm{co}}\right), 1571 / 1553 / 1471,1129$; HRMS $\left(\mathrm{ESI}^{+}\right)$calcd for $\mathrm{C}_{24} \mathrm{H}_{18} \mathrm{NO}_{2}[\mathrm{M}+\mathrm{H}]^{+}: 352.1332$, found: 352.1328 ; UV-Vis $\left(\mathrm{CH}_{2} \mathrm{Cl}_{2}\right) \lambda_{\max }=453 \mathrm{~nm}\left(\varepsilon=44800 \mathrm{~L}^{2} \mathrm{~mol}^{-}\right.$ $\left.{ }^{1} . \mathrm{cm}^{-1}\right)$.

2.2.16 (Z)-3-(9-ethyl-9H-carbazol-3-yl)-2-(4-nitrophenyl)acrylonitrile (2b). From 2 (450 mg, $2 \mathrm{mmol}$.) and $\mathbf{B}\left(400 \mathrm{mg}, 2.5 \mathrm{mmol}\right.$.) following general protocol $1\left(45^{\circ} \mathrm{C}, 18 \mathrm{~h}\right)$. Purification by chromatography $\left(\mathrm{CH}_{2} \mathrm{Cl}_{2}\right)$ afforded the title compound $\mathbf{2 b}$. Orange solid $(240 \mathrm{mg}$, yield $=33 \%)$; m.p. $244^{\circ} \mathrm{C} ;{ }^{1} \mathrm{H}-\mathrm{NMR}\left(\mathrm{DMSO}_{6}, 300 \mathrm{MHz}, \delta / \mathrm{ppm}\right) 8.82(\mathrm{~d}, J=1.4 \mathrm{~Hz}, 1 \mathrm{H}), 8.43(\mathrm{~s}, 1 \mathrm{H}), 8.34(\mathrm{~d}, J=8.9$ 
$\mathrm{Hz}, 2 \mathrm{H}), 8.25(\mathrm{dd}, J=8.7,1.7 \mathrm{~Hz}, 1 \mathrm{H}), 8.14(\mathrm{~d}, J=7.7 \mathrm{~Hz}, 1 \mathrm{H}), 8.04(\mathrm{~d}, J=8.9 \mathrm{~Hz}, 2 \mathrm{H}), 7.82(\mathrm{~d}, J=$ $8.7 \mathrm{~Hz}, 1 \mathrm{H}), 7.70(\mathrm{~d}, J=8.2 \mathrm{~Hz}, 1 \mathrm{H}), 7.54(\mathrm{t}, J=7.7 \mathrm{~Hz}, 1 \mathrm{H}), 7.31(\mathrm{t}, J=7.5 \mathrm{~Hz}, 1 \mathrm{H}), 4.51(\mathrm{q}, J=7.1$ $\mathrm{Hz}, 2 \mathrm{H}), 1.35(\mathrm{t}, J=7.1 \mathrm{~Hz}, 3 \mathrm{H}) ;{ }^{13} \mathrm{C}-\mathrm{NMR}\left(\mathrm{DMSO}-\mathrm{d}_{6}, 101 \mathrm{MHz}, \delta / \mathrm{ppm}\right) 147.6(\mathrm{CH}), 146.8,141.4$, 141.1, 140.3, $127.2(\mathrm{CH}), 126.8(\mathrm{CH}), 126.4(\mathrm{CH}), 124.5(2 \times \mathrm{CH}), 124.2,123.6(2$ x CH$), 122.5,122.1$, $120.4(\mathrm{CH}), 120.1(\mathrm{CH}), 118.5,110.0(\mathrm{CH}), 103.8(\mathrm{CN}), 37.4\left(\mathrm{CH}_{2}\right), 13.8\left(\mathrm{CH}_{3}\right)$; IR $\left(\mathrm{v} / \mathrm{cm}^{-1}\right) 2208$ $\left(v_{\mathrm{CN}}\right), 1576 / 1515 / 1472,1334,1231,1159$; HRMS $\left(\mathrm{ESI}^{+}\right)$calcd for $\mathrm{C}_{23} \mathrm{H}_{17} \mathrm{~N}_{3} \mathrm{O}_{2} \mathrm{Na}[\mathrm{M}+\mathrm{Na}]^{+}: 390.1213$, found: 390.1207 ; UV-Vis $\left(\mathrm{CH}_{2} \mathrm{Cl}_{2}\right) \lambda_{\max }=405 \mathrm{~nm}\left(\varepsilon=21300 \mathrm{~L} \cdot \mathrm{mol}^{-1} . \mathrm{cm}^{-1}\right)$.

2.2.17 (Z)-3-(9-ethyl-9H-carbazol-3-yl)-2-(pyridine-4-yl)acrylonitrile (2c). From 2 (300 mg, $1.35 \mathrm{mmol}$.) and $\mathbf{C}\left(250 \mathrm{mg}, 1.5 \mathrm{mmol}\right.$.) following general protocol $2\left(60^{\circ} \mathrm{C}, 48 \mathrm{~h}\right)$. Purification by chromatography (petroleum ether, EtOAc: 6/1 v/v) afforded the title compound. Orange solid (130 mg, yield $=30 \%)$; m.p. $216^{\circ} \mathrm{C} ;{ }^{1} \mathrm{H}-\mathrm{NMR}\left(\mathrm{DMSO}_{6}, 300 \mathrm{MHz}, \delta / \mathrm{ppm}\right) 8.84-8.64(\mathrm{~m}, 3 \mathrm{H}), 8.45(\mathrm{~s}, 1 \mathrm{H})$, $8.24(\mathrm{dd}, J=8.8,1.6 \mathrm{~Hz}, 1 \mathrm{H}), 8.14(\mathrm{~d}, J=7.7 \mathrm{~Hz}, 1 \mathrm{H}), 7.85-7.66(\mathrm{~m}, 4 \mathrm{H}), 7.59-7.49$ (m, 1H), 7.30 $(\mathrm{t}, J=7.4 \mathrm{~Hz}, 1 \mathrm{H}), 4.49(\mathrm{q}, J=7.1 \mathrm{~Hz}, 2 \mathrm{H}), 1.33(\mathrm{t}, J=7.1 \mathrm{~Hz}, 3 \mathrm{H}),{ }^{13} \mathrm{C}-\mathrm{NMR}\left(\mathrm{DMSO}-\mathrm{d}_{6}, 75 \mathrm{MHz}\right.$, $\delta / \mathrm{ppm}) 150.4,147.0,141.8,141.3,140.2,127.1,126.7,124.0,123.5,122.4,122.0,120.3,120.001$, 119.4, 118.0, 109.9, 109.8, 103.3, 37.3, 13.7; IR $\left(\mathrm{v} \mathrm{cm}^{-1}\right)$ 2926, $2214\left(\mathrm{v}_{\mathrm{CN}}\right), 1628,1573 / 1492,1234$; HRMS (ESI ${ }^{+}$) calcd for $\mathrm{C}_{22} \mathrm{H}_{18} \mathrm{~N}_{3}[\mathrm{M}+\mathrm{H}]^{+}: 324.1495$, found: 324.1483 ; UV-Vis $\left(\mathrm{CH}_{2} \mathrm{Cl}_{2}\right) \lambda_{\max }=389 \mathrm{~nm}$ $\left(\varepsilon=25000 \mathrm{~L} \cdot \mathrm{mol}^{-1} \cdot \mathrm{cm}^{-1}\right)$.

2.2.18 (E)-2-(benzo[d]thiazol-2-yl)-3-(9-ethyl-9H-carbazol-3-yl)acrylonitrile (2d). From 2 (400 mg, $1.8 \mathrm{mmol}$.) and $\mathbf{D}\left(375 \mathrm{mg}, 2.5 \mathrm{mmol}\right.$.) following general protocol $2\left(40{ }^{\circ} \mathrm{C}, 18 \mathrm{~h}\right)$. Purification by chromatography $\left(\mathrm{CH}_{2} \mathrm{Cl}_{2}\right)$ afforded the title compound $2 \mathrm{~d}$. Orange solid $(680 \mathrm{mg}$, yield $=57 \%)$. m.p. $230^{\circ} \mathrm{C} ;{ }^{1} \mathrm{H}-\mathrm{NMR}\left(\mathrm{CD}_{2} \mathrm{Cl}_{2}, 500 \mathrm{MHz}, \delta / \mathrm{ppm}\right) 8.80(\mathrm{~s}, 1 \mathrm{H}), 8.37(\mathrm{~s}, 1 \mathrm{H}), 8.28(\mathrm{dd}, J=8.7,1.5 \mathrm{~Hz}, 1 \mathrm{H})$, $8.19(\mathrm{~d}, J=7.8 \mathrm{~Hz}, 1 \mathrm{H}), 8.06(\mathrm{~d}, J=8.1 \mathrm{~Hz}, 1 \mathrm{H}), 7.95(\mathrm{~d}, J=8.0 \mathrm{~Hz}, 1 \mathrm{H}), 7.62-7.48(\mathrm{~m}, 4 \mathrm{H}), 7.44(\mathrm{t}$, $J=7.6 \mathrm{~Hz}, 1 \mathrm{H}), 7.34(\mathrm{t}, J=7.0 \mathrm{~Hz}, 1 \mathrm{H}), 4.44(\mathrm{q}, J=7.3 \mathrm{~Hz}, 2 \mathrm{H}), 1.48(\mathrm{t}, J=7.3 \mathrm{~Hz}, 3 \mathrm{H}) ;{ }^{13} \mathrm{C} \mathrm{NMR}$ (125 MHz, $\left.\mathrm{CH}_{2} \mathrm{Cl}_{2}, \delta / \mathrm{ppm}\right) 164.8,154.3,148.8(\mathrm{CH}), 142.7,141.2,135.2,128.7(\mathrm{CH}), 127.2(2 \times \mathrm{CH})$, $126.1(\mathrm{CH}), 124.7(\mathrm{CH}), 124.0,123.6(\mathrm{CH}), 123.3,122.1(\mathrm{CH}), 121.3(\mathrm{CH}), 120.8(\mathrm{CH}), 118.0,109.8$ $(2 \times \mathrm{CH}), 101.8,38.5\left(\mathrm{CH}_{2}\right), 14.2\left(\mathrm{CH}_{3}\right)$; IR $\left({\left.\mathrm{v} / \mathrm{cm}^{-1}\right)}^{-1} 2361,1576 / 1559 / 1469,1233,1128 ; \mathrm{HRMS}\left(\mathrm{ESI}^{+}\right)\right.$ calcd for $\mathrm{C}_{24} \mathrm{H}_{18} \mathrm{~N}_{3} \mathrm{~S}[\mathrm{M}+\mathrm{H}]^{+}$: 380.1216, found: 380.1207; UV-Vis $\left(\mathrm{CH}_{2} \mathrm{Cl}_{2}\right) \lambda_{\max }=417 \mathrm{~nm}(\varepsilon=$ $\left.20300 \mathrm{~L} \cdot \mathrm{mol}^{-1} \cdot \mathrm{cm}^{-1}\right)$.

2.2.19 (E)-2-(3-cyano-4-(2-(9-ethyl-9H-carbazol-3-yl)vinyl)-5,5'-dimethylfuran-2(5H)ylidene)malononitrile (2f). From $2(340 \mathrm{mg}, 1.5 \mathrm{mmol}$.) and $\mathbf{F}(380 \mathrm{mg}, 1.9 \mathrm{mmol}$.) following general protocol $1\left(45^{\circ} \mathrm{C}, 48 \mathrm{~h}\right)$. Purification by chromatography $\left(\mathrm{CH}_{2} \mathrm{Cl}_{2} /\right.$ pentane: $1 / 1$ then $\left.2 / 1 \mathrm{v} / \mathrm{v}\right)$ afforded the title compound 2f. Red solid $(180 \mathrm{mg}$, yield $=30 \%)$; m.p. $230{ }^{\circ} \mathrm{C} ;{ }^{1} \mathrm{H}-\mathrm{NMR}$ (DMSO- $d_{6}, 400 \mathrm{MHz}$, $\delta / \mathrm{ppm}) 8.79(\mathrm{~d}, J=1.3 \mathrm{~Hz}, 1 \mathrm{H}), 8.27(\mathrm{~d}, J=7.7 \mathrm{~Hz}, 1 \mathrm{H}), 8.18(\mathrm{~d}, J=16.2 \mathrm{~Hz}, 1 \mathrm{H}), 8.07(\mathrm{dd}, J=8.8$, $1.5 \mathrm{~Hz}, 1 \mathrm{H}), 7.76(\mathrm{~d}, J=8.7 \mathrm{~Hz}, 1 \mathrm{H}), 7.70(\mathrm{~d}, J=8.2 \mathrm{~Hz}, 1 \mathrm{H}), 7.60-7.48(\mathrm{~m}, 1 \mathrm{H}), 7.31(\mathrm{dd}, J=11.1$, $3.8 \mathrm{~Hz}, 1 \mathrm{H}), 7.26(\mathrm{~d}, J=16.2 \mathrm{~Hz}, 1 \mathrm{H}), 4.50(\mathrm{q}, J=7.1 \mathrm{~Hz}, 2 \mathrm{H}), 1.83(\mathrm{~s}, 6 \mathrm{H}), 1.34(\mathrm{t}, J=7.1 \mathrm{~Hz}, 3 \mathrm{H})$; ${ }^{13} \mathrm{C}$ NMR (DMSO- $\left.d_{6}, 101 \mathrm{MHz}, \delta / \mathrm{ppm}\right) 177.4,175.8,149.9(\mathrm{CH}), 142.4,140.3,127.9(\mathrm{CH}), 126.9$ $(\mathrm{CH}), 125.6,123.9(\mathrm{CH}), 123.1,122.3,121.0(\mathrm{CH}), 120.3(\mathrm{CH}), 113.1,112.3,111.9(\mathrm{CH}), 111.5,110.2$ $(\mathrm{CH}), 110.1(\mathrm{CH}), 99.0,96.0,53.0,37.4\left(\mathrm{CH}_{2}\right), 25.4\left(2 \mathrm{x} \mathrm{CH}_{3}\right), 13.9\left(\mathrm{CH}_{3}\right) ; \mathrm{IR}\left(\mathrm{v} / \mathrm{cm}^{-1}\right) 2219(\mathrm{VCN})$, 1542/1508/1490, 1394, 1106; HRMS (ESI ${ }^{+}$) calcd for $\mathrm{C}_{26} \mathrm{H}_{20} \mathrm{~N}_{4} \mathrm{ONa}[\mathrm{M}+\mathrm{Na}]^{+}$: 427.1529, found: 427.1515 (calcd.); UV-Vis $\left(\mathrm{CH}_{2} \mathrm{Cl}_{2}\right) \lambda_{\max }=510 \mathrm{~nm}\left(\varepsilon=24000 \mathrm{~L} \cdot \mathrm{mol}^{-1} \cdot \mathrm{cm}^{-1}\right)$.

2.2.20 (E)-methyl-4-(2-(9-ethyl-9H-carbazol-3-yl)vinyl)-5,5'-dimethyl-2-oxo-2,5-

dihydrofuran-3-carboxylate (2h). From 2 (470 mg, $2.1 \mathrm{mmol}$.) and $\mathbf{H}(330 \mathrm{mg}, 1.8 \mathrm{mmol}$.) following general protocol $1\left(50^{\circ} \mathrm{C}, 48 \mathrm{~h}\right)$. Purification by chromatography (Petroleum ether, EtOAc: 6/1 v/v) afforded the title compound $2 \mathrm{~h}$. Orange solid $(450 \mathrm{mg}$, yield $=65 \%)$; m.p. $218^{\circ} \mathrm{C} ;{ }^{1} \mathrm{H}-\mathrm{NMR}\left(\mathrm{CD}_{2} \mathrm{Cl}_{2}\right.$, $400 \mathrm{MHz}, \delta / \mathrm{ppm}) 8.35(\mathrm{~s}, 1 \mathrm{H}), 8.16(\mathrm{~d}, J=7.7 \mathrm{~Hz}, 1 \mathrm{H}), 7.97(\mathrm{~d}, J=16.9 \mathrm{~Hz}, 1 \mathrm{H}), 7.81(\mathrm{~d}, J=8.6 \mathrm{~Hz}$, $1 \mathrm{H}), 7.59-7.40(\mathrm{~m}, 4 \mathrm{H}), 7.30(\mathrm{t}, J=7.3 \mathrm{~Hz}, 1 \mathrm{H}), 4.40(\mathrm{q}, J=7.1 \mathrm{~Hz}, 2 \mathrm{H}), 3.94(\mathrm{~s}, 3 \mathrm{H}), 1.79(\mathrm{~s}, 6 \mathrm{H})$, $1.45(\mathrm{t}, J=7.2 \mathrm{~Hz}, 3 \mathrm{H}) ;{ }^{13} \mathrm{C}-\mathrm{NMR}\left(\mathrm{CD}_{2} \mathrm{Cl}_{2}, 101 \mathrm{MHz}, \delta / \mathrm{ppm}\right) 174.1,167.95,163.8,145.4(\mathrm{CH}), 142.3$, 141.2, $127.2(\mathrm{CH}), 126.4(\mathrm{CH}), 124.1,123.4,122.3(\mathrm{CH}), 121.2(\mathrm{CH}), 120.5(\mathrm{CH}), 115.3(\mathrm{CH}), 114.3$, $109.8(\mathrm{CH}), 84.7,52.7(\mathrm{CH}), 38.6,27.8\left(2 \times \mathrm{CH}_{3}\right), 14.3\left(\mathrm{CH}_{3}\right) ; \mathrm{IR}\left(\mathrm{v} / \mathrm{cm}^{-1}\right)$ 2364, 1759, 1572/1542/1490, 1333, 1213, 1123; HRMS $\left(\mathrm{ESI}^{+}\right)$calcd for $\mathrm{C}_{24} \mathrm{H}_{23} \mathrm{NO}_{4} \mathrm{Na}[\mathrm{M}+\mathrm{Na}]^{+}: 412.1519$, found: 
412.1511; UV-vis $\left(\mathrm{CH}_{2} \mathrm{Cl}_{2}\right) \lambda_{\max }=413 \mathrm{~nm}\left(\varepsilon=22590 \mathrm{~L} \cdot \mathrm{mol}^{-1} . \mathrm{cm}^{-1}\right)$.

2.2.21 (E)-3-(benzo[d]thiazol-2-yl)-4-(2-(9-ethyl-9H-carbazol-3-yl)vinyl)-5,5'-

dimethylfuran-2(5H)-one (2i). From $2(300 \mathrm{mg}, 1.3 \mathrm{mmol}$.) and I (420 mg, $1.6 \mathrm{mmol}$.) following general protocol $1\left(70^{\circ} \mathrm{C}, 48 \mathrm{~h}\right)$. Purification by chromatography (Petroleum ether, EtOAc: 6/1 v/v) afforded the title compound 2i. Orange solid $(240 \mathrm{mg}$, yield $=78 \%)$; m.p. $223^{\circ} \mathrm{C} ;{ }^{1} \mathrm{H} \mathrm{NMR}(500 \mathrm{MHz}$, $\left.\mathrm{CD}_{2} \mathrm{Cl}_{2}, \delta / \mathrm{ppm}\right) .89(\mathrm{~d}, J=16.9 \mathrm{~Hz}, 1 \mathrm{H}), 8.35(\mathrm{~d}, J=1.1 \mathrm{~Hz}, 1 \mathrm{H}), 8.14(\mathrm{~d}, J=8.1 \mathrm{~Hz}, 1 \mathrm{H}), 8.08(\mathrm{~d}, J=$ $7.7 \mathrm{~Hz}, 1 \mathrm{H}), 8.02-7.85(\mathrm{~m}, 1 \mathrm{H}), 7.81(\mathrm{dd}, J=8.6,1.5 \mathrm{~Hz}, 1 \mathrm{H}), 7.58-7.30(\mathrm{~m}, 6 \mathrm{H}), 7.21(\mathrm{dd}, J=10.9$, $3.8 \mathrm{~Hz}, 1 \mathrm{H}), 4.31(\mathrm{q}, J=7.3 \mathrm{~Hz}, 2 \mathrm{H}), 1.78(\mathrm{~s}, 6 \mathrm{H}), 1.36(\mathrm{t}, J=7.3 \mathrm{~Hz}, 3 \mathrm{H}) ;{ }^{13} \mathrm{C}$ NMR $(125 \mathrm{MHz}$, $\left.\mathrm{CD}_{2} \mathrm{Cl}_{2}, \delta / \mathrm{ppm}\right) 170.7,165.9,159.2,153.6,144.3,141.9,141.0,135.5,127.4,126.9,126.6,126.3$, $125.8,125.8,123.9,123.5,123.2,122.1,122.0,121.8,120.9,120.2,117.0,115.9,109.8,109.6,87.3$, 85.7, 38.3, 27.7, 24.7, 14.0, 13.7; IR (v/cm $\left.{ }^{-1}\right)$ 2364, 1748, 1559/1471/1457, 1233, 1122; HRMS (ESI $\left.{ }^{+}\right)$ calcd for $\mathrm{C}_{29} \mathrm{H}_{24} \mathrm{~N}_{2} \mathrm{O}_{2} \mathrm{SNa}[\mathrm{M}+\mathrm{Na}]^{+}$: 487.1451, found 487.1436; UV-Vis $\left(\mathrm{CH}_{2} \mathrm{Cl}_{2}\right) \lambda_{\max }=452 \mathrm{~nm}$ $\left(\varepsilon=22600 \mathrm{~L} \cdot \mathrm{mol}^{-1} \cdot \mathrm{cm}^{-1}\right)$.

2.2.22 $(E)$-4-(2-(9-ethyl-9H-carbazol-3-yl)vinyl)-5,5'-dimethyl-3-(phenylsulfonyl)furan$\mathbf{2}(\mathbf{5 H})$-one (2j). From 2 (320 mg, $1.4 \mathrm{mmol}$.) and $\mathbf{J}$ (320 mg, $1.2 \mathrm{mmol}$.) following general protocol 1 $\left(50^{\circ} \mathrm{C}, 48 \mathrm{~h}\right)$. Purification by chromatography (petroleum ether, EtOAc: 6/1 v/v) afforded the title compound 2j. Orange solid (165 mg, yield $=29 \%)$; m.p. $224^{\circ} \mathrm{C} ;{ }^{1} \mathrm{H}-\mathrm{NMR}\left(\mathrm{CD}_{2} \mathrm{Cl}_{2}, 500 \mathrm{MHz}, \delta / \mathrm{ppm}\right)$ $8.4(\mathrm{~s}, 1 \mathrm{H}), 8.35(\mathrm{~d}, J=16.8 \mathrm{~Hz}, 1 \mathrm{H}), 8.18(\mathrm{~d}, J=7.6 \mathrm{~Hz}, 1 \mathrm{H}), 8.10(\mathrm{~d}, J=7.6 \mathrm{~Hz}, 2 \mathrm{H}), 7.91(\mathrm{~d}, J=8.5 \mathrm{~Hz}$, 1H), 7.67 (t, J=7.3 Hz, 1H), 7.60-7.52 (m, 6H), 7.32 (t, J=7.3 Hz, 1H), 4.43 (q, J=7.2 Hz, 2H), 1.77 (s, $6 \mathrm{H}), 1.48(\mathrm{t}, J=7.2 \mathrm{~Hz}, 3 \mathrm{H}) ;{ }^{13} \mathrm{C} \mathrm{NMR}\left(125 \mathrm{MHz}, \mathrm{CD}_{2} \mathrm{Cl}_{2}, \delta / \mathrm{ppm}\right) 172.2,165.6,147.3,142.6,141.2$, 141.0, 134.5, 129.6, 128.9, 127.2, 126.6, 124.2, 123.3, 123.0, 121.7, 121.2, 120.6, 113.2, 110.1, 109.8, 85.7, 38.6, 27.8, 14.2; IR $\left(\mathrm{v} / \mathrm{cm}^{-1}\right)$ 1748, 1583/1529/1472, 1330, 1234, 1148; HRMS (ESI $\left.{ }^{+}\right)$calcd for $\mathrm{C}_{28} \mathrm{H}_{25} \mathrm{NO}_{4} \mathrm{SNa} \quad[\mathrm{M}+\mathrm{Na}]^{+}: \quad 494.1397$, found 494.1378; UV-Vis $\left(\mathrm{CH}_{2} \mathrm{Cl}_{2}\right) \quad \lambda_{\max }=439 \mathrm{~nm}$ $\left(\varepsilon=26100 \mathrm{~L} \cdot \mathrm{mol}^{-1} \cdot \mathrm{cm}^{-1}\right)$.

2.2.23 (Z)-3-(4-(9H-carbazol-9-yl)phenyl)-2-(4-nitrophenyl)acrylonitrile (3b). From 3 (500 $\mathrm{mg}, 1.85 \mathrm{mmol}$.) and $\mathbf{B}\left(450 \mathrm{mg}, 2.78 \mathrm{mmol}\right.$.) following general protocol $1\left(40^{\circ} \mathrm{C}, 18 \mathrm{~h}\right)$. Purification by chromatography (Petroleum ether/EtOAc: 6/1 v/v) afforded the title compound 3b. Orange solid (430 mg, yield $=56 \%)$; m.p. $223^{\circ} \mathrm{C} ;{ }^{1} \mathrm{H}-\mathrm{NMR}\left(\mathrm{CD}_{2} \mathrm{Cl}_{2}, 500 \mathrm{MHz}, \delta / \mathrm{ppm}\right) 8.34(\mathrm{~d}, J=8.0 \mathrm{~Hz}, 2 \mathrm{H}), 8.23(\mathrm{~d}, J$ $=7.8 \mathrm{~Hz}, 2 \mathrm{H}), 8.17(\mathrm{~d}, J=7.7 \mathrm{~Hz}, 2 \mathrm{H}), 7.93(\mathrm{~d}, J=7.9 \mathrm{~Hz}, 2 \mathrm{H}), 7.85-7.76(\mathrm{~m}, 3 \mathrm{H}), 7.55(\mathrm{~d}, J=8.2$ $\mathrm{Hz}, 2 \mathrm{H}), 7.46(\mathrm{t}, J=7.6 \mathrm{~Hz}, 2 \mathrm{H}), 7.33(\mathrm{t}, J=7.4 \mathrm{~Hz}, 2 \mathrm{H}) ;{ }^{13} \mathrm{C}-\mathrm{NMR}\left(\mathrm{CD}_{2} \mathrm{Cl}_{2}, 125 \mathrm{MHz}, \delta / \mathrm{ppm}\right) 144.8$, 140.8, 131.9, 127.4, 126.8, 124.9, 124.3, 121.2, 121.0, 117.7, 110.4; IR (v/cm $\left.{ }^{-1}\right) 2219,1588 / 1511 / 1448$, 1220, 1170; HRMS (EI) calcd for $\mathrm{C}_{27} \mathrm{H}_{17} \mathrm{~N}_{3} \mathrm{O}_{2}[\mathrm{M}]^{+} \cdot: 416.1315$, found: 415.1317 ; UV-Vis $\left(\mathrm{CH}_{2} \mathrm{Cl}_{2}\right) \lambda_{\max }$ $=404 \mathrm{~nm}\left(\varepsilon=21110 \mathrm{~L} \cdot \mathrm{mol}^{-1} \cdot \mathrm{cm}^{-1}\right)$.

2.2.24 (E)-2-(4-(4-(9H-carbazol-9-yl)styryl)-3-cyano-5,5'-dimethylfuran-2(5H)-

ylidene)malononitrile (3f). From 3 (270 mg, $1 \mathrm{mmol}$.) and F (240 mg, 1,2 mmol.) following general protocol $1\left(50^{\circ} \mathrm{C}, 18 \mathrm{~h}\right)$. Purification by chromatography (Pentane/EtOAc: $\left.70 / 30 \mathrm{v} / \mathrm{v}\right)$ afforded the title compound 3f. Black solid $\left(175 \mathrm{mg}\right.$, yield $=39 \%$ ); m.p. $>260{ }^{\circ} \mathrm{C}$; ${ }^{1} \mathrm{H}-\mathrm{NMR}$ (DMSO-d6, $500 \mathrm{MHz}$, $\delta / \mathrm{ppm}) 8.28(\mathrm{~d}, J=7.8 \mathrm{~Hz}, 2 \mathrm{H}), 8.22(\mathrm{~d}, J=8.5 \mathrm{~Hz}, 2 \mathrm{H}), 8.07(\mathrm{~d}, J=16.5 \mathrm{~Hz}, 1 \mathrm{H}), 7.83(\mathrm{~d}, J=8.6 \mathrm{~Hz}$, 2H), 7.49 (ddd, $J=13.3,9.4,4.7 \mathrm{~Hz}, 4 \mathrm{H}), 7.39-7.27(\mathrm{~m}, 3 \mathrm{H}), 1.86(\mathrm{~s}, 6 \mathrm{H}),{ }^{13} \mathrm{C}-\mathrm{NMR}$ (DMSO- 6,125

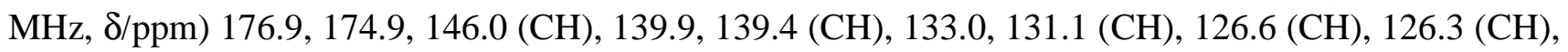
123.0 (CH), 120.5, 115.7, 112.5, 111.7, 110.7, $109.7(\mathrm{CH}), 99.6(\mathrm{CN}), 99.3(2 \times \mathrm{CN})$, 54.4, $25.0\left(\mathrm{CH}_{3}\right)$; IR $\left(\mathrm{V} / \mathrm{cm}^{-1}\right)$ 2989, 2223, 2202, 1567, 1553, 1165, 745; HRMS (ESI ${ }^{+}$calcd for $\mathrm{C}_{30} \mathrm{H}_{20} \mathrm{~N}_{4} \mathrm{ONa}[\mathrm{M}+\mathrm{Na}]^{+}$: 475.1529, found: 475.1516 ; UV-Vis $\left(\mathrm{CH}_{2} \mathrm{Cl}_{2}\right) \lambda_{\max }=481 \mathrm{~nm}\left(\varepsilon=29700 \mathrm{~L} \cdot \mathrm{mol}^{-1} \cdot \mathrm{cm}^{-1}\right)$.

2.2.25 (E)-4-(4-(9H-carbazol-9-yl)styryl)-5,5'-dimethyl-2-oxo-2,5 dihydrofuran-3carbonitrile (3g). From 3 (270 mg, 1 mmol.) and G (150 mg, $1 \mathrm{mmol}$.) following general protocol 1 $\left(70^{\circ} \mathrm{C}, 4 \mathrm{~h}\right.$. Purification by chromatography (EtOAc/Pentane: 30/70) afforded the title compound 3g. Yellow solid $(290 \mathrm{mg}$, yield $=72 \%)$; m.p. $203^{\circ} \mathrm{C} ;{ }^{1} \mathrm{H}-\mathrm{NMR}\left(\mathrm{CDCl}_{3}, 500 \mathrm{MHz}, \delta / \mathrm{ppm}\right) 8.16(\mathrm{~d}, J=7.7$ $\mathrm{Hz}, 2 \mathrm{H}), 7.88(\mathrm{~d}, J=8.4 \mathrm{~Hz}, 2 \mathrm{H}), 7.83(\mathrm{~d}, J=16.5 \mathrm{~Hz}, 1 \mathrm{H}), 7.73(\mathrm{~d}, J=8.4 \mathrm{~Hz}, 2 \mathrm{H}), 7.54-7.41(\mathrm{~m}$, $4 \mathrm{H}), 7.33(\mathrm{t}, J=7.4 \mathrm{~Hz}, 2 \mathrm{H}), 6.98(\mathrm{~d}, J=16.4 \mathrm{~Hz}, 1 \mathrm{H}), 1.74(\mathrm{~s}, 6 \mathrm{H}) ;{ }^{13} \mathrm{C}-\mathrm{NMR}\left(\mathrm{CDCl}_{3}, 125 \mathrm{MHz}\right.$,

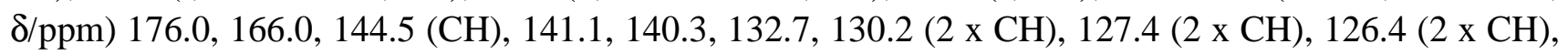


124.0, $120.9(2 \times \mathrm{CH}), 120.7(2 \times \mathrm{CH}), 115.4(\mathrm{CH}), 112.2,109.9(2 \times \mathrm{CH}), 99.4,87.0,26.1\left(2 \times \mathrm{CH}_{3}\right)$; IR $\left(\mathrm{V} / \mathrm{cm}^{-1}\right) 2233\left(\mathrm{~V}_{\mathrm{CN}}\right), 1763,1585,1516,1449,1334,1276,1231,1219,1172,1069,750,723$; HRMS $\left(\mathrm{ESI}^{+}\right)$calcd for $\mathrm{C}_{27} \mathrm{H}_{20} \mathrm{~N}_{2} \mathrm{O}_{2} \mathrm{Na}[\mathrm{M}+\mathrm{Na}]^{+}: 427.1417$, found: 427.1413; UV-Vis $\left(\mathrm{CH}_{2} \mathrm{Cl}_{2}\right) \lambda_{\max }=421 \mathrm{~nm}$ $\left(\varepsilon=21070 \mathrm{~L} \cdot \mathrm{mol}^{-1} \cdot \mathrm{cm}^{-1}\right)$.

2.2.26 (E)-methyl-4-(4-(9H-carbazol-9-yl)styryl)-5,5'-dimethyl-2-oxo-2,5-dihydrofuran-3carboxylate (3h). From 3 (270 mg, 1 mmol.) and $\mathbf{H}(200 \mathrm{mg}, 1 \mathrm{mmol}$.) following general protocol 1 $\left(80^{\circ} \mathrm{C}, 12 \mathrm{~h}\right)$. Purification by chromatography (Cyclohexane/EtOAc: 80/20 v/v) afforded the title compound 3h. Yellow solid (330 mg, yield $=78 \%$ ); m.p. $174{ }^{\circ} \mathrm{C} ;{ }^{1} \mathrm{H}-\mathrm{NMR}\left(\mathrm{CD}_{2} \mathrm{Cl}_{2}, 500 \mathrm{MHz}, \delta / \mathrm{ppm}\right)$ $8.16(\mathrm{~d}, J=7.7 \mathrm{~Hz}, 2 \mathrm{H}), 7.96(\mathrm{~d}, J=17.0 \mathrm{~Hz}, 1 \mathrm{H}), 7.88(\mathrm{~d}, J=8.3 \mathrm{~Hz}, 2 \mathrm{H}), 7.70(\mathrm{~d}, J=8.3 \mathrm{~Hz}, 2 \mathrm{H})$, $7.50(\mathrm{~d}, J=8.2 \mathrm{~Hz}, 2 \mathrm{H}), 7.45(\mathrm{t}, J=7.5 \mathrm{~Hz}, 2 \mathrm{H}), 7.40-7.29(\mathrm{~m}, 3 \mathrm{H}), 3.94(\mathrm{~s}, 3 \mathrm{H}), 1.78(\mathrm{~s}, 6 \mathrm{H}) ;{ }^{13} \mathrm{C}-$ NMR $\left(\mathrm{CD}_{2} \mathrm{Cl}_{2}, 125 \mathrm{MHz}, \delta / \mathrm{ppm}\right)$ 158.2, 152.9, 148.7, $127.6(\mathrm{CH}), 126.5,125.8,120.1,115.6(2 \times \mathrm{CH})$, $113.2(2 \times \mathrm{CH}), 112.2(2 \times \mathrm{CH}), 106.5(2 \times \mathrm{CH}), 106.4(2 \times \mathrm{CH}), 104.3(\mathrm{CH}), 102.9,95.9(2 \times \mathrm{CH})$, 86.0, 70.3, $38.3\left(\mathrm{CH}_{3}\right), 12.9\left(2 \times \mathrm{CH}_{3}\right)$; IR $\left(\mathrm{v} / \mathrm{cm}^{-1}\right) 1748$ (Vco), 1587, 1515, 1450, 1230, 1215, 1049, 746, 723; HRMS (ESI ${ }^{+}$calcd for $\mathrm{C}_{28} \mathrm{H}_{23} \mathrm{NO}_{4} \mathrm{Na}[\mathrm{M}+\mathrm{Na}]^{+}: 460.1519$, found: 460.1506; UV-Vis $\left(\mathrm{CH}_{2} \mathrm{Cl}_{2}\right)$ $\lambda_{\max }=397 \mathrm{~nm}\left(\varepsilon=23200 \mathrm{~L} \cdot \mathrm{mol}^{-1} \cdot \mathrm{cm}^{-1}\right)$.

2.2.27 (E)-4-(4-(9H-carbazol-9-yl)styryl)-3-(benzo[d]thiazol-2-yl)-5, 5'- dimethylfuran2(5H)-one (3i). From 3 (270 mg, $1 \mathrm{mmol}$.) and I (260 mg, $1 \mathrm{mmol}$.) following general protocol 1 $\left(80^{\circ} \mathrm{C}, 24 \mathrm{~h}\right)$. Purification by chromatography (Pentane/EtOAc: $90 / 10 \mathrm{v} / \mathrm{v}$ ) afforded the title compound 3i. Orange solid $(445 \mathrm{mg}$, yield $=86 \%)$; m.p. $142{ }^{\circ} \mathrm{C} ;{ }^{1} \mathrm{H}-\mathrm{NMR}\left(\mathrm{CDCl}_{3}, 500 \mathrm{MHz}, \delta / \mathrm{ppm}\right) 8.20(\mathrm{dd}, J=$ $8.0 \mathrm{~Hz}, 1 \mathrm{H}), 8.17(\mathrm{~d}, J=8.2 \mathrm{~Hz}, 2 \mathrm{H}), 8.01(\mathrm{~d}, J=7.9 \mathrm{~Hz}, 1 \mathrm{H}), 7.94(\mathrm{~d}, J=8.3 \mathrm{~Hz}, 2 \mathrm{H}), 7.72(\mathrm{~d}, J=8.4$ $\mathrm{Hz}, 2 \mathrm{H}), 7.62-7.38(\mathrm{~m}, 7 \mathrm{H}), 7.33(\mathrm{t}, J=7.4 \mathrm{~Hz}, 2 \mathrm{H}), 1.90(\mathrm{~s}, 6 \mathrm{H}) ;{ }^{13} \mathrm{C}-\mathrm{NMR}\left(\mathrm{CDCl}_{3}, 125 \mathrm{MHz}\right.$, $\delta / \mathrm{ppm}) 170.0,164.0,158.0,153.0,140.5(\mathrm{CH}), 140.3,139.4,135.2,134.6,129.5(2 \mathrm{x} \mathrm{CH}), 127.2(2 \mathrm{x}$ $\mathrm{CH}), 126.3(\mathrm{CH}), 126.1(2 \times \mathrm{CH}), 125.6(\mathrm{CH}), 123.7,123.6(\mathrm{CH}), 123.2(\mathrm{CH}), 120.2(2 \times \mathrm{CH}), 117.9$, $109.7(2 \times \mathrm{CH}), 99.9(\mathrm{CH}), 85.4,27.3\left(2 \times \mathrm{CH}_{3}\right)$; IR $\left(\mathrm{v} / \mathrm{cm}^{-1}\right) 1736,1595,1574,1513,1448,1333,1313$, 1269, 1226, 1170, 1143, 1026, 748, 723; HRMS (ESI ${ }^{+}$) calcd for $\mathrm{C}_{33} \mathrm{H}_{25} \mathrm{~N}_{2} \mathrm{O}_{2} \mathrm{~S}[\mathrm{M}+\mathrm{H}]^{+}:$513.1631, found: 513.1631 ; UV-Vis $\left(\mathrm{CH}_{2} \mathrm{Cl}_{2}\right) \lambda_{\max }=417 \mathrm{~nm}\left(\varepsilon=20630 \mathrm{~L} \cdot \mathrm{mol}^{-1} \cdot \mathrm{cm}^{-1}\right)$.

\subsection{Crystallography}

Single crystals suitable for X-ray diffraction were grown by slow diffusion of diisopropylether in concentrated chloroform solution. CCDC 1560148 (1a), 1560149 (1)), 1564219 (1d), 837775 (1e), $1578107(\mathbf{1 g}), 1560145(\mathbf{1 i}), 1560146(\mathbf{2 b}), 1564223(\mathbf{2 i}), 1560147(\mathbf{2 j})$ and $1560150(\mathbf{3 f})$ contains the supplementary crystallographic data for this paper. These data can be obtained free of charge from The Cambridge Crystallographic Data Centre via www.ccdc.cam.ac.uk/data_request/cif.

\subsection{Spectroscopic measurements and solid-state fluorescence}

Absorption spectra were recorded on a JASCO V670 spectrophotometer. Fluorescence spectra (excitation and emission) were measured using a Horiba-Jobin Yvon Fluorolog-3 spectrofluorimeter equipped with a Hamamatsu R928 photomultiplier tube. Spectra were reference corrected for both the excitation source light intensity variation (lamp and grating) and the emission spectral response (detector and grating). All solvents were of spectrophotometric grade. Coumarine 153 and Rubrene were purchased from Acros. Solid-state measurements were performed using a calibrated integrative sphere collecting all the emission ( $2 \pi$ steradians covered with spectralon $\left.{ }^{\circledR}\right)$, model F-3018 from Horiba Jobin Yvon. Because the emission tails extend far in the red emission range, the spectrofluorimeter detector response was checked by recording the emission of known red emitting compounds (4(dimethylamino)-nitrostilbene, tetraphenylporphyrin and rhodamine 6G) and calibrated in consequence.[60] Absolute quantum yields were measured as previously reported.[41]

\subsection{Agregation-induced Emission Measurements}

Stock solutions $(1 \mathrm{mM})$ of the desired compound were prepared in acetone. For each water fraction $\left(f_{w}\right)$, $100 \mathrm{aL}$ of this solution were added in a $2 \mathrm{~mL}$ volumetric flask, followed by the required volume of acetone. Water was then added one-shot to reach $2 \mathrm{~mL}$.

\section{Results and Discussion}

\subsection{Syntheses}




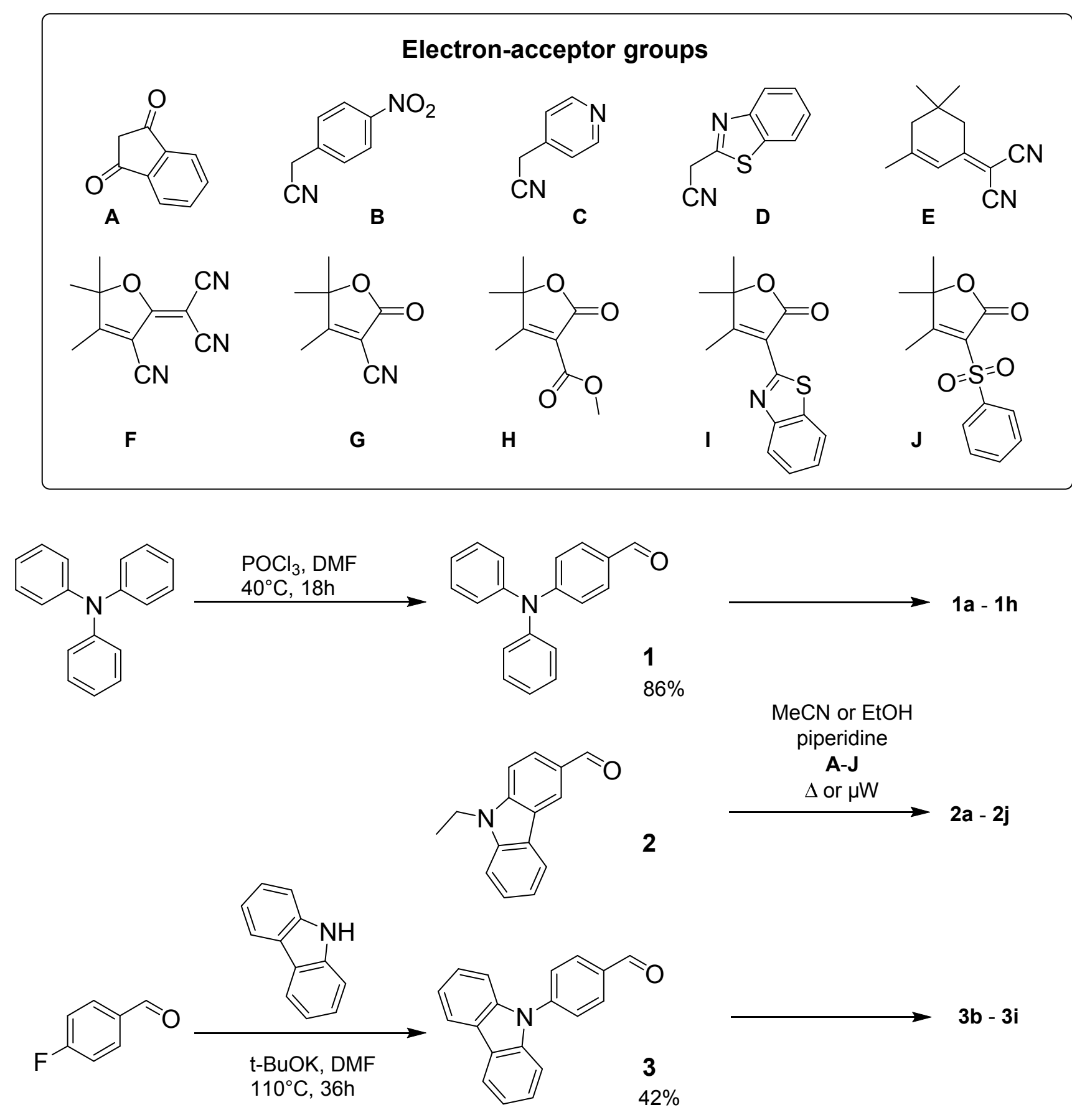

Scheme 1. Structures of activated methylene compounds A-J and synthesis of fluorophores 1a-1h, 2a$\mathbf{2} \mathbf{j}$ and $\mathbf{3 b}-\mathbf{3 i}$.

All compounds $1 \mathbf{a}-\mathbf{3 i}$ were prepared in moderate to good yield by a Knoevenagel condensation between 4-(diphenylamino)benzaldehyde 1, 9-ethyl-9H-carbazole-3-carboxaldehyde $\mathbf{2}$ or 4 -(9Hcarbazol-9-yl)-benzaldehyde $\mathbf{3}$ and the corresponding activated methylene compounds $\mathbf{A}-\mathbf{J}$ in ethanol or acetonitrile in presence of catalytic amount of piperidine (Scheme 1). Conventional or microwave heating are used. Indanedione $\mathbf{A}$ and the 2-substitued acetonitrile derivatives $\mathbf{B}$ - $\mathbf{D}$ are commercially available. Dicyanoisophorone $\mathbf{E}$ was obtained from malonitrile and isophorone according to published procedure, whereas the 2(5H)-furanone ring TCF $\mathbf{F}$ was synthesized from malononitrile and 3-hydroxy3-methyl-butan-2-one 4 with lithium ethoxide in ethanol as a base (Scheme 2).[61] Compounds $\mathbf{G}$ - J 


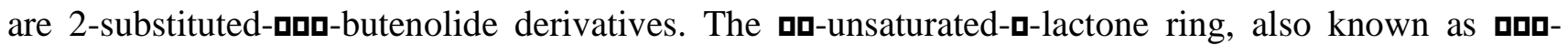
butenolide or $2(5 H)$-furanone, is a structural motif largely found in natural products such as vitamin $\mathrm{C}$, cardenolide alkaloids or annonaceous acetogenin, to cite a few.[62-66] Therefore, substituted 2(5H)furanone rings are interesting building blocks in organic chemistry and their syntheses have been largely investigated.[67-72] 4,5,5-trimethyl-2(5H)-furanone substituted at position $\mathrm{C} 2$ are obtained from 3hydroxy-3-methyl-butan-2-one 4 as shown in Scheme 2. Thus, compounds $\mathbf{G}$ [68] and $\mathbf{H}$ [69] were obtained from 4 and ethyl cyanoacetate or dimethyl malonate, respectively, in presence of a catalytic amount of sodium methoxide in methanol. Subsequent reaction of $\mathbf{G}$ with 2-aminothiophenol in phosphoric acid afforded $\mathbf{I}$ in $66 \%$ yield.[70] $\mathbf{J}$ was obtained in two steps from ethyl 2-bromoacetate by nucleophilic substitution with sodium sulfinate [53] followed by condensation with 4 in presence of sodium methoxide in methanol. The structures of all compounds $\mathbf{1 a}-\mathbf{3 j}$ were unambiguously assigned by ${ }^{1} \mathrm{H}$ and ${ }^{13} \mathrm{C}$ NMR. Although two isomers could potentially arise from the Knoevenagel condensation, the observation of one set of signals in the NMR spectra for the protons connected to the central $\mathrm{C}=\mathrm{C}$ bond, consisting of one singlet or two doublets along with a ${ }^{3} J$ coupling constant of $16 \mathrm{~Hz}$, confirmed the stereoselective formation of the $Z$-isomers only for $\alpha$-cyanostyrene derivatives (i.e. $\mathbf{1 b}-\mathbf{1 d}, \mathbf{2 b}-\mathbf{2 d}$,

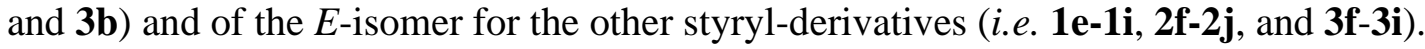

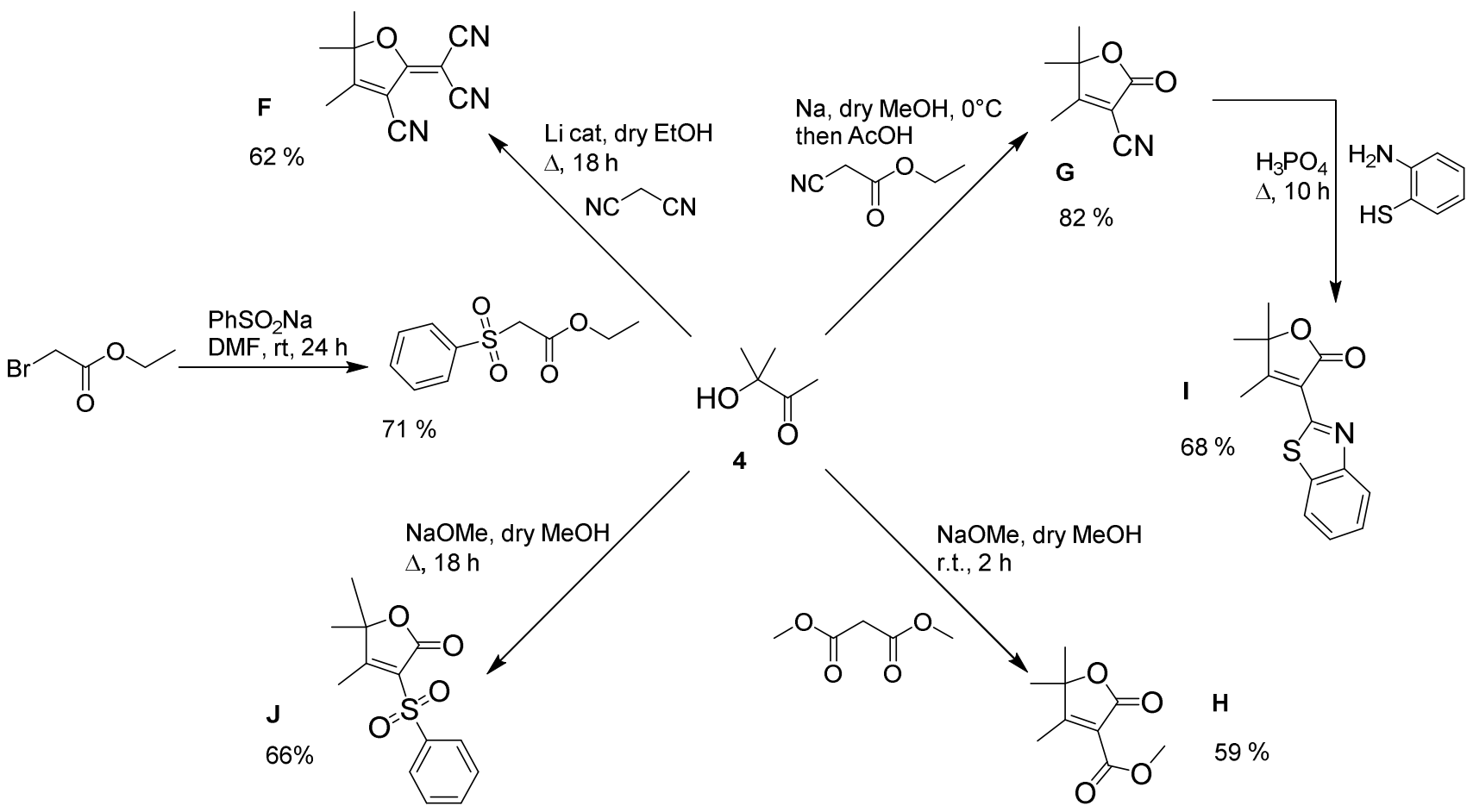

Scheme 2. Synthesis of the electron-accepting groups $\mathbf{F}$ - J from 3-hydroxy-3-methyl-butan-2-one 4.

\subsection{Photophysical Properties in Solution}

The absorption spectra of all compounds were measured in diluted dichloromethane solutions. Relevant photophysical data are reported in Table 1. The spectra displayed in Figures 1 and SI-19 to SI21 showed typical induced charge transfer transitions in the visible with bands positions varying accordingly with the strength of the substituent groups. For a given electron-donating group, increasing the electron-acceptor strength induced a red-shift of the absorption maxima: $\lambda_{\max }$ shifted from $427 \mathrm{~nm}$ for 1c to $565 \mathrm{~nm}$ for 1f bearing the strongest electron-withdrawing group in the 4-(N,Ndiphenylamino)phenyl series, from $389 \mathrm{~nm}$ for $\mathbf{2 c}$ to $510 \mathrm{~nm}$ for $\mathbf{2 f}$ in the 9-ethyl-9H-carbazolyl series, and from $397 \mathrm{~nm}$ for $\mathbf{3 h}$ to $481 \mathrm{~nm}$ for $\mathbf{3 f}$ in the 4-(9H-carbazol-9-yl) series. In all cases, the strongest electro-accepting group $\mathbf{F}$ led to the most red-shifted absorption. Upon excitation in the main absorption band only a weak fluorescence was observed for most compounds likely because of radiationless decay 
from TICT excited states (Figures 1, SI-22 to SI-24). Only compounds $1 \mathbf{1 a}, \mathbf{1 e}, \mathbf{1 g}, \mathbf{1 h}, \mathbf{1 i}, \mathbf{3 g}$, and $\mathbf{3 i}$ displayed significant emission $(\Phi>2 \%)$, up to $20 \%$ at $689 \mathrm{~nm}$ for 1e. This low emission could be anticipated especially for molecules built on the $\alpha$-cyano-stilbene motif $(\mathbf{1 b}, \mathbf{1 c}, \mathbf{1 d}, \mathbf{2 b}, \mathbf{2 c}, \mathbf{2 d}, \mathbf{3 b})$ that undergo very efficient non-radiative de-excitations via twisted conformations of chromophores in solution, torsional movements around the central $\mathrm{C}=\mathrm{C}$ double bond [73-76] or cis-trans isomerization.[77] Emissions are characterized by large Stokes' shifts ranging from $1328 \mathrm{~cm}^{-1}$ for 2a to $9462 \mathrm{~cm}^{-1}$ for $\mathbf{3 b}$, typical of an excited state charge transfer and of high difference between dipole moments in ground and excited states. Even though this is not the object of the present article, this latter parameter is important in view of obtaining large biphotonic fluorescence efficiencies, a plus for potential biological applications. On the other hand, the emission maxima ranged from $502 \mathrm{~nm}(\mathbf{2 d})$ to over $700 \mathrm{~nm}$ for the most red-shifted compounds $\mathbf{1 b}(717 \mathrm{~nm})$, 1f $(746 \mathrm{~nm})$ and $\mathbf{3 f}(712 \mathrm{~nm})$. Given the importance of NIR emission for deep in vivo imaging,[78] such long emission wavelengths are interesting in spite of the low emission quantum yields, particularly for such small molecular weight molecules (molecular weight below $460 \mathrm{~g} \cdot \mathrm{mol}^{-1}$ ).

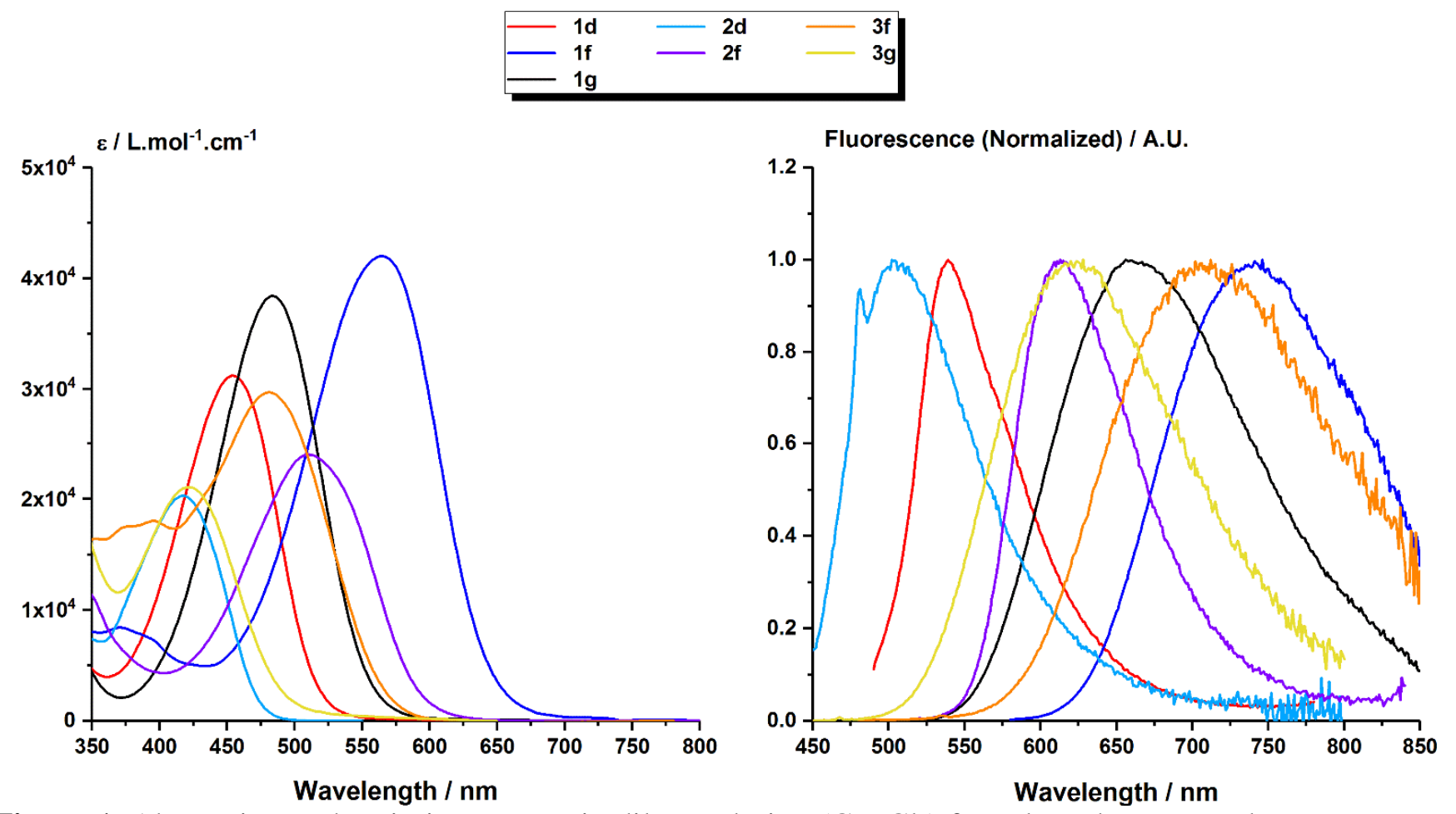

Figure 1. Absorption and emission spectra in dilute solution $\left(\mathrm{CH}_{2} \mathrm{Cl}_{2}\right)$ for selected compounds. 
Table 1. Spectroscopic data in dilute solution $\left(\mathrm{CH}_{2} \mathrm{Cl}_{2}\right)$ and in the crystal state for all compounds.

\begin{tabular}{|c|c|c|c|c|c|c|}
\hline \multirow[b]{2}{*}{ Compound } & \multicolumn{4}{|c|}{ Solution in $\mathrm{CH}_{2} \mathrm{Cl}_{2}$} & \multicolumn{2}{|c|}{ Crystal state $^{[\mathrm{c}]}$} \\
\hline & $\begin{array}{l}\lambda_{\text {abs }} / \mathrm{nm} \\
\left(\varepsilon / \mathrm{L} \cdot \mathrm{mol}^{-1} \cdot \mathrm{cm}^{-1}\right)\end{array}$ & $\lambda_{\mathrm{em}} / \mathrm{nm}$ & $\Phi^{[a]} / \%$ & $\Delta v / \mathrm{cm}^{-1[\mathrm{~b}]}$ & $\lambda_{\mathrm{em}} / \mathrm{nm}$ & $\Phi^{[\mathrm{d}]} / \%$ \\
\hline $\mathbf{1 a}$ & $485(37800)$ & 644 & 2 & 5091 & 618 & 11 \\
\hline $1 b$ & $456(28700)$ & 717 & $<1$ & 7983 & 669 & 4 \\
\hline 1c & $427(29700)$ & 572 & $<1$ & 5937 & 640 & 23 \\
\hline 1d & $454(31200)$ & 539 & $<1$ & 3474 & 647 & 18 \\
\hline 1e & $490(23600)$ & 689 & 20 & 5894 & n.a. & n.a. \\
\hline 1f & $565(42000)$ & 746 & 1 & 4294 & n.a. & n.a. \\
\hline $1 g$ & $484(38400)$ & 656 & 6 & 5417 & 645 & 9 \\
\hline $1 \mathrm{~h}$ & 455 (34900) & 636 & 3 & 6255 & 640 & 10 \\
\hline $\mathbf{1 i}$ & $455(34900)$ & 633 & 7 & 5054 & 673 & 19 \\
\hline $2 \mathbf{a}$ & $453(44800)$ & 482 & $<1$ & 1328 & 599 & 18 \\
\hline $2 \mathbf{b}$ & $405(21300)$ & 616 & $<1$ & 8458 & 603 & 28 \\
\hline $2 c$ & 389 (25000) & 471 & $<1$ & 4476 & 551 & 10 \\
\hline 2d & $417(20300)$ & 502 & $<1$ & 4060 & 619 & 11 \\
\hline $2 f$ & $510(24000)$ & 613 & 2 & 3295 & 768 & 4 \\
\hline $2 h$ & $413(22590)$ & 576 & $<1$ & 6852 & 583 & 8 \\
\hline $2 \mathbf{i}$ & $452(22600)$ & 533 & $<1$ & 3362 & 614 & 34 \\
\hline $2 \mathrm{j}$ & $439(26100)$ & 546 & $<1$ & 4464 & 598 & 33 \\
\hline $3 b$ & $404(21110)$ & 654 & $<1$ & 9462 & 596 & 11 \\
\hline $3 f$ & $481(29700)$ & 712 & $<1$ & 6745 & 735 & 11 \\
\hline $3 g$ & $421(21070)$ & 628 & 6 & 7829 & 622 & 24 \\
\hline $3 \mathbf{h}$ & $397(23200)$ & 593 & 2 & 8326 & 534 & 24 \\
\hline $3 \mathbf{i}$ & $417(20630)$ & 583 & 4 & 6828 & 603 & 12 \\
\hline
\end{tabular}

${ }^{\text {[a] }}$ Using Rubrene in methanol as reference $(\Phi=27 \%)$ or Coumarine 153 in methanol $(\Phi=54 \%) .{ }^{\text {[b] }}$ Stokes' shifts. ${ }^{[\mathrm{c}]}$ In crystalline powder. ${ }^{[\mathrm{d}]}$ Using a calibrated integrated sphere.

\subsection{Aggregation-Induced Emission}

Aggregation-induced emission (AIE) fluorophores typically showed an increase in fluorescence intensity from the non-fluorescent or weakly fluorescent molecule in dilute organic solution to the strongly fluorescent suspension of nanoparticles that formed when water is added to the solvent. Good AIE properties may be expected from solids that are strongly emissive in their crystalline state, although the emission wavelength and efficiency can be different, as we already noticed.[41]

The AIE behavior of some compounds was studied by recording the emission spectra obtained from acetone/water mixtures of fluorophores at the same concentration $(10 \mu \mathrm{M})$, but with different volume fractions of water $\left(f_{w}\right)$. The fluorescence of push-pull dipolar D- $\pi$-A compounds is usually weaker in polar solvents because enhanced electrostatic interactions with the chromophore strongly polarized ICT excited state. So as expected, the emission in pure acetone $\left(f_{w}=0\right)$ is significantly decreased in comparison with dichloromethane. The emission is further quenched when $f_{w}$ was increased from 0 to $50-60 \%$ where the solvating power of the solvent mixture is still sufficient to dissolve the compounds and prevent aggregation, due to the higher polarity of the solvent system. Then, when $f_{w}$ was gradually increased from $60 \%$ volume fractions of water to $95 \%$, the products start to aggregate and form nanoparticles, whereas the fluorescence intensity increased considerably. Remarkably, all the compounds studied showed AIE, but noticeable differences could be evidenced as illustrated in Figure 2 and SI-25 to SI-35. On the other hand, the emission maxima of nanoparticles underwent a blue-shift with respect to pure acetone solution, for all compounds except 1d (Fig. SI-28) and 2a (Fig. SI-33). The maximum emission intensity was reached between 80 and $95 \%$ water volume fractions depending on the compound. In most cases, a decrease of the fluorescence intensity can be seen at higher $f_{w}$. The overall increase of fluorescence between solutions and nanoparticles $\left(\alpha_{A I E}\right)$ was of the order of $\alpha_{\text {AIE }}=3$ -

20 but could reach values higher than 100 for some compounds. Remarkable increases of more than 348 and 960 were obtained for $\mathbf{3 g}$ and $\mathbf{3 f}$ respectively, the most interesting compounds. This is reflected by the quantum yield values of nanoparticles, generally comprised between $2 \%$ and $8 \%$, except for $3 \mathbf{f}$ for 
which a noteworthy $20 \%$ quantum yield value at $678 \mathrm{~nm}$ was obtained, to be compared with the values measured in dichloromethane solution. It has to be noted that $\mathbf{1 i}$ and $\mathbf{3 g}$ are as fluorescent in their dissolved form in dichloromethane than in nanoparticles. Note that these results are in perfect agreement with those previously reported for compound $\mathbf{2 a}\left(\lambda_{\mathrm{em}}=630 \mathrm{~nm}\right.$ for a $15 \%$ quantum yield), although the initial solvent used was not the same (THF instead of acetone here).[39]

Table 2. Optical properties in nanoparticles (acetone/water mixture).

\begin{tabular}{|c|c|c|c|c|}
\hline \multirow{2}{*}{ Compound } & \multicolumn{4}{|c|}{ Nanoparticles (acetone/water) ${ }^{\text {[a] }}$} \\
\hline & $\lambda_{\mathrm{abs}} / \mathrm{nm}$ & $\lambda_{\mathrm{em}} / \mathrm{nm}$ & $\alpha_{\mathrm{AIE}}$ & $\Phi / \%$ \\
\hline 1a & 493 & 635 & 106 & 8 \\
\hline $\mathbf{1 b}$ & 467 & 664 & 3 & $<1$ \\
\hline 1c & 437 & 595 & 20 & 2 \\
\hline 1d & 450 & 649 & 20 & 4 \\
\hline 1e & 490 & 698 & 9 & 3 \\
\hline 1f & 544 & 749 & 17 & $<1$ \\
\hline $1 \mathrm{~g}$ & 489 & 659 & 136 & 6 \\
\hline $\mathbf{1 i}$ & 465 & 625 & 9 & 4 \\
\hline 1h & 493 & 636 & 59 & 5 \\
\hline $\mathbf{2 a}$ & $468(460)^{[b]}$ & $630(630)^{[b]}$ & 105 & n.d $(15 \%)^{[b]}$ \\
\hline 3f & 580 & 678 & 969 & 20 \\
\hline $3 g$ & 425 & 616 & 348 & 8 \\
\hline $3 \mathbf{i}$ & 420 & 590 & 17 & 6 \\
\hline
\end{tabular}

${ }^{\text {[a] }}$ In acetone/water at $f_{w}$ giving the maximum emission. ${ }^{[b]}$ according to [39]. THF solution was used instead of acetone. 
a)

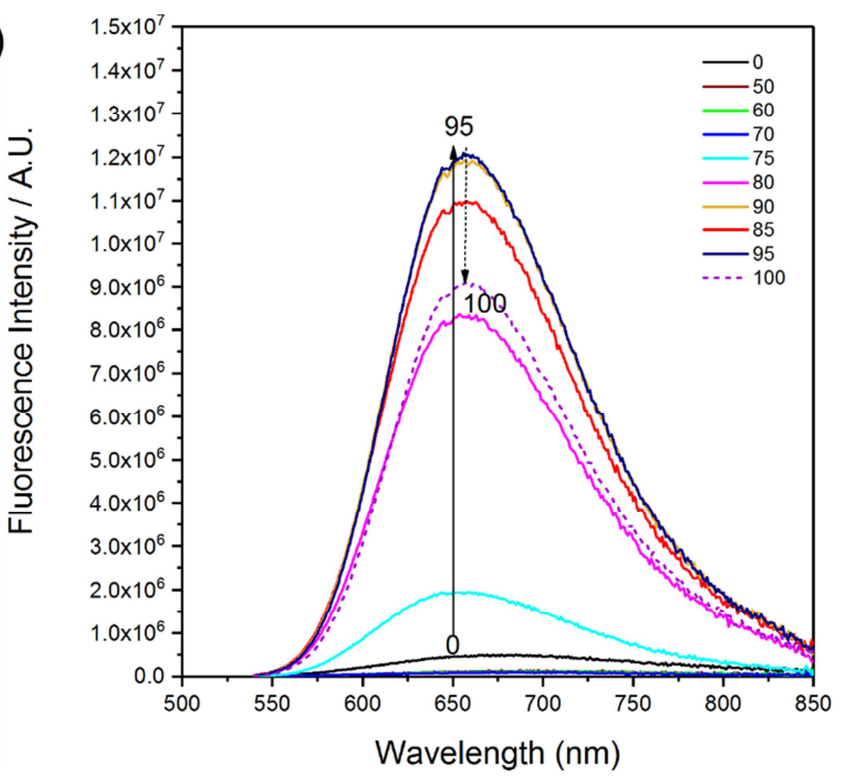

b)

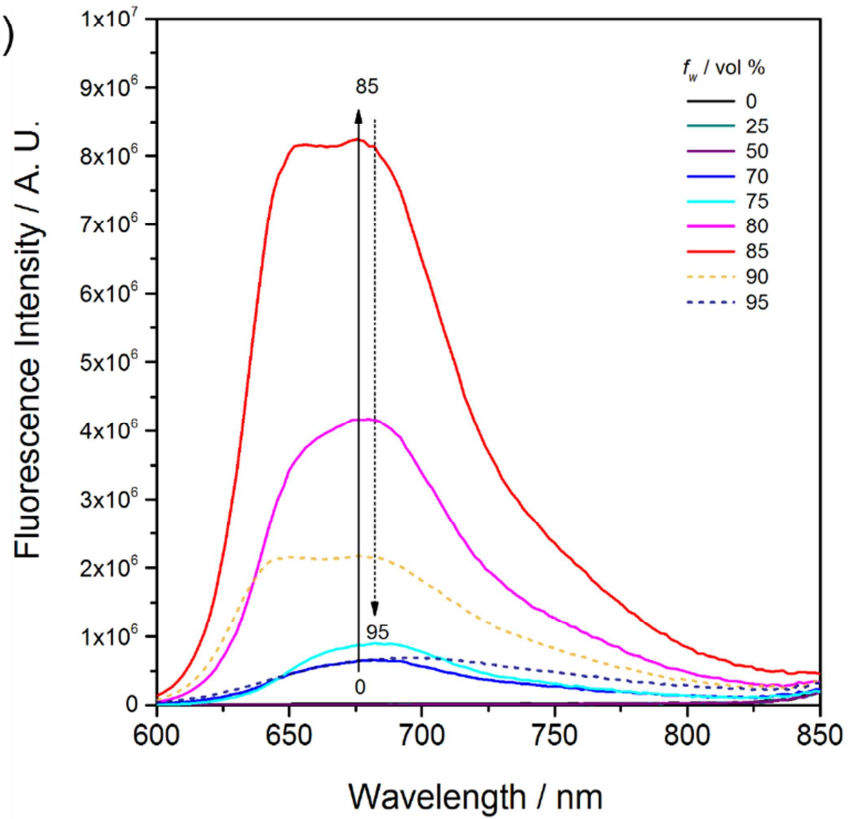

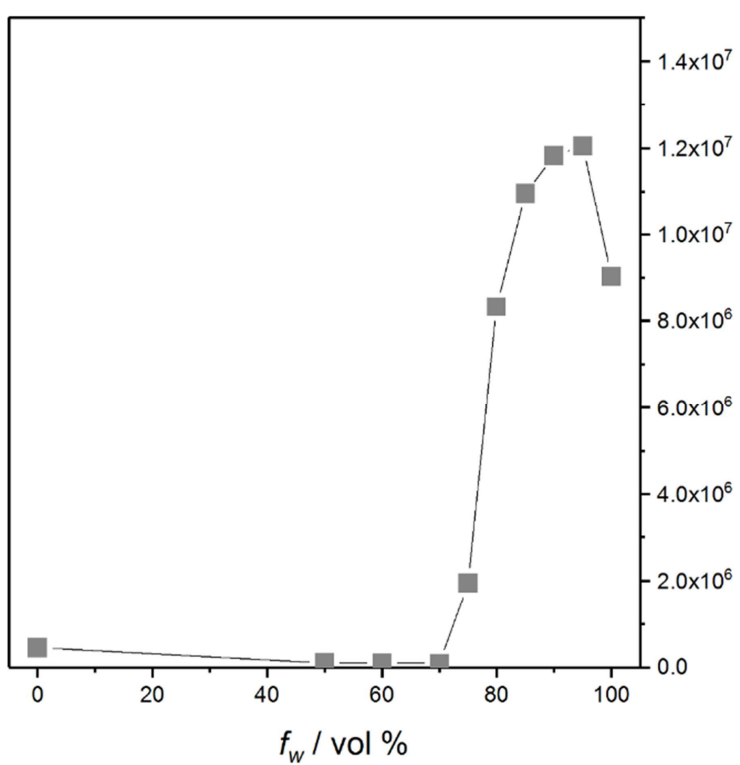

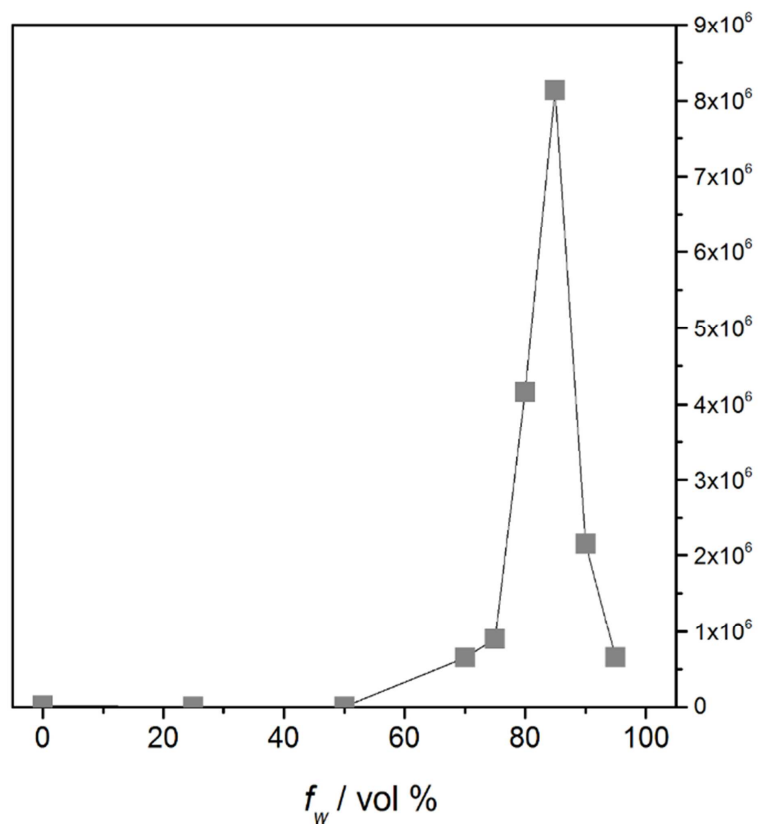

Figure 2. Emission spectra in acetone/water mixture of different water fraction $\left(f_{w}\right)$, and change of the peak intensity with $f_{w}$ for $\mathbf{1 g}(\mathrm{a})$ and $\mathbf{3 f}(\mathrm{b}), \mathrm{c}=10 \mu \mathrm{M}$.

\subsection{Emission in the Crystal State}

Encouraged by the observations made in nanoparticles, we then turned our attention to the study of the emission in the crystal-state (micrometric powders). All compounds with the exception of $\mathbf{1 e}$ and $\mathbf{1 f}$ displayed an intense orange-red emission in their crystalline form under illumination by a handled UV lamp at $365 \mathrm{~nm}$, visible to the naked eye. Emission and excitation spectra as well as fluorescence quantum yields were therefore measured using a calibrated integrating sphere. Emission spectra are given in Figure 3 and in the Supporting Information (Figures SI-36 to SI-38) and data summarized in Table 1. In the crystal state, the emission was, in most cases, significantly red-shifted relative to the emission in solution in dichloromethane, 20 to $60 \mathrm{~nm}$ and up to $115 \mathrm{~nm}$ for compound $2 \mathbf{d}$ displaying an emission maximum at $620 \mathrm{~nm}$. Compounds $\mathbf{1 a}, \mathbf{1 b}, \mathbf{2 b}$ and $\mathbf{3 b}$, for which a small blue shift (20 to $45 \mathrm{~nm}$ ) was observed, were of notable exception. However, as expected, the strongest electronaccepting group $(T C F)$ induces the maximum red-shift (compounds $2 \mathbf{f}$ and $3 \mathbf{f}$ having emission above $700 \mathrm{~nm}$ ). The emission quantum yields were considerably increased though, reaching $34 \%$ at $614 \mathrm{~nm}$ for the most emissive compound $\mathbf{2 i}$ and $11 \%$ at $735 \mathrm{~nm}$ for $\mathbf{3 f}$, which is remarkable for such small 
molecule.

Fluorescence (Normalized) / A.U.

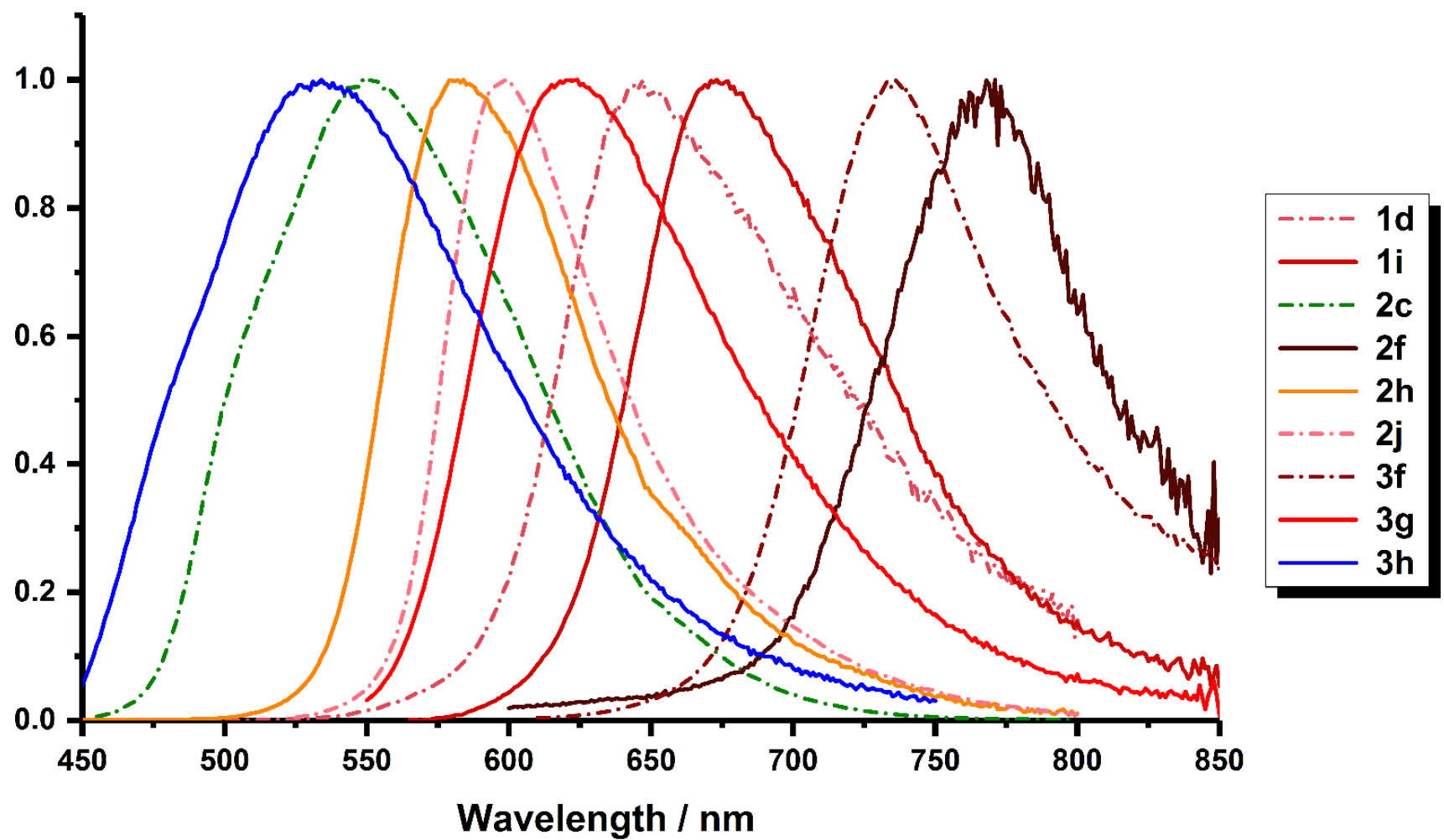

Figure 3. Crystal-state fluorescence spectra of selected compounds showing the tuning of emission wavelengths spanning the entire visible range.

\subsection{Crystal structures}

Although no study has directly linked the solid-state fluorescence properties to the crystalline structure, several have nevertheless pointed out that emission in solid may depend on the arrangements of the molecules in the crystal.[27, 79] Thus, on dipolar structure similar to those presented here, we have been able to show that certain patterns like ladder-like, brickwork or herringbone patterns could be observed in the crystal packing of emissive compounds, in which the molecules are slipped away with respect from one another preventing close packing.[40, 41] Conversely, the same studies associated the lack of crystal-state emission to the presence of closely packed face-to-face $H$-type dimers in the crystal structure. This was perfectly illustrated by the example of compounds $\mathbf{1 e}$, for which two molecules lie at short distance with respect to one another (3.587 $\AA$ ) with strong $\pi$ - $\pi$ interactions (Figure 4).[40]

So knowledge of the crystal-state structure and the associated molecular packing could nevertheless help understanding the origin of the solid-state emission. To that end single crystals were grown by slow diffusion of a non-solvent (diisopropylether) in concentrated chloroform or dichloromethane solutions. Thus, we obtained sub-millimeter size crystal suitable for X-ray diffraction for nine new compounds over twenty-two synthesized and the structures were resolved (compounds $\mathbf{1 a}, \mathbf{1 b}, \mathbf{1 d}, \mathbf{1 g}, \mathbf{1 i}, \mathbf{2 b}, \mathbf{2 j}, \mathbf{2} \mathbf{i}$ and 3f). Crystallographic data and refinement parameters are given in Table 3, SI-2 and SI-3, whereas basic structural parameters, selected distances and dihedral angles are compiled in SI (Table SI-1) together with atom numbering schemes with the angles and distance definitions (Figure SI-1). ORTEP views with 50\% probability are shown in Figures SI-2 to SI-10. 


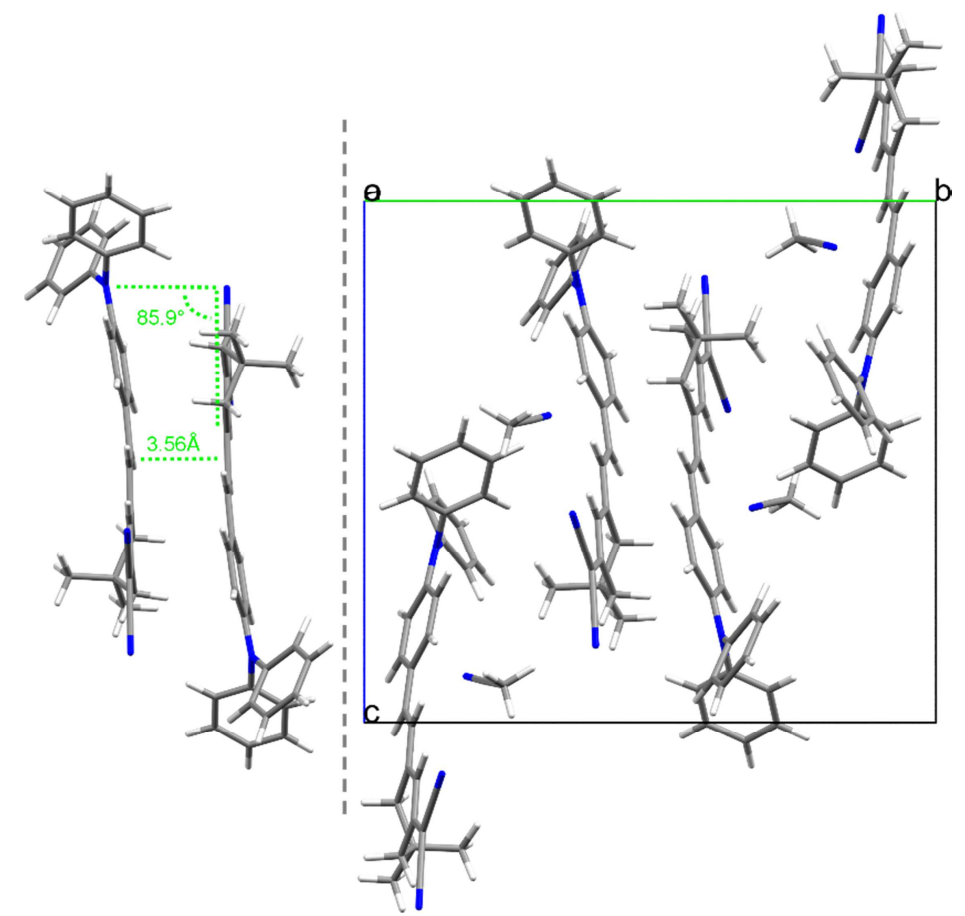

Figure 4. Compound 1e, stacked dimer (left) and crystal packing view along the crystallographic $a$ axis (right).

Compounds $\mathbf{1 a}$ and $\mathbf{1 d}$ crystallize in the $P \overline{1}$ triclinic space group. Compounds $\mathbf{1 b}, \mathbf{1 e}, \mathbf{1 g}, \mathbf{1 i}, \mathbf{2} \mathbf{i}$ and 2j crystallize in the $P 21 / \mathrm{n}$ or $P 2_{1} / \mathrm{c}$ monoclinic space groups. $2 \mathbf{b}$ and $\mathbf{3 f}$ crystallize in the noncentrosymmetric orthorhombic $F \mathrm{dd} 2$ and monoclinic $P 2_{1}$ space groups respectively. The asymmetric units are composed of one molecule, except for $\mathbf{1 d}$ and $\mathbf{1 g}$ where two crystallographically independent molecules are present. As already observed for related dipolar molecules [40, 44, 80-82] or $N$, $N$-diethyl analogues, $[40,83]$ the molecular structures are very similar for all compounds with an almost planar $\pi$ conjugated system indicating a full conjugation between the donor and the acceptor end. Only the acceptor end of the dipole is slightly twisted with a dihedral angle measured between the main plane and the electron-accepting ring of $31.5^{\circ}$ for the most distorted structures $\mathbf{1 g}$ and $\mathbf{2 j}$, which is bend with a $14.4^{\circ}$ angle between the $2(5 H)$-furanone ring mean plane and the carbazole ring mean plane. As expected the two phenyl groups on the $\mathrm{N}$-donor atom are not coplanar with the main molecular plane for steric reasons but adopt a propeller type conformation. $[25,33]$ Such a conformation is not possible for the carbazole ring in compound $\mathbf{3 f}$ due to the additional $\mathrm{C}-\mathrm{C}$ bond, but steric hindrance hampers the planarity and the carbazole ring is out of the main plane twisted by $35^{\circ}$. The carbazole rings of compounds $\mathbf{2 b}, \mathbf{2} \mathbf{i}$ and $\mathbf{2} \mathbf{j}$ on the other hand are fully planar and form the molecular plane as expected.[44] For compound $\mathbf{2} \mathbf{j}$ finally, the phenyl ring of the phenyl sulfonyl group is almost perpendicular to the molecular plane $\left(128^{\circ}\right)$. Interestingly, for compound $\mathbf{1 a}$ two aromatic C-C bonds of the donor para-phenylene ring, C14-C15 [1.378 (3)] and C30-C31 [1.379 (3) A], are slightly shorter than the other four aromatic bonds [1.403 (4)-1.407 (3) $\AA$ ] suggesting a para-quinoid character of the donor ring. Finally, whereas s-trans of the electron accepting group relative to the central double bond $\left(b_{1}\right)$ was observed for most compounds, the two crystallographically independent molecules present in the unit cell of $1 \mathrm{~g}$ adopt different conformations, one being s-trans the other one s-cis (molecule A) and more twisted.

Here again, representative $J$-aggregates[84] in the form of ladder-like/brickwork (1a, 1d, 2i, 3f) patterns, herringbone $(\mathbf{1 b}, \mathbf{1 g}, \mathbf{1 i}, \mathbf{2} \mathbf{b}$,) pattern, or both $(\mathbf{2} \mathbf{j})$, can be seen in the crystal packing of the fluorescent solids. Ladder-like and brickwork patterns are created by long chains of dipole lying one next to the other (1a, 1d, $\mathbf{2 i}, \mathbf{2 j}$ ) or following each other (3f), that are stacked on top of each other. Two neighboring chains defining a common plane may point in the same direction $(\mathbf{1 a}, \mathbf{2 j}, \mathbf{3 f})$ or in the 
opposite direction (1d, $\mathbf{2 i}$ ), but are always shifted with respect to one another. Herringbone patterns, on the other hand, are built by broken lines of molecules following each other. In all cases, molecules are slipped away (1a, $\mathbf{1 b}, \mathbf{1 d}, \mathbf{1 g}, \mathbf{2 b}, \mathbf{2} \mathbf{j}, \mathbf{3 f})$ and/or tilted $(\mathbf{2 b}, \mathbf{2} \mathbf{i}, \mathbf{2} \mathbf{j})$ with respect from one another preventing close packing. The different patterns observed are schematized in Figures SI-11 to SI-18.
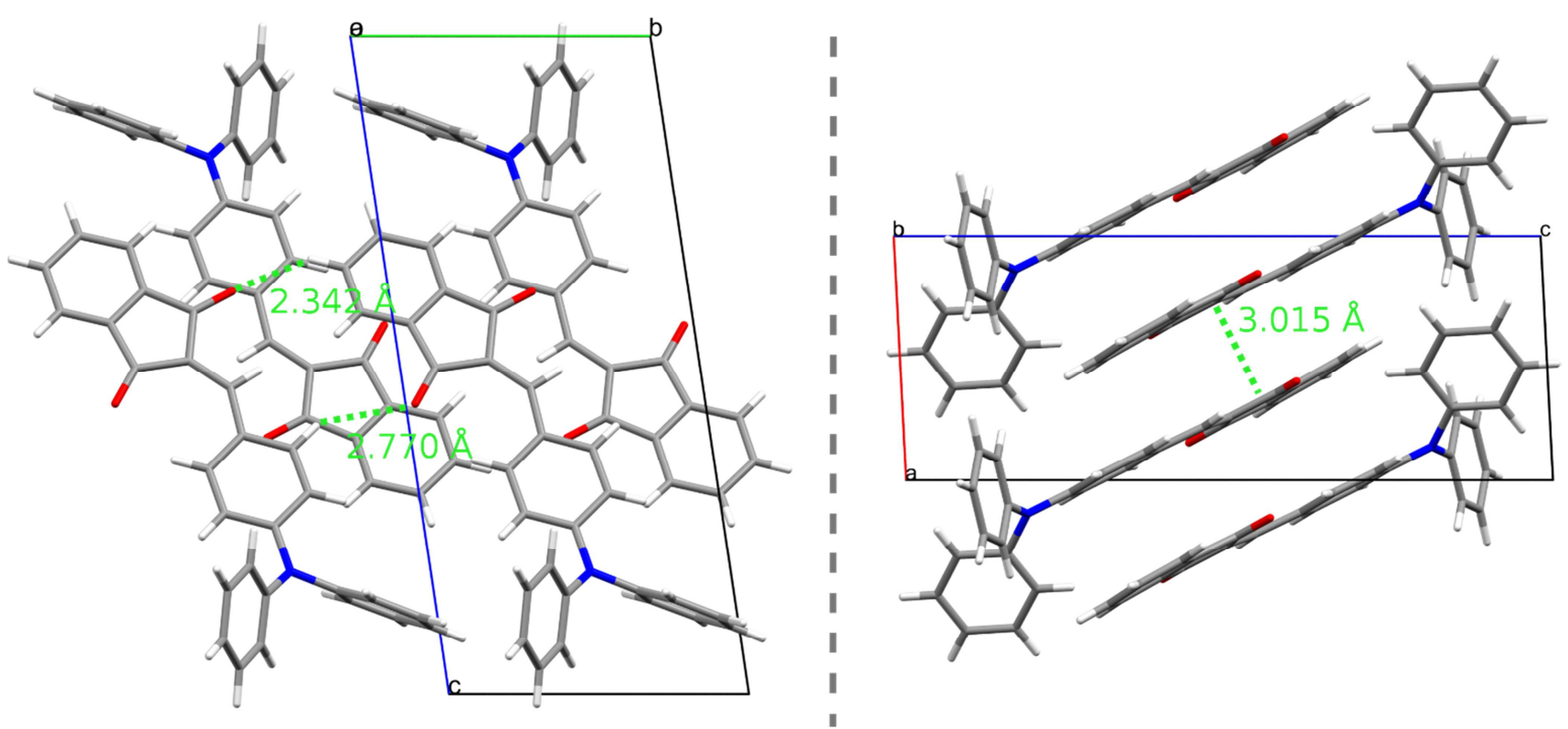

Figure 5. Crystal packing of 1a view along the crystallographic $a$ axis highlighting the brickwork pattern created by the H-bonds (left) and along the $b$ axis showing the ladder-like pattern (right).

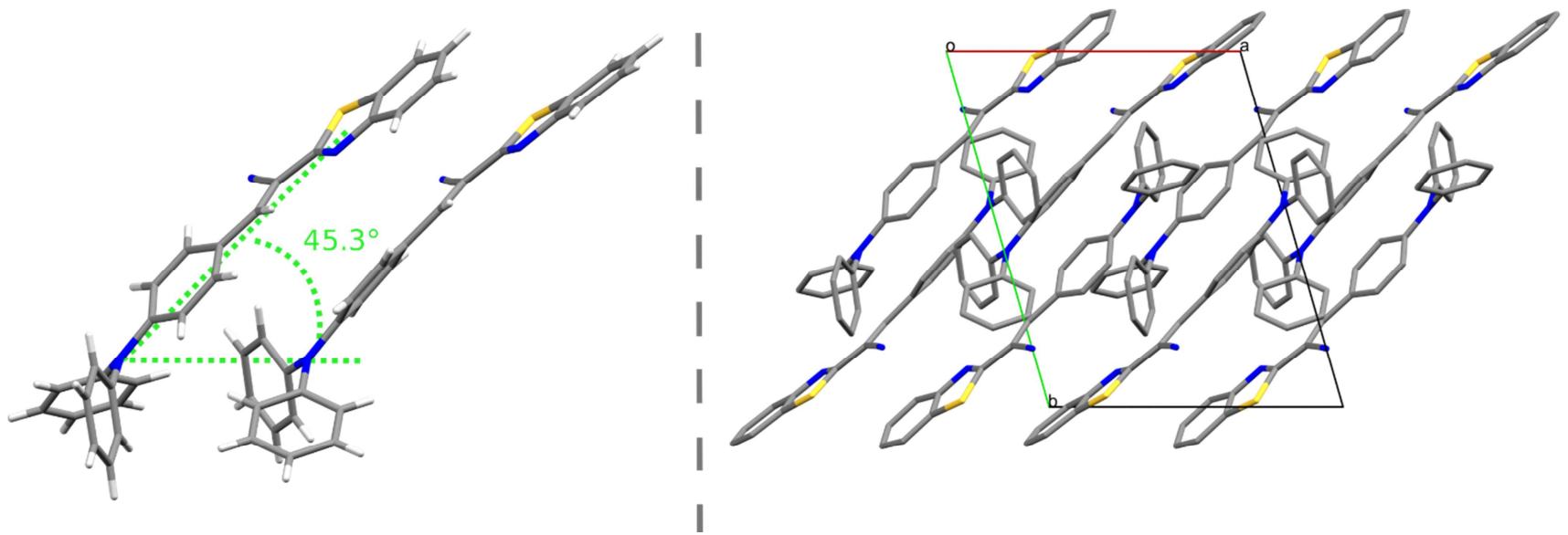

Figure 6. Crystal packing of 1d showing the staircase arrangement of the two independent molecules (left) and repetition of the dimer motif along the c direction creating the ladder-like pattern (right), hydrogen atoms were removed for clarity.

So, the ladder-like crystal packing of 1a, shown in Figure 5-right, is composed of juxtaposed dipoles all parallel to each other and interacting through long H-bonds $(2.342 \AA$ and $2.770 \AA)$ between the $\mathrm{C}=\mathrm{O}$ groups and two aromatic $\mathrm{C}-\mathrm{H}$ (Figure 5-left). This creates ribbons and a brickwork pattern growing in the $b$ direction. Two neighboring bands, in which the dipoles are opposed and slightly slipped away longitudinally and transversally from each other, lies at a distance of $3.07 \AA$ to each other. In the crystal packing of 1d, the two independent molecules of the unit cells are oriented in the same direction, slipped away by $3.9 \AA$ with a slip angle of almost $45^{\circ}$, archetype of staircase $J$-aggregate (Figure 6-left and Figure SI-11). Repetition of the dimeric motif along the $a$ direction form a sheet parallel to the (001) plane in which all molecules are oriented in the same direction. Packing is formed by inversion of the sheet and translation along the $c$ direction (Figure 6-right). 


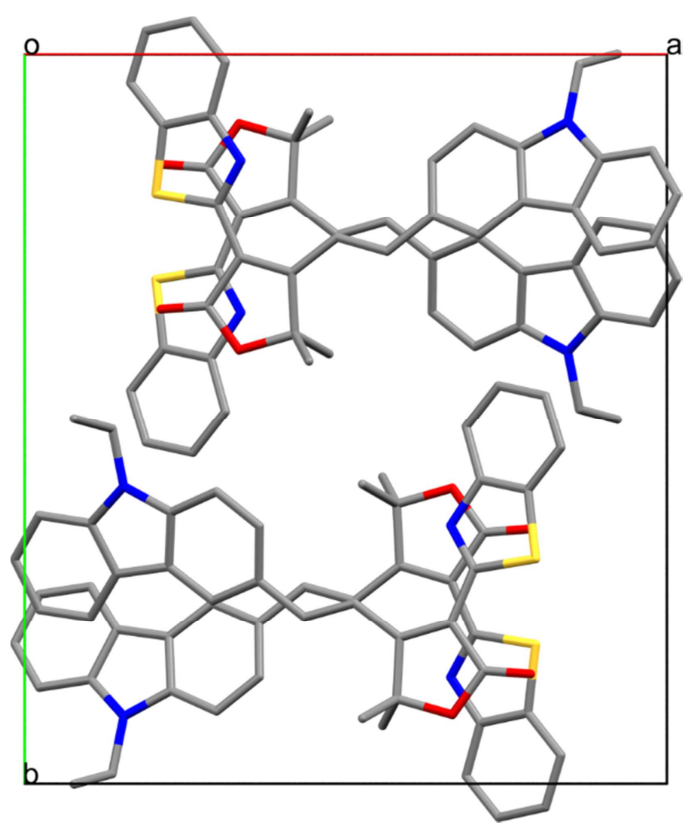

Figure 7. Crystal packing of $\mathbf{2 i}$ viewed along the crystallographic $c$ axis showing the classical brickwork pattern created by two juxtaposed head-to-tail slightly slipped away, hydrogen atoms were omitted for clarity.

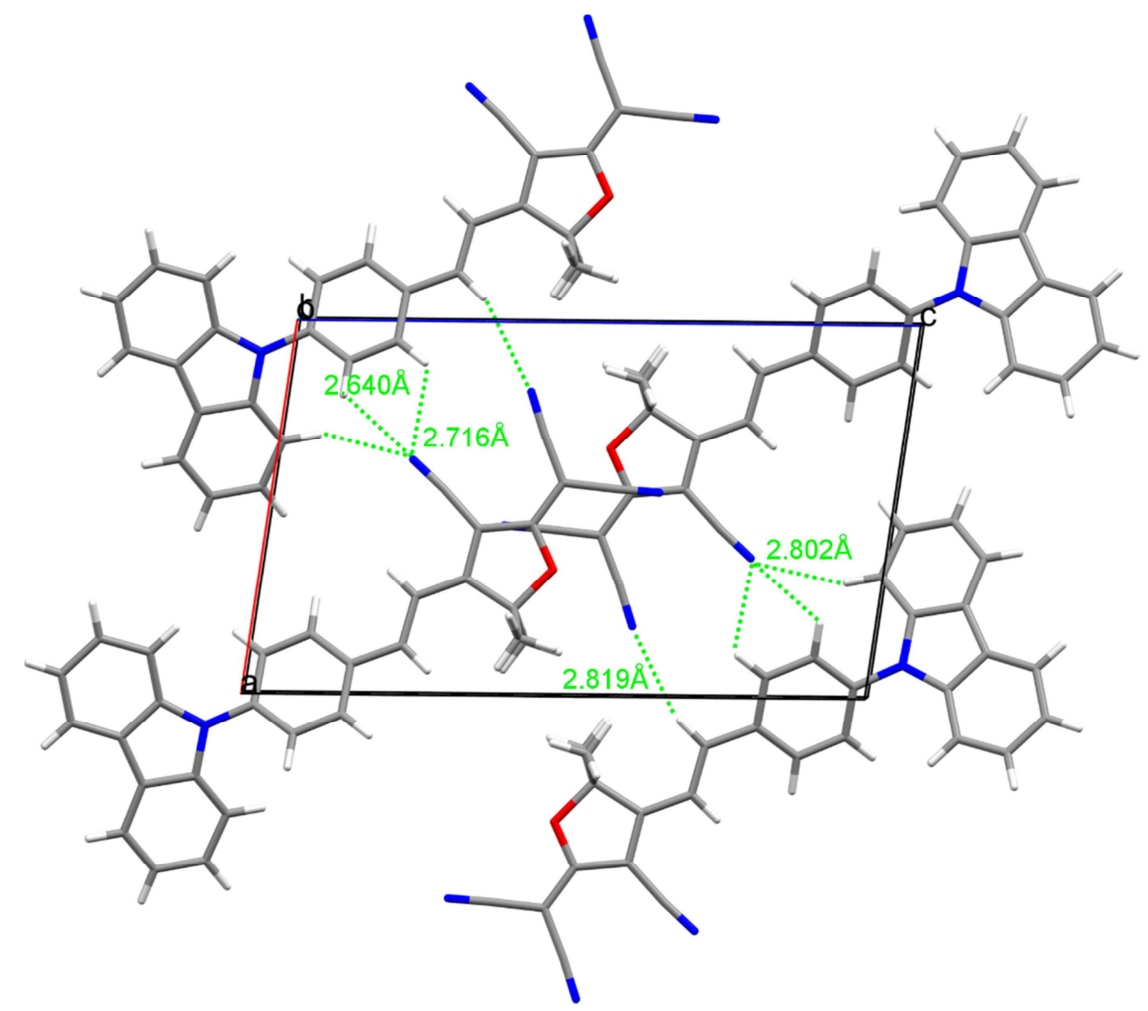

Figure 8. Crystal packing of $\mathbf{3 f}$ viewed along the crystallographic $b$ axis showing the brickwork pattern.

The crystal packing of $\mathbf{2 i}$ (Figure 7) and $\mathbf{3 f}$ (Figure 8) are best described in terms of infinite chains of in-line dipoles, slightly wavy in the case of $\mathbf{2 i}$. By translation along the axis perpendicular to the molecular one but in the molecular plane, parallel planes are formed creating the brickwork patterns, somewhat distorted in the case of $\mathbf{2 i}$. Two planes stand $c a 3.5 \AA$ (3f) and $3.7 \AA$ (2i) apart (Figure SI-13 and SI-15). Within a plane, two neighboring chains are either parallel (3f) or anti parallel (2i) to each 
other. In the case of $\mathbf{3 f}$, two neighboring chains are connected through multiple weak H-bonds between $\mathrm{C}-\mathrm{H}$ of the one molecule and the $-\mathrm{C} \equiv \mathrm{N}$ group of the adjacent one. For $\mathbf{2 i}$, small inclination of two adjacent molecules with respect to each other draw waves propagating in the $a$ and $c$ directions. Note that among the compound emitting in the crystal state and nanoparticles, $\mathbf{3 f}$ displayed the most redshifted emission in solid and AIE.

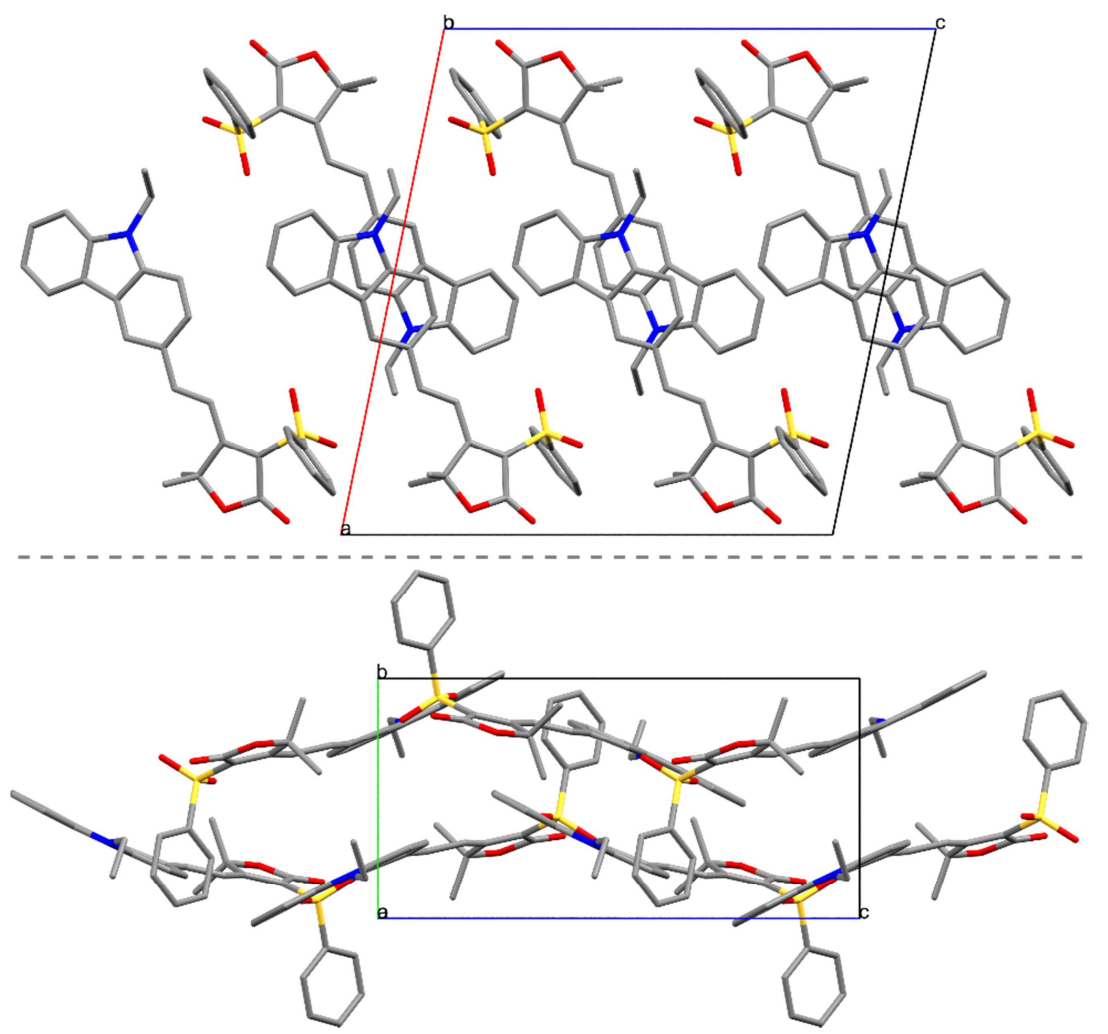

Figure 9. Crystal packing of $\mathbf{2} \mathbf{j}$ viewed along $a$ crystallographic showing the ladder-like pattern resulting from the repetition of head-to-tail molecules (top) and along $b$ crystallographic axis showing the inclination of the molecules and the herringbone pattern (bottom). Note that Hydrogen atoms are omitted for clarity.

Compound $\mathbf{2 j}$ crystal packing combines ladder-like and herringbone patterns. Stacks of head-to-tail molecules, $2.481 \AA$ apart and considerably slipped away longitudinally by ca $5.8 \AA$, overlapping only at the level of the carbazole group, build the ladder growing along the $b$ direction (Figure 9-top and Figure SI-14). On the other hand, two adjacent molecules, in which the benzenesulfonyl groups are placed alternatively above and below the mean plane of the molecular planes, are inclined with respect to each other forming an angle of $51^{\circ}$, creating an undulation along the $c$ direction (Figure 9-bottom), sort of herringbone pattern. Note that $\mathbf{2} \mathbf{j}$ (together with $\mathbf{2} \mathbf{i}$ ) present the highest emission quantum yield (respectively $33 \%$ and $34 \%$ at $598 \mathrm{~nm}$ and $614 \mathrm{~nm}$ respectively). 


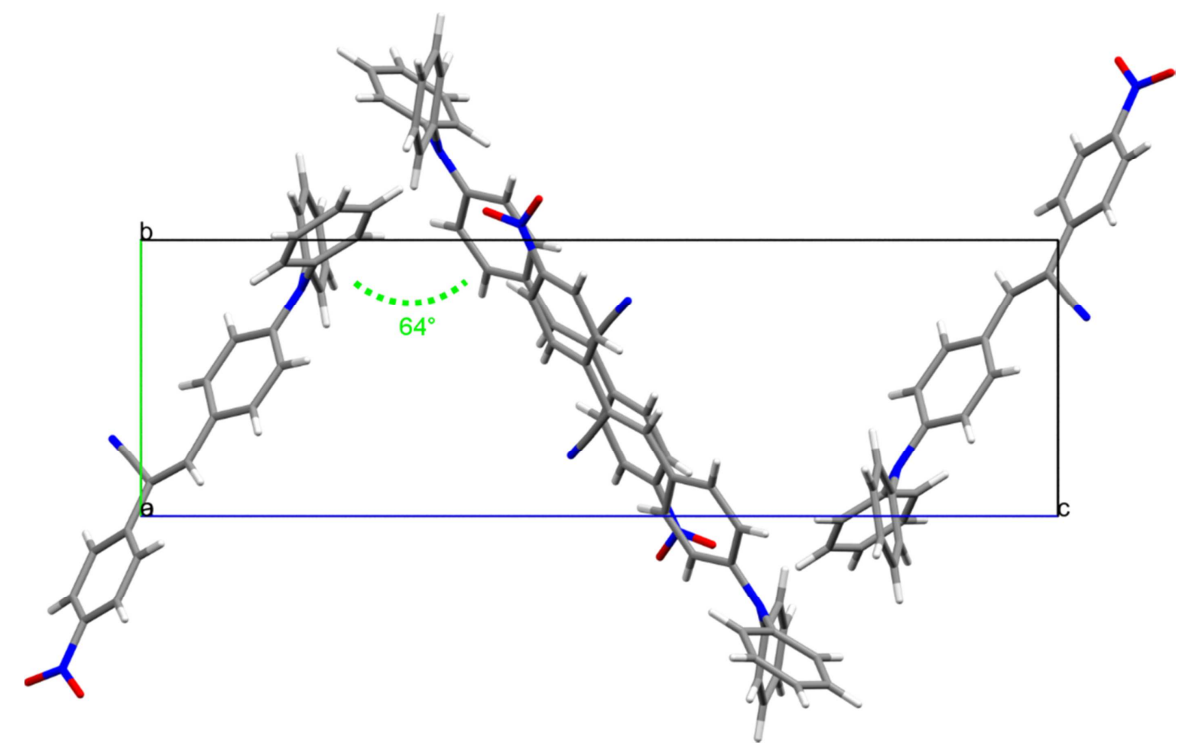

Figure 10. Crystal packing of $\mathbf{1 b}$ viewed along $a$ crystallographic axis showing the well-defined herringbone pattern.
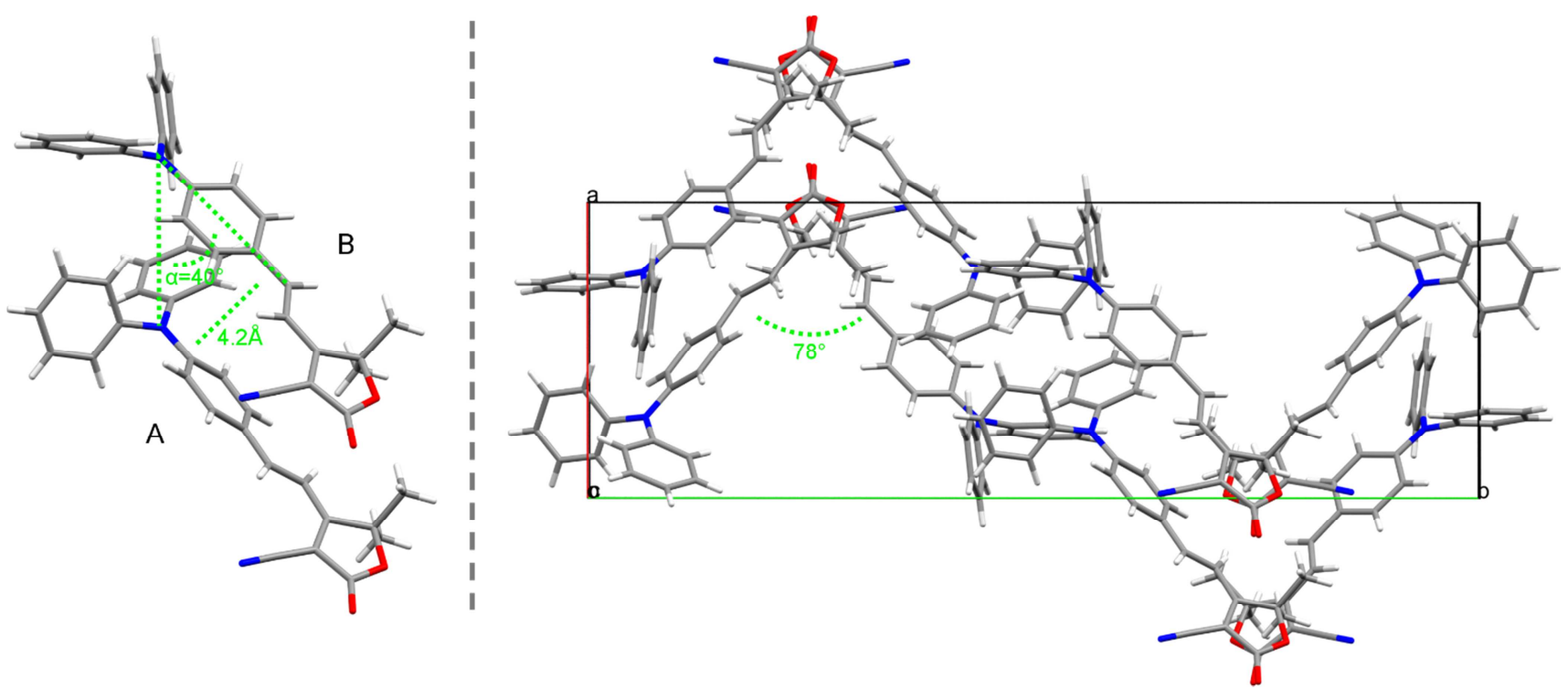

Figure 11. Crystal packing of $\mathbf{1 g}$ viewed along $c$ crystallographic axis showing the two independent molecules A and B (left) and the herringbone pattern (right). Notice the different the $s$-cis conformation of molecule A.

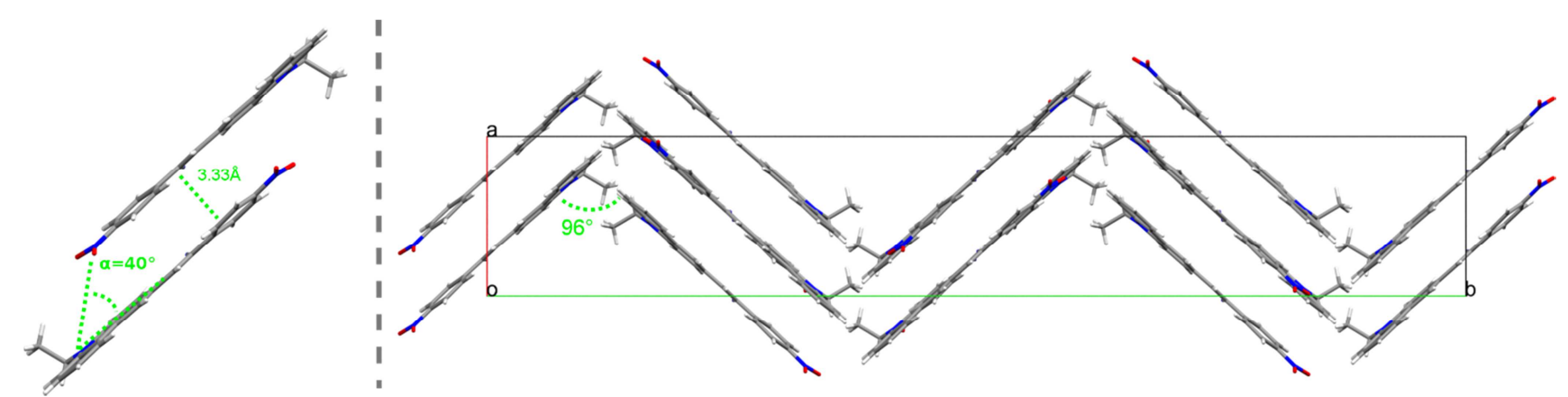

Figure 12. Crystal packing of $\mathbf{2 b}$ viewed along $c$ crystallographic axis showing the herringbone pattern (right) created by repetition of the dimer (left).

Well-ordered herringbone patterns are found in the packing of compounds $\mathbf{1 b}$ (Figure 10), $\mathbf{1 g}$ (Figure 
11-right), and $\mathbf{2 b}$ (Figure 12-right). For compound $\mathbf{1 b}$ the four molecules of the unit cell arrange headto-tail two-by-two forming accentuated vertices $\left(64^{\circ}\right)$. Two head-to-tail dipole are offset relative to one another slipped away both along the main molecular axis and perpendicular to that direction but in the molecular plane, preventing tight packing. 1b is the compound displaying the weakest emission (4\%). For compound 1g, the two crystallographic independent molecules form a dimer (Figure 11-left). The two molecules are aligned in the same direction at roughly $4.2 \AA$ apart but slipped away from $3.95 \AA$ with a slip angle of $48^{\circ}$. Then four dimers arrange tail-to-tail then head-to-head in the unit cell forming a herringbone pattern growing in the $b$ axis with vertices at $78^{\circ}$ (Figure 11-right). The herringbone packing of $\mathbf{2 b}$ is best viewed down crystallographic axis $c$ (Figure 12-right). Obtuse vertices $\left(96.5^{\circ}\right)$ are formed by two broken lines of dimers. In the dimers the dipoles are anti-parallel to one another, lying $3.33 \AA$ apart and are slipped away along the main molecular axis presenting a slip angle of ca $40^{\circ}$ (Figure 12-left).

Finally, compound 1i crystallized with one half disordered molecule of dichloromethane. The four molecules of the unit cell are almost in the same plane, rotated relative to each other by an angle of almost $90^{\circ}$ about an axis passing through the centers (Figure 13) and connected by two $2 \mathrm{H}$-bonds $(2.528 \AA)$ between $\mathrm{C}=\mathrm{O}$ and $\mathrm{C}-\mathrm{H}$ benzothiazole ring of the closest nearby molecule. Dichloromethane molecules are in the cell corner and intercalate in the center. Again, no close packing was observed in that structure explaining the good emission property obtained (19\% at $673 \mathrm{~nm}$ ).

So, characteristic aggregates are present in the crystalline structures of the emissive solids. To further identify the excitons responsible for solid emission, theoretical calculations are therefore now in progress based on the determined crystallographic structures.

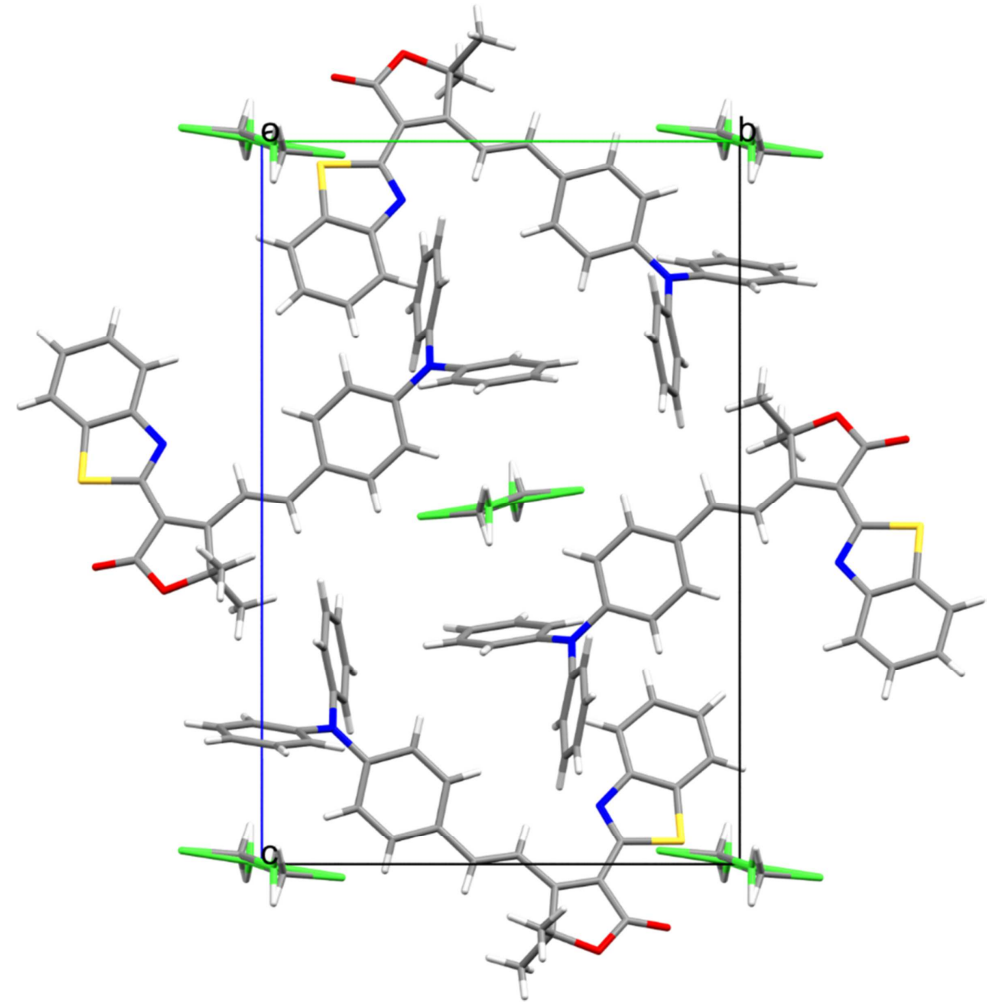

Figure 13. Crystal packing of $\mathbf{1 i}$ viewed along $a$ crystallographic axis. 
681_ Table 3. Crystal data parameters

\begin{tabular}{|c|c|c|c|c|c|c|c|c|c|c|}
\hline & $1 a$ & $1 b$ & 1d & $1 e[a]$ & $1 \mathrm{~g}$ & $1 \mathbf{i}$ & $2 b$ & $2 \mathbf{i}$ & $2 \mathbf{j}$ & $3 f$ \\
\hline Formula & $\mathrm{C}_{28} \mathrm{H}_{19} \mathrm{NO}_{2}$ & $\mathrm{C}_{27} \mathrm{H}_{19} \mathrm{~N}_{3} \mathrm{O}_{22}$ & $2\left(\mathrm{C}_{28} \mathrm{H}_{19} \mathrm{~N}_{3} \mathrm{~S}\right)$ & $\mathrm{C}_{31} \mathrm{H}_{27} \mathrm{~N}_{3} \cdot \mathrm{CH}_{3} \mathrm{CN}$ & $\mathrm{C}_{27} \mathrm{H}_{22} \mathrm{~N}_{2} \mathrm{O}_{2}$ & $2\left(\mathrm{C}_{33} \mathrm{H}_{26} \mathrm{~N}_{2} \mathrm{O}_{2} \mathrm{~S}\right) \cdot \mathrm{CH}_{2} \mathrm{Cl}_{2}$ & $\mathrm{C}_{23} \mathrm{H}_{17} \mathrm{~N}_{3} \mathrm{O}_{2}$ & $\mathrm{C}_{29} \mathrm{H}_{24} \mathrm{~N}_{2} \mathrm{O}_{2} \mathrm{~S}$ & $\mathrm{C}_{28} \mathrm{H}_{25} \mathrm{NO}_{4} \mathrm{~S}$ & $\mathrm{C}_{30} \mathrm{H}_{20} \mathrm{~N}_{4} \mathrm{O}$ \\
\hline Cryst. Syst. & Triclinic & Monoclinic & Triclinic & Monoclinic & Monoclinic & Monoclinic & Orthorhombic & Monoclinic & Monoclinic & Monoclinic \\
\hline Space group & $\begin{array}{c}P \overline{1} \\
\text { (No. 2) }\end{array}$ & $\begin{array}{l}P 2_{1} / c \\
\text { (No. 14) }\end{array}$ & $\begin{array}{l}P \overline{1} \\
\text { (No. 2) }\end{array}$ & $\begin{array}{l}P 2_{1} / c \\
\text { (No. 14) }\end{array}$ & $\begin{array}{l}P 2_{1} / n \\
\text { (No. 14) }\end{array}$ & $\begin{array}{l}P 2_{1} / n \\
\text { (No. 14) }\end{array}$ & $\begin{array}{l}\text { Fdd2 } \\
\text { (No. 43) }\end{array}$ & $\begin{array}{l}P 2_{1} / c \\
\text { (No. 14) }\end{array}$ & $\begin{array}{l}P 2_{1} / c \\
\text { (No. 14) }\end{array}$ & $\begin{array}{l}P 2_{1} \\
\text { (No. 4) }\end{array}$ \\
\hline$a(\AA)$ & $6.8190(10)$ & $11.1416(9)$ & $10.7820(10)$ & $9.173(5)$ & $10.6973(4)$ & $9.407(2)$ & $9.1067(7)$ & $16.006(3)$ & $17.628(3)$ & $10.0378(8)$ \\
\hline$b(\AA)$ & $8.1613(9)$ & $7.5222(7)$ & $14.020(4)$ & $18.158(5)$ & $31.2560(10)$ & $13.981(3)$ & $55.697(5)$ & $18.053(3)$ & $8.2190(10)$ & $7.0649(8)$ \\
\hline$c(\AA)$ & $18.129(2)$ & $25.148(2)$ & $16.248(2)$ & $16.718(5)$ & $13.4698(6)$ & $21.152(4)$ & $14.2490(10)$ & $8.2330(10)$ & $16.809(2)$ & $16.579(2)$ \\
\hline$\alpha\left(^{\circ}\right)$ & $81.279(9)$ & 90 & $73.790(10)$ & 90.0 & 90 & 90 & 90 & 90 & 90 & 90 \\
\hline$\beta\left(^{\circ}\right)$ & $86.530(10)$ & $97.129(8)$ & $81.800(10)$ & $97.576(5)$ & $104.842(4)$ & $92.61(2)$ & 90 & $97.259(3)$ & $101.55(2)$ & $98.017(8)$ \\
\hline$\gamma\left({ }^{\circ}\right)$ & $86.000(10)$ & 90 & $72.340(10)$ & 90.0 & 90 & 90 & 90 & 90 & 90 & 90 \\
\hline$V\left(\AA^{3}\right)$ & $993.5(2)$ & 2091.3(3) & $2242.8(7)$ & $2760(19)$ & $4353.4(3)$ & $2779.0(10)$ & $7227.3(10)$ & $2359.9(7)$ & $2386.0(6)$ & $1164.2(2)$ \\
\hline z & 2 & 4 & 2 & 4 & 8 & 2 & 16 & 4 & 4 & 2 \\
\hline
\end{tabular}




\section{Conclusion}

A library of twenty-two push-pull fluorophores featuring three different electron-donors groups, i.e. 4( $\mathrm{N}, \mathrm{N}$-diphenylamino)phenyl-, 9-ethyl-9H-carbazolyl- or and 4-(9H-carbazol-9-yl)-, and various electron-acceptor groups were synthesized and their optical properties in solution, nanoparticle and crystal-state characterized. This study was supported by crystallographic analyses of the molecular packing in the crystal-state. Typical AIE was demonstrated by straightforward nanoprecipitation procedure involving solvent shifting process. The emission of the nanoparticles was characterized by red-shifted and enhanced emissions compared with the solution. For crystalline powders, intense emissions in the red and even in the far-red were achieved, reaching a remarkable $11 \%$ quantum yield for an emission at $735 \mathrm{~nm}$. Confirming previous observations on push-pull dipolar solid-state emitters, the crystal-state emissions were related to the existence of specific molecular packing in the crystal structures. This study showed that considerable red-shift in the emission was possible by simple modulation of the strength of the electron-acceptor group, the same tendency being observed in nanoparticles and in the crystal-state although significant differences in the emission maxima wavelengths between the two states are noticed. Work has now started in exploiting the interesting crystal-state properties of the most emissive compounds for deep in vivo imaging. In particular, we aim at designing stable aqueous dispersions of bright crystalline fluorescent nanoparticles of defined size and narrow size distribution for which acquaintance with the physico-chemical parameters controlling the precipitation and the evolution of the suspension is required.

\section{Acknowledgements}

This work was funded by Région Rhône-Alpes through a PhD Grant for G E and financial support from Agence Nationale de la Recherche (ANR-11-BS08-0017 ULTRABRIGHT-TRACERS).

\section{Supplementary data}

Supplementary data related to this article include additional spectroscopic and crystallographic data and figures, as well as complete ${ }^{1} \mathrm{H}$ and ${ }^{13} \mathrm{C}$ NMR data for all compounds.

\section{References}

[1] Peyghambarian N, Norwood RA. Organic optoelectronics: Materials and devices for photonic applications, part ii. Optics and Photonics News 2005;16(4):28-33.

[2] Wu H, Ying L, Yang W, Cao Y. Progress and perspective of polymer white light-emitting devices and materials. Chem Soc Rev 2009;38(12):3391-400.

[3] Reisch A, Klymchenko AS. Fluorescent polymer nanoparticles based on dyes: Seeking brighter tools for bioimaging. Small 2016;12(15):1968-92.

[4] Fery-Forgues S. Fluorescent organic nanocrystals and non-doped nanoparticles for biological applications. Nanoscale 2013;5(18):8428-42.

[5] Mei J, Leung NLC, Kwok RTK, Lam JWY, Tang BZ. Aggregation-induced emission: Together we shine, united we soar! Chem Rev 2015;115(21):11718-940.

[6] Qian J, Wang D, He S. Aggregation-induced emission dyes for in vivo functional bioimaging. Aggregation-induced emission: Fundamentals and applications, volumes 1 and 2: John Wiley and Sons Ltd; 2013. p. 209-37.

[7] Frangioni JV. In vivo near-infrared fluorescence imaging. Curr Opin Chem Biol 2003;7(5):626-34.

[8] Hilderbrand SA, Weissleder R. Near-infrared fluorescence: Application to in vivo molecular imaging. Curr Opin Chem Biol 2010;14(1):71-9.

[9] Ntziachristos V. Going deeper than microscopy: The optical imaging frontier in biology. Nat Meth 2010;7(8):603-14.

[10] Zhang X, Bloch S, Akers W, Achilefu S. Near-infrared molecular probes for in vivo imaging. 
Current Protocols in Cytometry 2012:Unit12.27.

[11] Mei J, Hong Y, Lam JWY, Qin A, Tang Y, Tang BZ. Aggregation-induced emission: The whole is more brilliant than the parts. Adv Mater 2014;26(31):5429-79.

[12] Zhao Z, Geng J, Chang Z, Chen S, Deng C, Jiang T, et al. A tetraphenylethene-based red luminophor for an efficient non-doped electroluminescence device and cellular imaging. J Mater Chem 2012;22(22):11018-21.

[13] Zhao Q, Li K, Chen S, Qin A, Ding D, Zhang S, et al. Aggregation-induced red-nir emission organic nanoparticles as effective and photostable fluorescent probes for bioimaging. $\mathrm{J}$ Mater Chem 2012;22(30):15128-35.

[14] Li K, Zhu Z, Cai P, Liu R, Tomczak N, Ding D, et al. Organic dots with aggregation-induced emission (aie dots) characteristics for dual-color cell tracing. Chem Mater 2013;25(21):4181-7.

[15] Ding D, Mao D, Li K, Wang X, Qin W, Liu R, et al. Precise and long-term tracking of adiposederived stem cells and their regenerative capacity via superb bright and stable organic nanodots. ACS Nano 2014;8(12):12620-31.

[16] Jiang T, Qu Y, Li B, Gao Y, Hua J. Tetraphenylethene end-capped [1,2,5]thiadiazolo[3,4c]pyridine with aggregation-induced emission and large two-photon absorption cross-sections. RSC Adv 2015;5(2):1500-6.

[17] Wang X, Morales AR, Urakami T, Zhang L, Bondar MV, Komatsu M, et al. Folate receptortargeted aggregation-enhanced near-ir emitting silica nanoprobe for one-photon in vivo and two-photon ex vivo fluorescence bioimaging. Bioconjugate Chem 2011;22(7):1438-50.

[18] Wang Z, Yan L, Zhang L, Chen Y, Li H, Zhang J, et al. Ultra bright red aie dots for cytoplasm and nuclear imaging. Polym Chem 2014;5(24):7013-20.

[19] Zhao X, Xue P, Wang K, Chen P, Zhang P, Lu R. Aggregation-induced emission of triphenylamine substituted cyanostyrene derivatives. New J Chem 2014;38(3):1045-51.

[20] Hang Y, Yang L, Qu Y, Hua J. A new diketopyrrolopyrrole-based near-infrared (nir) fluorescent biosensor for bsa detection and aie-assisted bioimaging. Tetrahedron Lett 2014;55(51):6998-7001.

[21] Gao Y, Feng G, Jiang T, Goh C, Ng L, Liu B, et al. Biocompatible nanoparticles based on diketopyrrolo-pyrrole (dpp) with aggregation-induced red/nir emission for in vivo two-photon fluorescence imaging. Adv Funct Mater 2015;25(19):2857-66.

[22] Sanz N, Baldeck PL, Nicoud J-F, Le Fur Y, Ibanez A. Polymorphism and luminescence properties of cmons organic crystals: Bulk crystals and nanocrystals confined in gel-glasses. Solid State Sci 2001;3(8):867-75.

[23] Treussart F, Botzung-Appert E, Ha-Duong N-T, Ibanez A, Roch J-F, Pansu R. Second harmonic generation and fluorescence of cmons dye nanocrystals grown in a sol-gel thin film. ChemPhysChem 2003;4(7):757-60.

[24] Chen C-T. Evolution of red organic light-emitting diodes: $\square$ Materials and devices. Chem Mater 2004;16(23):4389-400.

[25] Chiang CL, Wu MF, Dai DC, Wen YS, Wang JK, Chen CT. Red-emitting fluorenes as efficient emitting hosts for non-doped, organic red-light-emitting diodes. Adv Funct Mater 2005;15(2):231-8.

[26] Ishow E, Brosseau A, Clavier G, Nakatani K, Tauc P, Fiorini-Debuisschert C, et al. Multicolor emission of small molecule-based amorphous thin films and nanoparticles with a single excitation wavelength. Chem Mater 2008;20(21):6597-9.

[27] Davis R, Saleesh Kumar NS, Abraham S, Suresh CH, Rath NP, Tamaoki N, et al. Molecular packing and solid-state fluorescence of alkoxy-cyano substituted diphenylbutadienes: $\mathbf{a}$ Structure of the luminescent aggregates. J Phys Chem C 2008;112(6):2137-46.

[28] Ooyama Y, Uwada K, Kumaoka H, Yoshida K. Drastic solid-state fluorescence enhancement behaviour of phenanthro[9,10-d]imidazole-type fluorescent hosts upon inclusion of carboxylic acids. Eur J Org Chem 2009;2009(34):5979-90.

[29] D'Souza DM, Muschelknautz C, Rominger F, Müller TJJ. Unusual solid-state luminescent push-pull indolones: A general one-pot three-component approach. Org Lett 2010;12(15):3364-7.

[30] Ooyama Y, Sugiyama T, Oda Y, Hagiwara Y, Yamaguchi N, Miyazaki E, et al. Synthesis of 
carbazole-type d- $\pi$-a fluorescent dyes possessing solid-state red fluorescence properties. Eur J Org Chem 2012;2012(25):4853-9.

[31] Gupta VD, Tathe AB, Padalkar VS, Umape PG, Sekar N. Red emitting solid state fluorescent triphenylamine dyes: Synthesis, photo-physical property and dft study. Dyes Pigm 2013;97(3):429-39.

[32] Zheng Z, Yu Z, Yang M, Jin F, Zhang Q, Zhou H, et al. Substituent group variations directing the molecular packing, electronic structure, and aggregation-induced emission property of isophorone derivatives. J Org Chem 2013;78(7):3222-34.

[33] Yang M, Xu D, Xi W, Wang L, Zheng J, Huang J, et al. Aggregation-induced fluorescence behavior of triphenylamine-based schiff bases: The combined effect of multiple forces. J Org Chem 2013;78(20):10344-59.

[34] Yang Q-Y, Lehn J-M. Bright white-light emission from a single organic compound in the solid state. Angew Chem Int Ed 2014;53(18):4572-7.

[35] Wang L, Shen Y, Zhu Q, Xu W, Yang M, Zhou H, et al. Systematic study and imaging application of aggregation-induced emission of ester-isophorone derivatives. J Phys Chem C 2014;118(16):8531-40.

[36] Ruanwas P, Boonnak N, Chantrapromma S. Five different colours solid-state fluorescence of azastilbenes: A new push-pull $\pi$-conjugated system. Bull Mater Sci 2015;38(3):791-5.

[37] Zhang Y, Pan J, Zhang C, Wang H, Zhang G, Kong L, et al. High quantum yield both in solution and solid state based on cyclohexyl modified triphenylamine derivatives for picric acid detection. Dyes Pigm 2015;123:257-66.

[38] Lanke SK, Sekar N. Aggregation induced emissive carbazole-based push pull nlophores: Synthesis, photophysical properties and dft studies. Dyes Pigm 2016;124:82-92.

[39] Singh A, Lim C-K, Lee Y-D, Maeng J-h, Lee S, Koh J, et al. Tuning solid-state fluorescence to the near-infrared: A combinatorial approach to discovering molecular nanoprobes for biomedical imaging. ACS Appl Mater Interfaces 2013;5(18):8881-8.

[40] Massin J, Dayoub W, Mulatier J-C, Aronica C, Bretonnière Y, Andraud C. Near-infrared solidstate emitters based on isophorone: Synthesis, crystal structure and spectroscopic properties. Chem Mater 2011;23(3):862-73.

[41] Ipuy M, Liao Y-Y, Jeanneau E, Baldeck PL, Bretonniere Y, Andraud C. Solid state red biphotonic excited emission from small dipolar fluorophores. J Mater Chem C 2016;4(4):766-79.

[42] Cheng Y, Li G, Liu Y, Shi Y, Gao G, Wu D, et al. Unparalleled ease of access to a library of biheteroaryl fluorophores via oxidative cross-coupling reactions: Discovery of photostable nir probe for mitochondria. J Am Chem Soc 2016;138(14):4730-8.

[43] Shao A, Xie Y, Zhu S, Guo Z, Zhu S, Guo J, et al. Far-red and near-ir aie-active fluorescent organic nanoprobes with enhanced tumor-targeting efficacy: Shape-specific effects. Angew Chem Int Ed 2015;54(25):7275-80.

[44] Ju H, Wan Y, Yu W, Liu A, Liu Y, Ren Y, et al. Structure and properties of a novel yellow emitting material for organic light-emitting diodes. Thin Solid Films 2006;515(4):2403-9.

[45] Hou J, Pan Y, Jin J-Y, Wu X, Su Z-M. Isophorone-based analogues with a- $\pi$-d- $\pi$-a structure for red organic light emitting devices. Synth Met 2009;159(5-6):401-5.

[46] Gao Z, Zhang X, Chen Y. Red fluorescence thin film based on a strong push-pull dicyanoisophorone system. Dyes Pigm 2015;113:257-62.

[47] Wang YJ, Shi Y, Wang Z, Zhu Z, Zhao X, Nie H, et al. A red to near-ir fluorogen: Aggregationinduced emission, large stokes shift, high solid efficiency and application in cell-imaging. Chem Eur J 2016;22(28):9784-91.

[48] Gao M, Su H, Li S, Lin Y, Ling X, Qin A, et al. An easily accessible aggregation-induced emission probe for lipid droplet-specific imaging and movement tracking. Chem Commun 2017;53(5):921-4.

[49] Zhang X, Gan X, Yao S, Zhu W, Yu J, Wu Z, et al. Branched triphenylamine-core compounds: Aggregation induced two-photon absorption. RSC Adv 2016;6(65):60022-8.

[50] Qi C, Ma H, Fan H, Yang Z, Cao H, Wei Q, et al. Study of red-emission piezochromic materials 
based on triphenylamine. ChemPlusChem 2016;81(7):637-45.

[51] Otomo A, Aoki I, Miki H, Tazawa H, Yokoyama S. Second-order nonlinear optical compounds with good nonlinear optical properties and heat resistance. National Institute of Information and Communications Technology, Japan; Sumitomo Electric Industries, Ltd.; Kyushu University, National University Corporation . 2011. p. 342pp.

[52] Moreno-Yruela C, Garin J, Orduna J, Franco S, Quintero E, Lopez Navarrete JT, et al. D- $\pi$-a compounds with tunable intramolecular charge transfer achieved by incorporation of butenolide nitriles as acceptor moieties. J Org Chem 2015;80(24):12115-28.

[53] Park CP, Nagle A, Yoon CH, Chen C, Jung KW. Formal aromatic $\mathrm{c}-\mathrm{h}$ insertion for stereoselective isoquinolinone synthesis and studies on mechanistic insights into the $\mathrm{c}-\mathrm{c}$ bond formation. J Org Chem 2009;74(16):6231-6.

[54] Monçalves M, Rampon DdS, Schneider PH, Rodembusch FS, Silveira CdC. Divinyl sulfides/sulfones-based $\mathrm{d}-\pi-\mathrm{a}-\pi-\mathrm{d}$ dyes as efficient non-aromatic bridges for $\pi$-conjugated compounds. Dyes Pigm 2014;102:71-8.

[55] Hauck M, Stolte M, Schönhaber J, Kuball H-G, Müller TJJ. Synthesis, electronic, and electrooptical properties of emissive solvatochromic phenothiazinyl merocyanine dyes. Chem Eur $\mathbf{J}$ 2011;17(36):9984-98.

[56] Pan Q, Fang C, Zhang Z, Qin Z, Li F, Gu Q, et al. Synthesis and characterization of nonlinear optical chromophores containing a-cyan with thermal stability. Opt Mater 2003;22(1):45-9.

[57] Percino MJ, Chapela VM, Cerón M, Castro ME, Soriano-Moro G, Perez-Gutierrez E, et al. Synthesis and characterization of conjugated pyridine-(n-diphenylamino) acrylonitrile derivatives: Photophysical properties. J Mater Sci Res 2012;1(2):181-2.

[58] Pérez-Gutiérrez E, Percino MJ, Chapela VM, Cerón M, Maldonado JL, Ramos-Ortiz G. Synthesis, characterization and photophysical properties of pyridine-carbazole acrylonitrile derivatives. Materials 2011;4(3):562.

[59] Cho MJ, Seo J, Oh HS, Jee H, Kim WJ, Kim KH, et al. Tricyanofuran-based donor-acceptor type chromophores for bulk heterojunction organic solar cells. Sol Energy Mater Sol Cells 2012;98:71-7.

[60] Lakowicz JR. Appendix i. Corrected emission spectra. In: Lakowicz JR, editor. Principles of fluorescence spectroscopy. Boston, MA: Springer US; 2006. p. 873-82.

[61] Briers D, Picard I, Verbiest T, Persoons A, Samyn C. Nonlinear optical active poly(adamantyl methacrylate-methyl vinyl urethane)s functionalised with phenyltetraene-bridged chromophore. Polymer 2004;45(1):19-24.

[62] Collins I. Saturated and unsaturated lactones. J Chem Soc, Perkin Trans 1 1999(11):1377-96.

[63] Rao YS. Recent advances in the chemistry of unsaturated lactones. Chem Rev 1976;76(5):62594.

[64] Zeng L, Ye Q, Oberlies NH, Shi G, Gu Z-M, He K, et al. Recent advances in annonaceous acetogenins. Nat Prod Rep 1996;13(4):275-306.

[65] Tanzila MU, Vladimir VV. Advances in the synthesis of natural butano- and butenolides. Russ Chem Rev 2009;78(4):337.

[66] Bassetti M, D'Annibale A. Formation of five- and six-membered a, $\mathbf{0}$-unsaturated lactones through ring-closing metathesis of functionalized acrylates. Applications to synthesis of natural products. Curr Org Chem 2013;17(22):2654-77.

[67] Avetisyan AA, Dangyan MT. The chemistry of $\mathbf{0}$ ou -butenolides. Russ Chem Rev 1977;46(7):643.

[68] Avetisyan AA, Mangasaryan TA, Melikyan GS, Dangyan MT, Matsoyan SG. Unsaturated lactones. Ii. Synthesis of unsaturated a-lactones by condensation of $\mathbf{0}$-ketoalcohols with acetoacetic and cyanoacetic esters. Zh Org Khim 1971;7(5):962-4.

[69] Avetisyan AA, Tatevosyan GE, Mangasaryan TA, Matsoyan SG, Dangyan MT. Unsaturated lactones. I. Synthesis of substituted unsaturated a-lactones by condensing tertiary $\mathbf{0}$-keto alcohols with malonic ester. Zh Org Khim 1970;6(5):962-4.

[70] Melikyan GS, Avetisyan AA, Halgas J. Benzothiazole compounds. Xliii. Synthesis of benzazoles 
with heterocyclic substituents and their condensation reactions with aldehydes. Chem Pap 1992;46(2):109-12.

[71] Avetisyan KS, Galstyan LK. Synthesis of 2-(2,5-dihydrofuran-3-yl)-2-oxoethyl carboxylates. Russ J Org Chem 2013;49(6):936-9.

[72] Hakobyan RM, Hayotsyan SS, Melikyan GS. Cyclocondensation of 3-acetylfuran-2(5h)-ones with benzylidenemalononitrile. Synthesis of 3-(5-amino-4,6-dicyanobiphenyl-3-yl)furan-2(5h)-ones. Russ J Org Chem 2015;51(12):1809-12.

[73] An B-K, Kwon S-K, Jung S-D, Park SY. Enhanced emission and its switching in fluorescent organic nanoparticles. J Am Chem Soc 2002;124(48):14410-5.

[74] An B-K, Gierschner J, Park SY. a-conjugated cyanostilbene derivatives: A unique self-assembly motif for molecular nanostructures with enhanced emission and transport. Acc Chem Res 2012;45(4):544-54.

[75] Oelkrug D, Tompert A, Egelhaaf H-J, Hanack M, Steinhuber E, Hohloch M, et al. Towards highly luminescent phenylene vinylene films. Synth Met 1996;83(3):231-7.

[76] Oelkrug D, Tompert A, Gierschner J, Egelhaaf H-J, Hanack M, Hohloch M, et al. Tuning of fluorescence in films and nanoparticles of oligophenylenevinylenes. $J$ Phys Chem B 1998;102(11):1902-7.

[77] Font-Sanchis E, Galian RE, Cespedes-Guirao FJ, Sastre-Santos A, Domingo LR, FernandezLazaro F, et al. Alkoxy-styryl dcdhf fluorophores. PCCP 2010;12(28):7768-71.

[78] Mettra B, Appaix F, Olesiak-Banska J, Le Bahers T, Leung A, Matczyszyn K, et al. A fluorescent polymer probe with high selectivity toward vascular endothelial cells for and beyond noninvasive twophoton intravital imaging of brain vasculature. ACS Appl Mater Interfaces 2016;8(27):17047-59.

[79] Li Y, Li F, Zhang H, Xie Z, Xie W, Xu H, et al. Tight intermolecular packing through supramolecular interactions in crystals of cyano substituted oligo(para-phenylene vinylene): A key factor for aggregation-induced emission. Chem Commun 2007(3):231-3.

[80] Kwon OP, Ruiz B, Choubey A, Mutter L, Schneider A, Jazbinsek M, et al. Organic nonlinear optical crystals based on configurationally locked polyene for melt growth. Chem Mater 2006;18(17):4049-54.

[81] Gainsford GJ, Ashraf M, Kay AJ. 2-\{3-cyano-4-[2-(4-diethylamino-2-hydroxyphenyl)ethenyl]5,5-dimethyl-2,5-dihydrofuran-2-ylidene \}malononitrile acetone 0.25-solvate. Acta Crystallogr Sect Sect E: Struct Rep Online 2012;68(10):o2991-o2.

[82] Li S, Li M, Qin J, Tong M, Chen X, Liu T, et al. Synthesis, crystal structures and nonlinear optical properties of three tcf-based chromophores. CrystEngComm 2009;11(4):589-96.

[83] Khodorkovsky V, Mazor RA, Ellern A. 2-(p-diethylaminobenzylidene)-1,3-indandione. Acta Crystallogr Sect C: Cryst Struct Commun 1996;52(11):2878-80.

[84] Würthner F, Kaiser TE, Saha-Möller CR. J-aggregates: From serendipitous discovery to supramolecular engineering of functional dye materials. Angew Chem Int Ed 2011;50(15):3376-410. 